6-8-2021

\title{
How to Apply Multiple Imputation in Propensity Score Matching with Partially Observed Confounders: A Simulation Study and Practical Recommendations
}

\author{
Albee Ling \\ Stanford University, yling@stanford.edu \\ Maria Montez-Rath \\ Stanford University \\ Maya Mathur \\ Stanford University \\ Kris Kapphahn \\ Stanford University \\ Manisha Desai \\ Stanford University
}

Follow this and additional works at: https://digitalcommons.wayne.edu/jmasm

Part of the Applied Statistics Commons, Social and Behavioral Sciences Commons, and the Statistical Theory Commons

\section{Recommended Citation}

Ling, A., Montez-Rath, M., Mathur, M., Kapphahn, K., \& Desai, M. (2020). How to Apply Multiple Imputation in Propensity Score Matching with Partially Observed Confounders: A Simulation Study and Practical Recommendations. Journal of Modern Applied Statistical Methods, 19(1), eP3439. https://doi.org/ $10.22237 /$ jmasm/1608552120

This Regular Article is brought to you for free and open access by the Open Access Journals at DigitalCommons@WayneState. It has been accepted for inclusion in Journal of Modern Applied Statistical Methods by an authorized editor of DigitalCommons@WayneState. 


\section{How to Apply Multiple Imputation in Propensity Score Matching with Partially Observed Confounders: A Simulation Study and Practical Recommendations}

\section{Cover Page Footnote}

This work was supported by a Sanofi iDEA Award. We are particularly grateful for the excellent insights and guidance on this research provided by the Sanofi research team members: Robert LoCasale, Karen Chandross, Liz Zhou and Cliona Molony. We also want to thank Joseph Rigdon, Ariadna Garcia, and Christopher Gardner for providing us the DIETFITS trial data as our real data analysis example. 


\section{How to Apply Multiple Imputation in Propensity Score Matching with Partially Observed Confounders: A Simulation Study and Practical Recommendations}

\author{
Albee Ling \\ Stanford University \\ Stanford, CA
}

\author{
Maria Montez-Rath \\ Stanford University \\ Stanford, CA
}

\author{
Maya Mathur \\ Stanford University \\ Stanford, CA
}

$\begin{array}{ll}\text { Kris Kapphahn } & \text { Manisha Desai } \\ \text { Stanford University } & \text { Stanford University } \\ \text { Stanford, CA } & \text { Stanford, CA }\end{array}$

Propensity score matching (PSM) has been widely used to mitigate confounding in observational studies, although complications arise when the covariates used to estimate the PS are only partially observed. Multiple imputation (MI) is a potential solution for handling missing covariates in the estimation of the PS. However, it is not clear how to best apply MI strategies in the context of PSM. We conducted a simulation study to compare the performances of popular non-MI missing data methods and various MI-based strategies under different missing data mechanisms. We found that commonly applied missing data methods resulted in biased and inefficient estimates, and we observed large variation in performance across MI-based strategies. Based on our findings, we recommend 1) estimating the PS after applying MI to impute missing confounders; 2) conducting PSM within each imputed dataset followed by averaging the treatment effects to arrive at one summarized finding; 3) a bootstrapped-based variance to account for uncertainty of PS estimation, matching, and imputation; and 4) inclusion of key auxiliary variables in the imputation model.

Keywords: propensity score matching, missing covariates, multiple imputation, confounders, observational studies, causal inference

doi: 10.22237/jmasm/1608552120 | Accepted: Oct. 14, 2020; Published: June 8, 2021.

Correspondence: Albee Ling, yling@stanford.edu 


\section{Introduction}

Randomized clinical trials serve as the gold standard for providing strong evidence for the effects of new and existing treatments for disease and disease prevention (Concato, Shah, \& Horwitz, 2000). For numerous reasons including ethical and financial costs, however, such trials are not always feasible to conduct. Alternatively, observational studies have a long history of providing evidence for comparative effectiveness of treatments and interventions, and can also serve as justification for conducting a definitive randomized clinical trial (Fleurence, Naci, \& Jansen, 2010; Holcomb et al., 2013; Lauer \& Collins, 2010). The presence of confounding, however, can threaten the ability of an observational study to draw causal inference (Cochran \& Rubin, 1973). Methods based on the propensity score (PS), defined as the conditional probability of being assigned a particular treatment given the subject's observed baseline covariates, can be used to mitigate such issues (D’Agostino, 1998; Goodman, Schneeweiss, \& Baiocchi, 2017; Rosenbaum, 1987; Rosenbaum \& Rubin, 1983). While the true PS is not typically known, it can be estimated using a variety of techniques (Austin, 2011a). Traditionally, PS-based methods include matching, inverse probability of treatment weighting (IPTW), stratification or subclassification, and covariate adjustment (Austin, 2011a; D’Agostino, 1998; Lunceford \& Davidian, 2004; Rosenbaum \& Rubin, 1984). Matching approaches are typically used to estimate the average treatment effect in the treated (ATT), whereas weighting, stratification, and covariate adjustment are more commonly used to estimate the population average treatment effect (ATE). More recently, several new PS-based approaches have emerged (F. Li, Morgan, \& Zaslavsky, 2018; L. Li \& Greene, 2013). Although they often produce results similar to those of regression adjustment (Vable et al., 2019), PS-based methods have several notable advantages. They allow separation of study design and analysis, enable assessment of overlap in covariates and their balance after adjustment, and are especially useful when the outcomes are rare and treatment is common (Austin, 2011a). PS matching (PSM) - where individuals with comparable PSs and discordant exposures are matched to achieve balance in covariates across the comparator groups of interest - is one of the more common tools used among the PS-based techniques and, thus the primary focus of the study presented here.

Under the potential outcomes framework (Imbens, 2004; Rubin, 1974), PSM

produces unbiased estimates of the ATT with the assumptions of strongly ignorable treatment assignment (SITA) and Stable Unit Treatment Value Assumption (SUTVA) (Rosenbaum \& Rubin, 1983). SITA requires 1) the exposure to be independent of potential outcomes given a set of covariates (unconfoundedness) 2) 


\section{MULTIPLE IMPUTATION IN PROPENSITY SCORE MATCHING}

the probability of receiving each treatment conditional on any set of covariates to be strictly between zero and one (positivity) (Rubin, 1986). SUTVA states that the outcome of a subject is not affected by the treatment assignment of other subjects (Rubin, 1986). Once these assumptions are met, researchers need to make a series of decisions involving matching methods including but not limited to: greedy or optimal matching, matching with or without replacement, one-to-one or many-toone matching, and the use of calipers (Stuart, 2010). For example, introducing a caliper to calibrate the required distance between matched observations can aid the quality of matches by discarding units outside the area of common support, further reducing the bias; however, caliper matching can also induce potential bias and reduce efficiency as a result of incomplete matching (Crump, Hotz, Imbens, \& Mitnik, 2009; Rosenbaum \& Rubin, 1985a, 1985b; Stuart, 2010). In this paper, we focus on 1:1 nearest neighbor matching, a commonly used greedy matching algorithm, without replacement with caliper.

Once balance of covariates has been achieved in the matched samples, an analysis can be conducted to estimate the treatment effect and its variance. In contrast to a simple comparison between the treatment groups within the matched samples, a regression-based treatment effect estimator removes residual imbalance in covariates between treatment groups by adjusting for confounders in the model after matching (Ho, Imai, King, \& Stuart, 2007; Schafer \& Kang, 2008; Stuart, 2010; Wan, 2019). The variance estimation of the treatment effect in the context of PSM is not straightforward and remains controversial despite the large body of literature devoting attention to this issue (Abadie \& Imbens, 2006, 2008, 2016; Abadie \& Spiess, 2016; Austin, 2008; Austin \& Small, 2014; Hill \& Reiter, 2006; Ho et al., 2007; Lechner, 2002; Schafer \& Kang, 2008; Stuart, 2008, 2010). In addition to the uncertainty in the treatment effect estimation, researchers disagree on how to account for uncertainty in the PS estimation (Abadie \& Imbens, 2016; Stuart, 2010) or in the matching process (Abadie \& Spiess, 2016; Hill \& Reiter, 2006; Lechner, 2002), if at all. Based on the current literature, we considered two variance estimators as relevant choices: a robust cluster variance estimator (Abadie $\&$ Spiess, 2016) to account for the clustering induced by matched observations as well as a bootstrapped-based estimator (Austin \& Small, 2014; Efron \& Tibshirani, 1994) as it takes into account uncertainties in both the PS estimation and the matching process.

The statistical validity of PSM is threatened in the presence of missing data (D’Agostino Jr, 2004; D’Agostino Jr \& Rubin, 2000; Ibrahim, Lipsitz, \& Chen, 1999; Rosenbaum \& Rubin, 1984). For example, if systematic missingness exists among measured confounders, the estimated ATT may be biased. The most 
common approaches to handling partially observed confounders in PSM include complete-case analyses (CC), complete-variable analysis (CVA), and single imputation methods (Choi, Dekkers, \& le Cessie, 2019). In the former, subjects missing at least one confounder are excluded from the analysis (Malla et al., 2018; White \& Carlin, 2010). Importantly, CC produces unbiased estimates when data are missing completely at random (MCAR), i.e. missingness is not related to either observed or unobserved data. In contrast to CC, CVA is a different method that involves excluding confounders with missingness from the analysis. Single imputation methods have also been applied in this context, although less frequently than CC and CVA (Choi et al., 2019). Multiple imputation (MI) is a reasonably flexible method for handling missing data with good statistical properties that leads to unbiased and efficient estimators of parameters of interest when the data are missing at random (MAR), i.e., when the missingness is related to observed data only and specifically not unobserved data conditional on the observed (Little \& Rubin, 2014). MI may also be applicable when data are missing not at random (MNAR), i.e., when missingness is related to unobserved variables, although researchers need to explicitly model the missing data mechanisms under MNAR (Collins, Schafer, \& Kam, 2001). The implementation of MI even in the simplest of contexts and particularly in the context of PSM, however, requires that the user makes numerous decisions which can greatly impact the results (Van Buuren, 2018). Among the two modelling approaches of MI, our study focuses on fully conditional specification instead of joint modeling for its flexibility to accommodate multiple data types and its increase in application (Azur, Stuart, Frangakis, \& Leaf, 2011).

As alluded to above, MI presents unique issues in the context of PSM. To incorporate the PS when using MI, one has to (1) estimate the PS and (2) integrate the PS into the analysis to obtain the treatment effect. There are multiple options for applying MI in the estimation step. Specifically, it is not clear whether one should impute the confounders first and then estimate the PS, referred to as a passive approach (Van Buuren, 2018), or whether one should impute the PS as if it were any other variable, referred to as an active approach (Von Hippel, 2009). The question of imputing in the presence of derived variables is not new and has been discussed in previous contexts, including for imputing interaction terms and higherorder terms (Desai, Mitani, Bryson, \& Robinson, 2016; Mitani, Kurian, Das, \& Desai, 2015; S. R. Seaman, Bartlett, \& White, 2012; Von Hippel, 2009; White, Royston, \& Wood, 2011). However, the approach utilized in the context of PSM has been limited (B. B. L. P. de Vries \& Groenwold, 2017; Granger, Sergeant, \& Lunt, 2019; Hill, 2004; Mitra \& Reiter, 2016). Active approaches have been 


\section{MULTIPLE IMPUTATION IN PROPENSITY SCORE MATCHING}

promoted as bias-reducing because all variables and their interrelationships are considered in the imputation process, reflecting principles behind a proper and congenial imputation approach (Meng, 1994; Rubin, 2004; Rubin \& Thomas, 1996; Van Buuren, 2018). In contrast, passive approaches have been supported because they result in internally consistent imputations (where the PS for subjects will perfectly correspond to its estimation as a function of their underlying confounders). Regarding the integration of PS, one can apply PSM within each imputed dataset and then arrive at an overall treatment effect estimate by averaging the effects obtained across imputed datasets (known as within integration). Alternatively, one can average the PSs across the imputed datasets to obtain one PS before estimating treatment effect from PSM (known as across integration) (B. B. L. P. de Vries \& Groenwold, 2017; Granger et al., 2019; Hill, 2004; Leyrat et al., 2019; Mitra \& Reiter, 2016).

We are not the first to consider MI methods when using PSM for causal inference (B. B. L. P. de Vries \& Groenwold, 2017; Granger et al., 2019; Hill, 2004; Mitra \& Reiter, 2016). However, significant gaps in methods remain, as work to date has been limited and has consisted of only one form of passive imputation (where confounders are first imputed without consideration of the PS, which is subsequently estimated) along with within and across integration strategies (B. B. L. P. de Vries \& Groenwold, 2017; Granger et al., 2019; Hill, 2004; Mitra \& Reiter, 2016). We build upon this excellent body of literature by evaluating active imputations and variations of passive imputations that allow the consideration of auxiliary terms in the imputation model. Further, there is no consensus on how to best estimate the uncertainty of the treatment effect within this framework. This paper presents a novel simulation study to comprehensively evaluate MI imputation and integration approaches in the context of PSM for the purpose of causal inference. We detail gaps in the current literature that examined MI for PSM, describe our methods for conducting a simulation study, present our findings, and discuss interpretation of our findings that inform best statistical practice in the final section.

\section{Background}

MI is a simulation-based statistical tool to handle missing data, which involves three main steps. In Step 1, multiple sets of plausible values of the missing variables are generated based on the posterior predictive distribution of observed variables to reflect the uncertainties of the imputation process. In Step 2, analyses are performed within each imputed dataset, before their results are combined with the application 
of Rubin's Rules in Step 3 (Carpenter \& Kenward, 2012). It has been well established in the MI literature that the outcome should always be included in the imputation process when regression parameters are of interest (B. B. L. de Vries \& Groenwold, 2016; Little \& Rubin, 2014; Moons, Donders, Stijnen, \& Harrell Jr, 2006; Sterne et al., 2009; Van Buuren, 2018). In the context of PSM, the various strategies we consider involve Steps 1 and 3, are described below and summarized in the Glossary.

With respect to Step 1, there are two broad categories of MI strategies that have been introduced in the literature for derived variables or variables that are functions of other variables: active (MI-active) and passive (MI-passive) (Figure 1a). Such derived variables include interaction terms, higher order terms, ratios of two variables (e.g. body mass index), and rates of change (Desai et al., 2016; Mitani et al., 2015; S. R. Seaman et al., 2012; Von Hippel, 2009; White et al., 2011). In MI-active, the derived variable is imputed as if it were any other variable (Von Hippel, 2009). The simplest, regular form of MI-active, MI-regActive, involves calculating the derived variable in complete cases and imputing it together with all other missing variables in the imputation process, with no consideration of its known relationship to the variables involved in its derivation. MI-regActive is a proper imputation method, as all the relationships specified in the scientific model are included in the imputation models, i.e. the imputation model is congenial with the scientific model (Meng, 1994; Rubin, 2004; Rubin \& Thomas, 1996; Van Buuren, 2018). Although MI-active is advantageous given its consideration of the entire covariance structure, some argue that it undermines the imputation process by creating internally inconsistent values. This motivated a re-derived version of MI-active where the derived variable is recalculated post-imputation (MIredActive) (Von Hippel, 2009).

In contrast to MI-active approaches, MI-passive approaches maintain the internal consistency between variables used in the derivation and the derived variable itself (Van Buuren, 2018). In this case, the derived term is not to be imputed but derived after imputing the variables involved in the term's construction. The simplest form of MI-passive is MI-derPassive, where all variables involved in the derivation are imputed prior to deriving the term from the imputed data (Von Hippel, 2009). However, because the derived variable is not included in the imputation process, MI-derPassive may introduce bias. Another form of MI-passive, MI-regPassive, was developed to partially address this issue by including the derived variable in the imputation process of those variables that are not involved in its derivation (Royston, 2009). The latter includes auxiliary variables, which can enhance the imputation process but do not provide any useful 


\section{MULTIPLE IMPUTATION IN PROPENSITY SCORE MATCHING}

information for the scientific model (Collins et al., 2001). Examples include variables associated with the pattern of missingness or the missing variable itself (Collins et al., 2001). Previous work in MI for PSM has been limited to MIderPassive. Neither MI-regPassive, which involves an auxiliary variable, fully or partially observed, nor any active approaches (MI-regActive and MI-redActive) have been considered previously for handling missingness in PSM.

PS estimates need to be integrated in the analysis to estimate the treatment effect. There has been considerable work in examining integration methods for $M I-$ derPassive. Specifically, the PS can be estimated and incorporated within each imputed dataset (INT-within) prior to obtaining the treatment effect through summarization in Step 3, or the PS can be averaged across the imputed datasets after completing Step 1 and applied to the original dataset to obtain the treatment effect (INT-across) (B. B. L. P. de Vries \& Groenwold, 2017; Granger et al., 2019; Hill, 2004; Mitra \& Reiter, 2016). An additional variation on the latter has been previously applied in the context of IPTW (INT-across2) and involves averaging both the estimated regression coefficients corresponding to the covariates used to estimate the PS model and the covariates values themselves to arrive at one PS that can be applied to obtain the treatment effect (Leyrat et al., 2019). The rationale is that the PS coefficients are more suitable for combination using Rubin's Rules given their distributional properties than the PSs themselves, which are confined to be between 0 and 1 (Figure $1 \mathrm{~b}$ ). We will comprehensively evaluate the different combinations of MI imputation and integration strategies described.

How to best estimate the variance of the treatment effect in the context of PSM when applying MI is an open research topic (B. B. L. P. de Vries \& Groenwold, 2017; Hill, 2004; Mitra \& Reiter, 2016). In addition to the complications in variance estimation in PSM mentioned above in the absence of missing data, the uncertainty introduced by the MI process needs to be considered. The application of Rubin's Rules in INT-within accomplishes this goal, but it is unclear how to capture this uncertainty when applying INT-across and INT-across 2 (B. B. L. P. de Vries \& Groenwold, 2017; Hill, 2004; Mitra \& Reiter, 2016). Bootstrap methods have been proposed in the context of MI (Brand, van Buuren, le Cessie, \& van den Hout, 2019; Schomaker \& Heumann, 2018) and specifically with respect to PS-based methods (B. B. L. P. de Vries \& Groenwold, 2017; Qu \& Lipkovich, 2009). For example, Austin \& Small evaluated two potential estimators for PSM in the absence of missing data, where the variance was obtained by either resampling matched pairs or the original observations (Austin \& Small, 2014). 


\section{LING ET AL.}

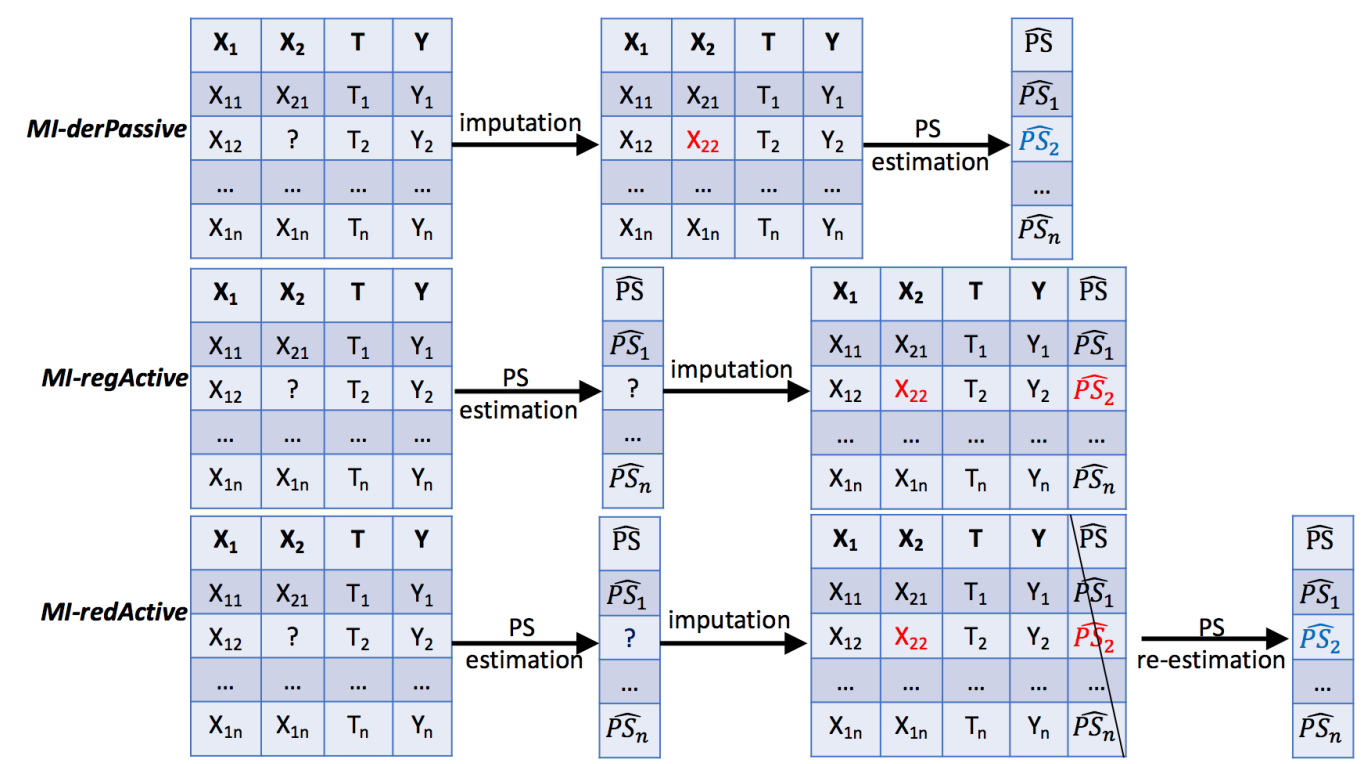

Figure 1a

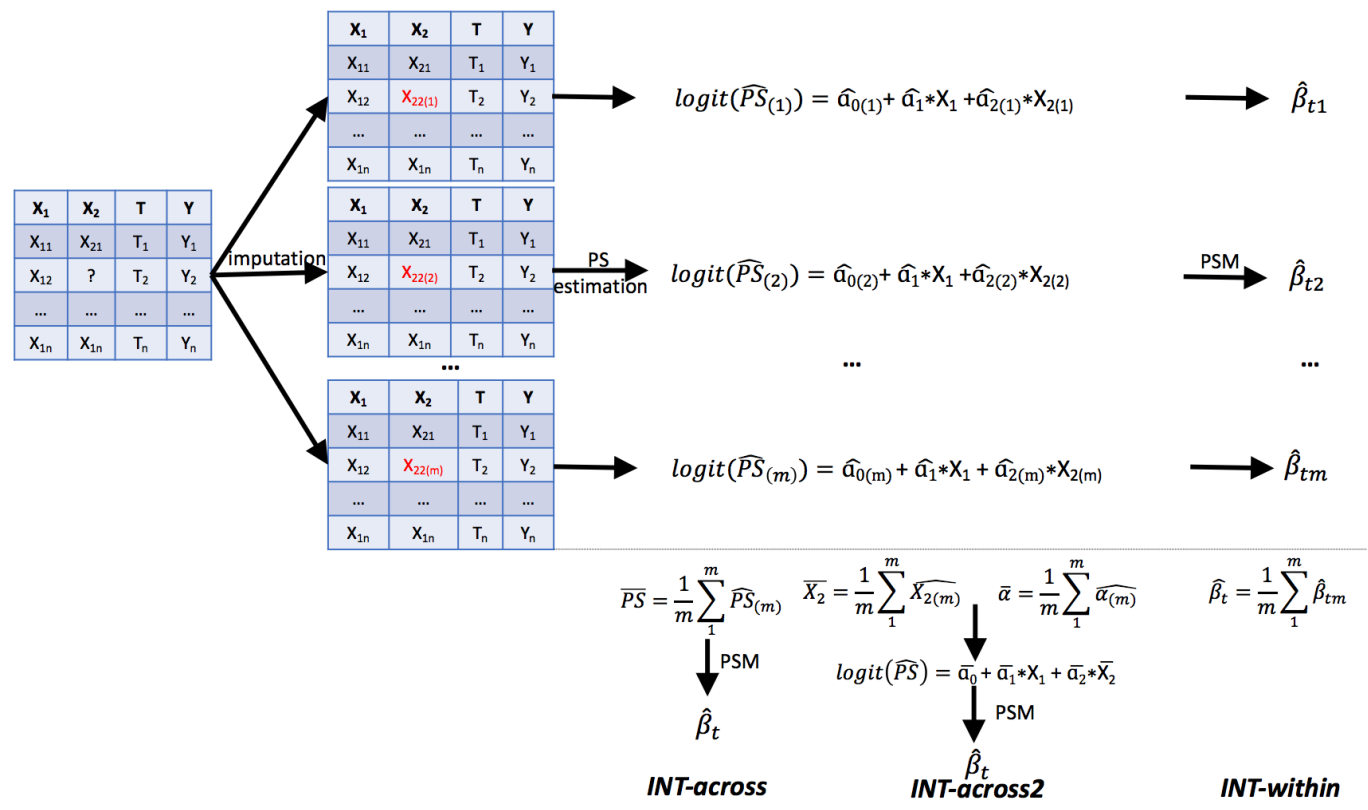

Figure 1b

Figure 1. Figure 1a: Illustration of three imputation strategies demonstrated with one imputed dataset; Figure $1 \mathrm{~b}$ : Illustration of three strategies to integrate the propensity score for estimation of the treatment effect. 


\section{MULTIPLE IMPUTATION IN PROPENSITY SCORE MATCHING}

Although the former performed well relative to the empirical variance, the latter was comparable and enabled extension to the MI context. Schomaker \& Heumann evaluated four bootstrapped-based approaches in the context of MI when PSM was not considered (Schomaker \& Heumann, 2018). One of these approaches, Boot MI, is applicable to the PSM context and overlaps with the ideas described by Austin $\&$ Small. We therefore compare two competing variance estimators in this study to better inform those applying MI in the PSM setting: a bootstrapped-based variance estimator and a robust cluster variance estimator (with Rubin's Rules when applying INT-within) to account for various sources of variation when MI is applied in the context of PSM.

\section{Simulation Study Design}

We conducted an extensive simulation study to assess the performance of various MI-based strategies and commonly applied missing data methods when estimating ATT using PSM. In all scenarios, we included two binary confounders $\left(X_{1}\right.$ and $\left.X_{2}\right)$ of the relationship between treatment and outcome, a binary variable representing the treatment or exposure of interest $(T)$, and a continuous outcome $(Y)$. Two auxiliary variables $\left(Z_{2}\right.$ and $\left.Z_{p s}\right)$ were generated to aid the imputation process. Missing values were present in $X_{2}$ whereas $X_{1}, T$ and $Y$ were always fully observed. For each scenario, 1,000 simulated datasets were generated, each consisting of $\mathrm{n}=2000$ subjects. All data analyses were conducted in $\mathrm{R}$ version 3.5.1 (R Core Team, 2018). MI and PSM were implemented using the mice and Matching packages respectively (Sekhon, 2008; Van Buuren \& Groothuis-Oudshoorn, 2010). The $\mathrm{R}$ code to replicate this study is publicly available in a Github repository at https://github.com/yling2019/psm_mi. Below we provide details on the data generation, missing data mechanisms, missing data methods considered, and metrics for performance evaluation.

\section{Data generation}

To motivate our simulation study, we want to see if treatment variable $T$, an electronic text message intervention, has any effect on the outcome, treatment adherence, where variables $X_{1}$ and $X_{2}$ are confounders such as sex and race. More details of the simulated variables can be found in the rest of the section, whereas more details of the motivating study can be found in a later section, Case Study. 


\section{LING ET AL.}

Confounders. Two binary variables $\mathrm{X}=\left(X_{1}, X_{2}\right)$ that confound the relationship between treatment and outcome were generated, by first creating two variables from a bivariate normal distribution (correlation of 0.5) each with mean 0 and variance 1 , which were then dichotomized at the mean. The resulting distributions of $X_{1}, X_{2}$ are binomial distributions $(p \approx 0.5)$ with correlation around 0.33 .

Treatment indicator. A binary treatment variable $T$ was generated from a binomial distribution such that:

$$
\operatorname{logit}\left(p\left(T=1 \mid X_{1}, X_{2}\right)\right)=\alpha_{0}+\alpha_{1} X_{1}+\alpha_{2} X_{2}
$$

where $\alpha_{1}=\alpha_{2}=2$ so both covariates contributed equally to the treatment assignment. The intercept of the treatment $\alpha_{0}$ was selected such that roughly $30 \%$ of subjects were treated, to reflect real-world datasets where there are often many more control subjects than treated.

Outcome. A continuous outcome variable $Y$ was generated as a linear function of the treatment and both covariates.

$$
Y=\beta_{0}+\beta_{1} X_{1}+\beta_{2} X_{2}+\beta_{t} T+\varepsilon
$$

where $\beta_{1}=\beta_{2}=2$ so both covariates were equally and positively associated with outcome and $\varepsilon \sim N\left(0,10^{2}\right)$. The intercept $\beta_{0}$ was set to zero and the true treatment effect $\beta_{t}$ was set to 2 .

Auxiliary variables. Auxiliary variables $Z_{2}$ and $Z_{p s}$ were generated to be highly correlated with $X_{2}$ and the estimated PS score respectively (based on full observed data without missing data, with correlation of 0.98 ). More specifically, setting $\delta_{02}=1, \delta_{0 p s}=0, \delta_{12}=\delta_{1 p s}=10$, the auxiliary variables were generated as:

$$
Z_{2}=\delta_{02}+\delta_{12} X_{2}+\varepsilon_{2} ; Z_{p s}=\delta_{0 p s}+\delta_{1 p} P \hat{S}+\varepsilon_{p s}
$$

where $\varepsilon_{2} \sim N\left(0,1^{2}\right)$ and $\varepsilon_{p s} \sim N\left(0,1^{2}\right)$ denote errors generated from standard normal distributions independent from all other variables in the data generating models. 


\section{MULTIPLE IMPUTATION IN PROPENSITY SCORE MATCHING}

\section{Missing data mechanisms (MDMs)}

Missingness was always induced in $X_{2}$, whereas $X_{1}$ was fully observed. Missingness occurred under five different mechanisms: MCAR, MAR1, MAR2A, missing not at random, and an MAR2B scenario. Whereas MAR1 represented a simple MAR scenario, MAR2A and MAR2B were more sinister scenarios that captured the complexity of MDM in real-world datasets. In MAR1, MAR2A, and MNAR, missingness was related to treatment and outcome. In MAR2A, missingness was also related to $Z_{2}$, the auxiliary variable associated with $X_{2}$. In MNAR, missingness was also related to $X_{2}$. Let $R_{2}$ be an indicator variable denoting whether $X_{2}$ is missing $\left(R_{2}=1\right)$ or not $\left(R_{2}=0\right)$. We set $Y_{b}$ and $Z_{2 b}$ to be dichotomizations at the median of the outcome variable $Y$ and auxiliary variable $Z_{2}$ respectively. Under each MDM, the intercept $\gamma_{0}$ was selected such that $50 \%$ of the observations were missing. Let $\gamma_{11}=5$, and $\gamma_{00}=1$, and $I$ be an indicator variable. Missingness in $X_{2}$ was induced as follows:

$$
\begin{aligned}
\text { MAR1: } \operatorname{logit}\left(R_{2}=1 \mid \text { complete data }\right) & =\gamma_{0}+\gamma_{11} I\left(t=1, y_{b}=1\right)+\gamma_{00} I\left(t=0, y_{b}=0\right) \\
\text { MAR2A: } \operatorname{logit}\left(R_{2}=1 \mid \text { complete data }\right) & =\gamma_{0}+\gamma_{11} I\left(t=1, y_{b}=1, Z_{2 b}=1\right) \\
& +\gamma_{00} I\left(t=0, y_{b}=0, Z_{2 b}=0\right) \\
\text { MNAR: } \operatorname{logit}\left(R_{2}=1 \mid \text { complete data }\right) & =\gamma_{0}+\gamma_{11}\left(t=1, y_{b}=1, X_{2}=1\right) \\
& +\gamma_{00}\left(t=0, y_{b}=0, X_{2}=0\right)
\end{aligned}
$$

To study the impact of having a partially observed auxiliary variable, we also induced missingness in $X_{2}$ according to a second MAR2 missing mechanism, MAR2B, based on treatment, outcome, and PS. Letting $Z_{p s b}$ be the dichotomizations of $Z_{p s}$, missingness in $X_{2}$ was induced as follow:

$$
\text { MAR2B: } \begin{aligned}
\operatorname{logit}\left(R_{2}=1 \mid \text { complete data }\right) & =\gamma_{0}+\gamma_{11} I\left(t=1, y_{b}=1, Z_{p s b}=1\right) \\
& +\gamma_{00} I\left(t=0, y_{b}=0, Z_{p s b}=0\right)
\end{aligned}
$$

Additionally, we induced missingness in the auxiliary variable, $Z_{p s}$, under three scenarios that assumed MAR2B for $X_{2}$ : aux_MCAR, aux_MAR1, and aux_MAR2. In both aux_MAR1 and aux_MAR2, missingness was related to $T$ and $P S_{b}$, where $P S_{b}$ is the dichotomization at the median of the PS estimated using full data prior to inducing missingness. Let $R_{z}$ be an indicator variable denoting whether $Z_{p s}$ is missing $\left(R_{z}=1\right)$ or not $\left(R_{z}=0\right)$. The intercept term $\varepsilon_{0}$ was selected to ensure $20 \%$ missingness in $Z_{p s}$ and missingness can be expressed as: 


$$
\begin{aligned}
\operatorname{logit}\left(R_{z}=1 \mid \text { complete data }\right) & =\varepsilon_{0}+\varepsilon_{11} I\left(t=1, P S_{b}=1\right)+\varepsilon_{10} I\left(t=1, P S_{b}=0\right) \\
& +\varepsilon_{01} I\left(t=0, P S_{b}=1\right)+\varepsilon_{00} I\left(t=0, P S_{b}=0\right)
\end{aligned}
$$

where

$$
\varepsilon_{11}=5, \varepsilon_{10}=0, \varepsilon_{01}=0, \varepsilon_{00}=5 \text { in aux_MAR1 }
$$

and

$$
\varepsilon_{11}=0, \varepsilon_{10}=5, \varepsilon_{01}=5, \varepsilon_{00}=0 \text { in aux_MAR2. }
$$

\section{Missing data methods}

Common missing data methods. We applied various missing data methods that are widely used in the medical research literature including CC, CVA, mean imputation, and the use of missing data indicators.

Multiple imputation strategies. Figure 1 displays the MI strategies considered for PS estimation and integration. MI-derPassive, MI-regActive, and MI-redActive were applied under MCAR, MAR1, MAR2A, and MNAR conditions with or without auxiliary variable $Z_{2}$ in the imputation model, where $Z_{2}$ had no missing values (Appendix Table A1). Note that since missingness in MAR2A is associated with an auxiliary variable, when the auxiliary variable was not included in the imputation, the MI imputation model is misspecified and the MDM becomes an MNAR scenario. Under the MAR2B MDM, we included an additional partially observed auxiliary variable $Z_{p s}$ in the imputation model when MI-regPassive and $M I$-derPassive were applied. Under this scenario we also examined performance by order of inclusion of the variables in the imputation model (i.e., whether $X_{2}$ was imputed before $Z_{p s}$ or not). Integration approaches considered were INT-within, INT-across, and INT-across 2 . Note that INT-across 2 cannot be combined with MIregActive, as the PS is directly imputed. In MICE, 50 multiply imputed datasets $(m=50)$ (White et al., 2011), five iterations (maxit $=5)$ and default settings for the imputation method (predictive mean matching, or PMM, for continuous variable and logistic regression for binary) were used. The treatment and outcome were included in all imputation models (Van Buuren, 2018). 


\section{MULTIPLE IMPUTATION IN PROPENSITY SCORE MATCHING}

\section{PSM and treatment effect estimation}

We estimated coefficients $\alpha_{1}$ and $\alpha_{2}$ using a correctly specified logistic regression model, $\operatorname{logit}(p(T=1 \mid X))=\alpha_{0}+\alpha_{1} X_{1}+\alpha_{2} X_{2}$. PS scores were estimated as the fitted values of the regression model on the response scale. One-to-one nearest neighbor matching without replacement was applied. Subjects were matched by PS scores with calipers of width that is 0.2 of the standard deviation of the logit of PS (Austin, 2011b; Rosenbaum \& Rubin, 1985a). After matching subjects, the treatment effect or ATT was estimated using standard linear regression methods (Abadie \& Spiess, 2016), by regressing $Y$ on $T$ and confounders $X_{1}$ and $X_{2}$ to obtain the estimate for the beta coefficient representing $T$. Adjustment not done in INT-across strategies because of the presence of multiple sets of $X_{1}$ and $X_{2}$.

\section{Variance estimation}

In the absence of missing data, we used two approaches to estimate the uncertainty of the treatment effect: (1) a robust cluster variance estimate (McCullagh, 2018) that accounts for the matched design and (2) a bootstrapped variance calculated as the standard deviation of treatment effects in 1,000 bootstrapped samples to account for both PS estimation and the matching process. For the latter, the detailed procedure is described as follows:

1. Sample with replacement $n=2000$ rows from the observed dataset $D=(X, T, Y, Z, R)$ to obtain a bootstrapped dataset $D_{\text {boot }}$ which contains missing values;

2. Impute $m$ datasets for $D_{b o o t}$ using the imputation strategy (MIderPassive, MI-regPassive, MI-regActive, or MI-redActive), for $k=1,2, \ldots, m$, denoted as $D_{\text {boot }}(k)$;

3. Apply the integration approach (INT-within, INT-across or INTacross 2 ) to obtain a single effect estimate for $D_{b o o t}$;

4. Repeat steps 1-3 $B$ times to obtain $B$ bootstrap replicates from which treatment effect $\beta_{t b o o t}$ can be estimated for a given bootstrap sample $D_{\text {boot }}$;

5. Calculate bootstrapped standard error as the standard deviation of $B$ treatment effects estimated from each bootstrap sample: $S E_{\text {bootstrap }}=s d\left(\beta_{\text {tboot }}\right)$ for $b=1,2, \ldots, B$ 


\section{LING ET AL.}

When commonly applied missing data methods were considered, the robust cluster variance estimator was used. When MI was applied in the context of PSM, we compared 1) the robust cluster variance estimator and 2) a bootstrapped variance. For the former, when the integration strategy was INT-within, a robust cluster variance was estimated within each of the $\mathrm{m}$ imputed datasets, before application of Rubin's Rules to yield one final variance. For both INT-across and INT-across 2, Rubin's Rules do not apply; instead we obtain only one robust cluster variance.

\section{Sensitivity simulation study}

To test the robustness of our main simulation study, we conducted a second simulation study by reducing coefficients in the treatment and outcome generating models while keeping all other aspects of the simulations the same. To be specific, we set $\alpha_{1}=\alpha_{2}=\log (2)$ and $\beta_{1}=\beta_{2}=1$ to mimic more realistic data examples encountered by applied researchers when data is missing MCAR, MAR1, MAR2A, and MNAR. All statistical analyses were performed in the exact same way as the main simulation study as described above. In order to test the sensitivity of the optimal MI strategy found in the main simulation study, a third set of simulations was conducted by varying the study population size $(n=1000,500,250)$, missing rate $(25 \%, 10 \%)$, or the number of multiply imputed datasets $(m=10)$.

\section{Performance metrics}

After PSM, we examined the percentage of treated subjects matched and the standardized differences of covariates. For mean imputation, standardized differences were calculated in the original full data and the imputed data. For missing indicator variables, standardized differences were calculated in the full data without missingness, as well as its observed and missing part. For INT-within, standardized differences were calculated in 1) each of the imputed datasets, and 2) the full dataset, before averaging over all multiply imputed datasets. For INT-across and INT-across 2 , standardized differences were calculated in 1) the average of $m$ imputed dataset and its observed and imputed parts respectively 2) the full dataset (Leyrat et al., 2019; Moons et al., 2006). For each missing data method, we report on bias, variance, mean squared error (MSE), relative MSE (relative to PSM in the full dataset), and coverage probability summarized over 1,000 simulations per scenario for estimating treatment effect $\beta_{t}$. The robust cluster variance and bootstrapped variance were compared to their corresponding empirical variance for each MI strategy. Coverage was estimated as the proportion of 1,000 simulations 


\section{MULTIPLE IMPUTATION IN PROPENSITY SCORE MATCHING}

such that the interval $\left[\hat{\beta}_{t}-1.96 \times S E, \hat{\beta}_{t}+1.96 \times S E\right]$ contained the true treatment effect of $\beta_{t}=2$ ( $S E$ : robust cluster standard error or bootstrapped standard error). We used the normal theory estimator because the percentile based method did not perform well in simulations by Austin \& Small (Austin \& Small, 2014), and calculating accelerated and bias-corrected confidence intervals (BCa) (Efron \& Tibshirani, 1994) proved too computationally intensive. Monte Carlo standard errors were calculated for bias, empirical standard error, MSE, and coverage (Morris, White, \& Crowther, 2019). Reference metrics for missing data methods were based on applying PSM to the full data (PSM_full).

\section{Results}

We first quantified the confounding effects introduced by our data generation by regressing outcome on treatment only in the full data set without missingness (data not shown in tables). A large bias was present (12.23), indicating a strong confounding effect. Next. we compared the resulting bias and standard error from two methods that adjusted for confounding: (1) fitting the true data generating model or regression adjustment, where both confounders were included as covariates in the regression model and (2) applying PSM to the full data (PSM_full). Both methods yielded unbiased treatment effect estimates (bias $=-0.006$ in both cases). PSM yielded a higher standard error as expected due to discarding unmatched samples $(0.313$ using regression in the full dataset and 0.380 using PSM_full). Coverage reached the nominal level of $95 \%$ using both methods. These results matched well with their corresponding empirical standard error (0.306 and 0.376 respectively). In PSM, the robust cluster standard error and bootstrapped estimators were comparable ( 0.380 in both cases) and close to the empirical (0.376). MSE in PSM_full was 0.141, which was used as the denominator for calculating all rMSEs later.

\section{Commonly applied missing data methods}

Of the commonly applied approaches, $\mathrm{CC}$ had the most favorable MSE relative to that the of PSM full (rMSE ranged 1.857 to 48.658 in various MDMs, Appendix Table A2). CC produced biased treatment estimates (bias $=-2.489,-0.815$, and -1.084 in MAR1, MAR2A, and MNAR respectively) and less efficient estimates relative to PSM_full (robust standard error $=0.537,0.838,0.682,0.682$ in MCAR, MAR1, MAR2A and MNAR respectively vs 0.380 in PSM_full). CVA, mean imputation, and the use of missing indicators yielded greater bias relative to $\mathrm{CC}$ 
(5.058 to 5.059 for CVA; 2.985 to 5.759 for mean imputation; and 2.973 to 5.534 for missing indicator), although their robust cluster variances were smaller than that of CC. Comparisons of statistical properties obtained when not adjusting for $X_{1}$ and $X_{2}$ were similar.

\section{Variance estimation in MI-based strategies}

While the robust cluster variance estimator and the bootstrapped-based variance estimator were comparable in the absence of missing data, differences were observed in the presence of missingness and when MI was applied. Specifically, the robust cluster variance estimator consistently underestimated the empirical variance in INT-across integration strategy. Among the INT-across approaches, the variance ratios (robust/empirical) were much smaller than 1 and only exceeded 0.8 when an auxiliary variable was included (Figure 2). The worst performance for the robust estimator was observed in MI-regActive INT-across approaches, where the variance ratios can be lower than 0.1 . In contrast, the variance was consistently overestimated in INT-within approaches, where the variance ratio surpassed 5 under $M I$-regActive and MI-redActive approaches, especially when an auxiliary term was used. The ratio of the robust estimator for the variance relative to the empirical under INT-across 2 was close to 1 across all MI methods and MDMs. On the other hand, the bootstrapped-based variance was more comparable to the empirical variance across all MI integration strategies; the ratio of bootstrapped variance to the empirical ranged from 0.675 to 1.875 , with mean 1.01 . We did not observe any trend specific to imputation methods, integration methods, or the inclusion of auxiliary variable in the imputation model. All subsequent results were therefore calculated using the bootstrapped-based variance estimator.

\section{Comparing various $\mathrm{MI}$ strategies}

For simplicity, performance of MI strategies under MAR1 is highlighted here (when auxiliary variable was not included in the imputation model). For the majority of the MI strategies, balance was achieved such that the absolute standardized difference in $X_{1}$ and $X_{2}$ between treated and controls based on the imputed dataset was below 0.1 with the exception of MI-regActive (Appendix Table A3). When considering both bias and efficiency, MI-derPassive approaches achieved the lowest rMSE, followed by MI-redActive and MI-regActive (Table 1 and Figure 3). Among the three integration strategies under MI-derPassive approaches, INT-within, INT-across, INT-across 2 were ranked from the lowest to 


\section{MULTIPLE IMPUTATION IN PROPENSITY SCORE MATCHING}
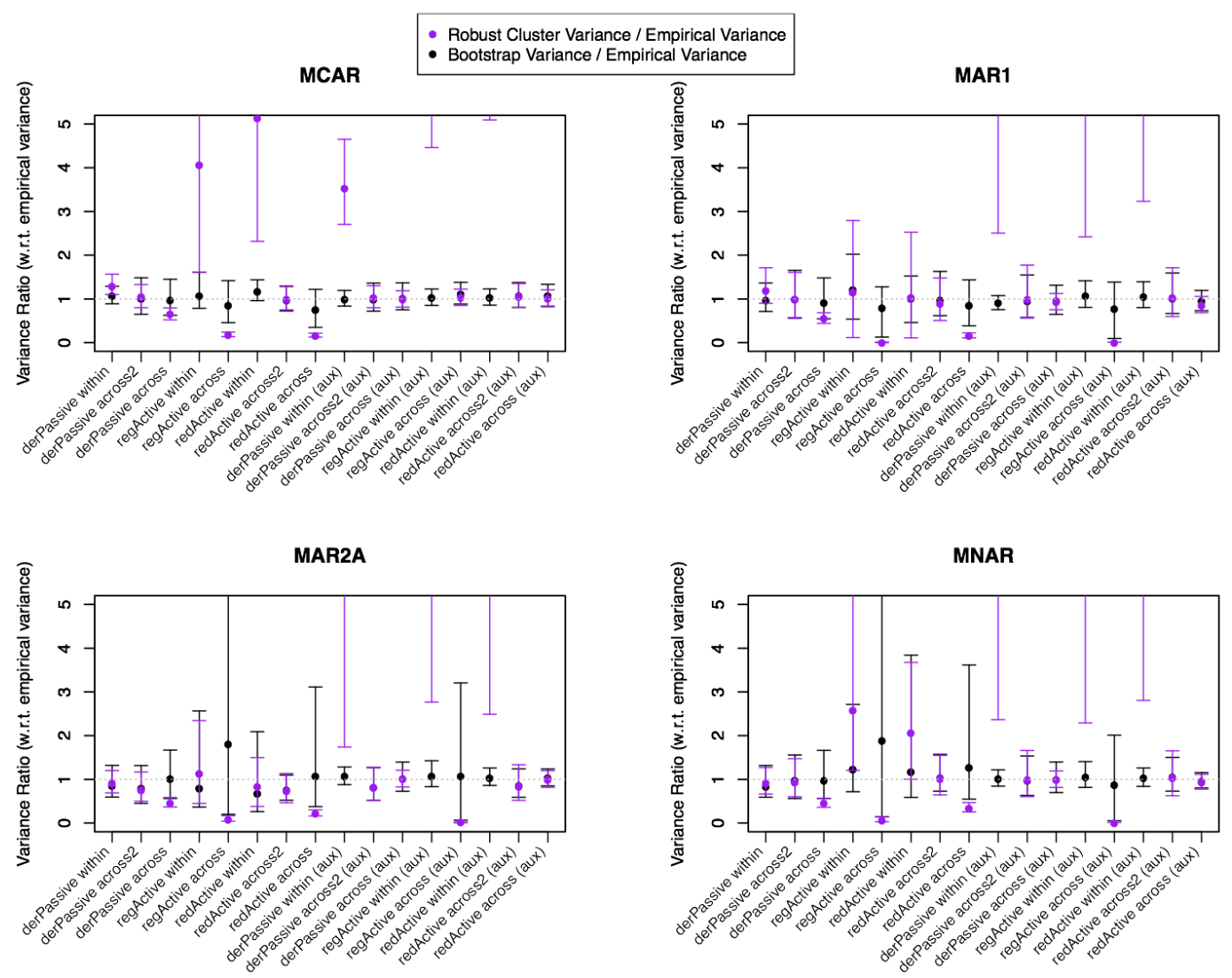

Figure 2. Distribution (mean, 2.5th and 97.5th quantile across 1,000 simulations) of the ratio of robust cluster and bootstrap variances with respect to empirical variance by multiple imputation estimation and integration strategies for propensity score matching. Confidence intervals are trimmed at the value of 5 .

the highest with respect to rMSE, which was largely driven by the bias (Table 1). Similar trend in MI integration strategies was observed for MI-regActive, where INT-within also outperformed INT-across. In contrast, the performance of various integration strategies varied under MI-redActive, where MI-redActive INT-within was the worst performer $(\mathrm{rMSE}=60.454$, bias $=2.557)$ and MI-redActive INTacross was the best performer $(\mathrm{rMSE}=8.784$, bias $=0.465)($ Table 1$)$.

\section{The impact of auxiliary terms}

Under MAR1, when a fully observed auxiliary term, $Z_{2}$, was included in the imputation model, the statistical properties of most MI strategies were comparable 
Table 1. Main simulation results: bias, standard error, mean squared error (MSE), relative mean squared error $(\mathrm{rMSE})^{*}$, and coverage results of various multiple imputation strategies under MAR1 (Monte Carlo standard errors in parentheses).

\begin{tabular}{|c|c|c|c|c|c|c|c|}
\hline \multicolumn{2}{|c|}{ MI Strategy } & \multirow[b]{2}{*}{ Bias } & \multicolumn{2}{|c|}{ Standard Error } & \multirow[b]{2}{*}{ MSE } & \multirow[b]{2}{*}{ rMSE* $^{*}$} & \multirow[b]{2}{*}{ Coverage } \\
\hline Imputation & Integration & & Empirical & Bootstrap & & & \\
\hline \multicolumn{8}{|c|}{ Auxiliary variable not included in imputation model } \\
\hline \multirow{3}{*}{ MI-derPassive } & INT-within & $0.038(0.014)$ & $0.454(0.01)$ & $0.446(0.001)$ & $0.207(0.011)$ & 1.466 & $1(0)$ \\
\hline & INT-across & $-0.176(0.016)$ & $0.497(0.011)$ & $0.472(0.002)$ & $0.278(0.012)$ & 1.969 & $1(0)$ \\
\hline & INT-across2 & $-2.859(0.024)$ & $0.770(0.017)$ & $0.757(0.003)$ & $8.764(0.14)$ & 62.083 & $0(0)$ \\
\hline \multirow{2}{*}{ MI-regActive } & INT-within & $2.471(0.043)$ & $1.345(0.03)$ & $1.463(0.007)$ & $7.913(0.174)$ & 56.055 & $0.823(0.012)$ \\
\hline & INT-across & $5.275(0.173)$ & $5.473(0.122)$ & $4.719(0.033)$ & $57.746(2.605)$ & 409.068 & $0.915(0.009)$ \\
\hline \multirow{3}{*}{ MI-redActive } & INT-within & $2.557(0.045)$ & $1.414(0.032)$ & $1.405(0.006)$ & $8.534(0.183)$ & 60.454 & $0.719(0.014)$ \\
\hline & INT-across & $0.465(0.032)$ & $1.013(0.023)$ & $0.926(0.005)$ & $1.240(0.076)$ & 8.784 & $1(0)$ \\
\hline & INT-across2 & $-2.633(0.025)$ & $0.792(0.018)$ & $0.772(0.003)$ & $7.558(0.132)$ & 53.54 & $0(0)$ \\
\hline \multicolumn{8}{|c|}{ Auxiliary variable included in imputation model } \\
\hline \multirow{3}{*}{ MI-derPassive } & INT-within & $0.319(0.012)$ & $0.395(0.009)$ & $0.376(0.001)$ & $0.258(0.01)$ & 1.828 & $1(0)$ \\
\hline & INT-across & $0.067(0.013)$ & $0.398(0.009)$ & $0.385(0.001)$ & $0.163(0.008)$ & 1.155 & $1(0)$ \\
\hline & INT-across2 & $-2.904(0.024)$ & $0.771(0.017)$ & $0.744(0.003)$ & $9.028(0.141)$ & 63.954 & $0(0)$ \\
\hline \multirow{2}{*}{ MI-regActive } & INT-within & $0.502(0.014)$ & $0.449(0.01)$ & $0.465(0.001)$ & $0.453(0.022)$ & 3.209 & $1(0)$ \\
\hline & INT-across & $4.096(0.176)$ & $5.572(0.125)$ & $4.702(0.040)$ & $47.793(2.478)$ & 338.561 & $0.948(0.007)$ \\
\hline \multirow{3}{*}{ Ml-redActive } & INT-within & $0.461(0.013)$ & $0.409(0.009)$ & $0.421(0.001)$ & $0.380(0.016)$ & 2.692 & $1(0)$ \\
\hline & INT-across & $0.059(0.013)$ & $0.418(0.009)$ & $0.406(0.001)$ & $0.178(0.008)$ & 1.261 & $1(0)$ \\
\hline & INT-across2 & $-2.860(0.024)$ & $0.770(0.017)$ & $0.768(0.003)$ & $8.773(0.14)$ & 62.147 & $0(0)$ \\
\hline
\end{tabular}

* $r M S E$ : the ratio of MSE calculated from each missing data method over MSE obtained from propensity score matched results in the absence of missing data.

to those when auxiliary variable was not in the imputation model (Table 1 and Figure 3). Large improvement in performance was observed for MI-regActive INTwithin, MI-redActive INT-within, and MI-redActive INT-across (Table 1). Under MAR2A, we observed an improvement of performance across a wider range of MIstrategies: MI-derPassive/MI-redActive INT-within/INT-across and MI-regActive INT-within (Appendix Table A4 and Figure 3). For example, the rMSE for MIderPassive INT-within in the absence and presence of auxiliary variable is 17.341 and 1.672 respectively (Appendix Table A4). The auxiliary term improved efficiency for most MI strategies except for MI-regActive INT-across. The auxiliary term improved the absolute bias for most MI strategies except for MI-derPassive INT-across 2 and MI-redActive INT-across2. MNAR results were similar to MAR2A, and inclusion of the auxiliary variable was required to obtain nominal level of coverage probability in both MAR2A and MNAR (Appendix Table A4 and Appendix Figure A3). 


\section{MULTIPLE IMPUTATION IN PROPENSITY SCORE MATCHING}
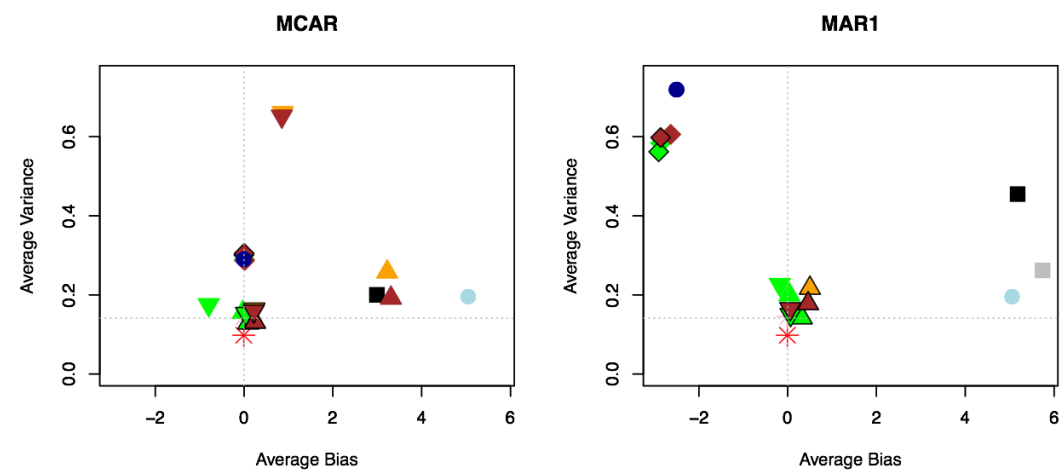

* Full Data (regression)

Full Data (PSM)

Complete Variable Analys - Mean Imputation

Missing Indicator
Ml-derPassive INT-within

Average Bias
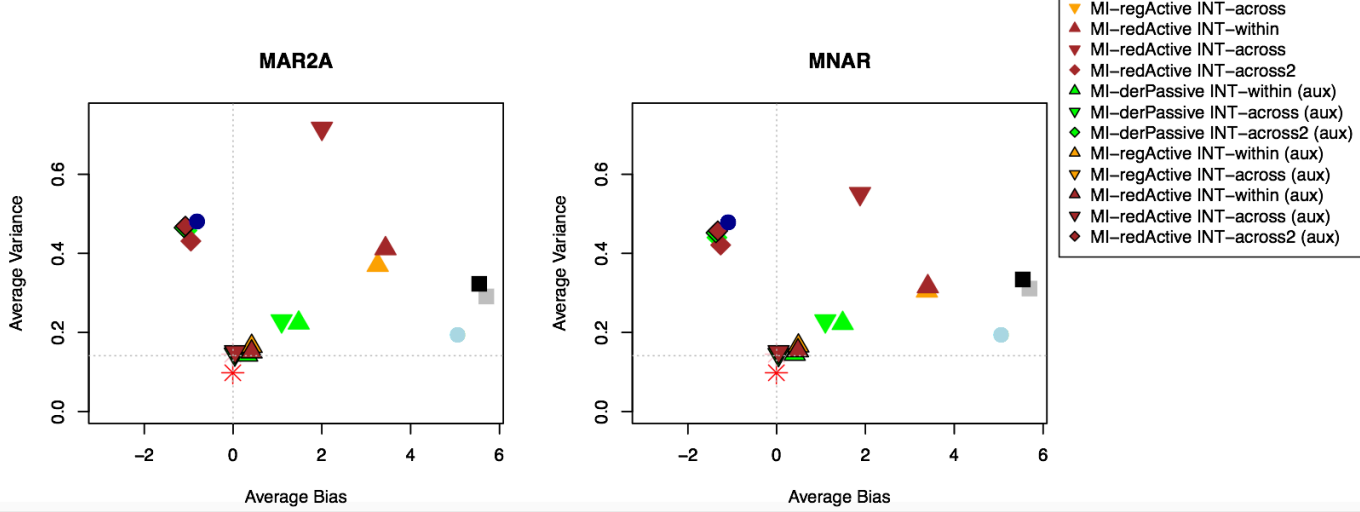

Figure 3. Average bootstrap variance vs. average bias computed over 1,000 simulated datasets, by multiple imputation estimation and integration strategies for propensity score matching where $X_{2}$ was missing under MCAR, MAR1, MAR2A, and MNAR. MCAR, missing completely at random; MAR, missing at random; MNAR, missing not at random; MAR1, simple MAR; MAR2A, complex MAR.

The performance of MI strategies is shown in Appendix Table A5 and Appendix Figure A1 under a modified MAR2 scenario (MAR2B) where missingness was a function of a different auxiliary variable, $Z_{p s}$ (dichotomized), treatment, and outcome. For reference, performance of passive approaches when $Z_{p s}$ was fully observed was first evaluated. MI-derPassive INT-within achieved the lowest $\mathrm{rMSE}$ and bias $(\mathrm{rMSE}=1.032$, bias $=-0.019)$, followed by INT-across $(\mathrm{rMSE}=1.394$, bias $=-0.205)$, and INT-across $2(\mathrm{rMSE}=10.319$, bias $=-1.016)$. The bootstrapped standard error was the largest in INT-across 2 (0.682) and comparable for INT-within (0.378) and INT-across (0.381). When $Z_{p s}$ was partially observed, MI-derPassive and MI-regPassive were largely comparable. Although INT-across methods yielded the smallest bootstrap standard error, INT-within methods resulted in smaller bias and MSE. While imputing $X_{2}$ before or after $Z_{p s}$ 
affected the resulting bias, bootstrap standard error and MSE, the order did not change our conclusions on the best performing imputation and integration MI strategies as mentioned above.

\section{Comparison across MDMs}

Summarizing results across all MDMs, MI-derPassive outperformed MI-redActive, followed by MI-regActive in terms of rMSE, bias, and efficiency (Table 1, Appendix Table A4, Figure 3, and Appendix Figure A3). MI-derPassive INTwithin demonstrated strong performance regardless of the presence of an auxiliary term. Even though MI-derPassive INT-across achieved the smallest rMSE in MAR2A and MNAR when auxiliary variable was included, MI-derPassive INTwithin had comparable rMSE. Inclusion of an auxiliary variable did not greatly improve properties of the top performers under MCAR and MAR1. In contrast, the performance of MI-strategies was much improved under MAR2A and MNAR, where missingness was related to the auxiliary variable.

\section{Sensitivity simulation results}

The results from the second set of simulations, where there was a reduced confounding effect - shown in Appendix Tables A8-A10 - are largely consistent with the main simulation results in sections above. Next, applying the optimal MI strategy from the main simulation study, MI-derPassive INT-within, we found that both bias and efficiency suffered with higher missing rate and inadequately specified number of multiply imputed datasets, $m$ (Appendix Table A11). When the missing rate decreased from $50 \%$ in the main simulation study to $25 \%$ and $10 \%$ under MAR1, the bias and bootstrap standard error also decreased (bias $=0.038$, 0.021 , and 0.005 , bootstrap standard error $=0.446,0.389$, and 0.365 respectively). When only 10 multiply imputed datasets were used at $50 \%$ missingness, both bias (0.096) and bootstrap standard error (0.454) increased compared to when $m=50$ (bias $=0.038$, bootstrap standard error $=0.446$ under MAR1) (Appendix Table A11). In the absence of missingness, when the study sample size was 1000,500 , and 250 , the bias $(-0.186,0.300,-0.315)$ and robust standard error $(0.524,0.715$, 1.073) increased compared to when the sample size was 2,000 in the main simulation study (bias $=-0.006$, standard error $=0.380$ ) (data not shown in tables). Nevertheless, MI-derPassive INT-within performed reasonably well in terms of both bias and efficiency. For example, under MAR1, the biases were 0.11, 0.22, 0.374 and bootstrap standard errors were $0.63,0.878,1.138$ with sample sizes 1000 , 500, and 250 respectively (Appendix Table A11). 


\section{MULTIPLE IMPUTATION IN PROPENSITY SCORE MATCHING}

\section{Case Study}

The various MI strategies discussed in our simulation study were illustrated in a real-world example. The goal of the exemplified study was to assess whether an electronic text message (e-message) intervention had an impact on treatment adherence, measured by proportion of class attendance, in the Diet Intervention Examining The Factors Interacting with Treatment Success (DIETFITS) trial (Oppezzo et al., 2019). DIETFITS investigated the effects of a healthy low-fat diet vs a healthy low-carbohydrate diet on weight change at 12 months in 609 overweight adults (Gardner et al., 2018). Throughout the study period, participants in both diet groups were given education classes designed to enhance participant adherence. The investigators found a decline in the education class attendance in the first four study cohorts after 6 months (Oppezzo et al., 2019). Thus, an emessage intervention was deployed to both arms of the fifth cohort with the goal of increasing adherence to their diet plan (Oppezzo et al., 2019). In the original study, PSM was performed to match the patients in Cohort 5 who received e-message intervention to historical controls in Cohort 1-4 and the effect was measured through a two-sampled t-test (Oppezzo et al., 2019).

Instead of replicating the original study, the statistical analysis was modified to better match our simulation design. A PS model was estimated in all 609 participants, among whom 97 received e-messages, and 512 did not (31 from Cohort 5 declined and 481 from Cohorts 1-4 did not have the chance to receive this e-messaging intervention). PS was estimated in the same way as the original study -- using logistic regression with confounding variables age, sex, race, weight change at 6 months, and proportion of attendance at 6 months (Oppezzo et al., 2019). 1:1 nearest neighbor matching was used without replacement with caliper 0.2 to match each participant who received e-messages to a control. The effect of e-messages on outcome variable, proportion of class attendance between the 6- and 12-month study endpoints, was estimated in a linear regression model adjusting for all confounding variables. To handle missing data, commonly used methods (CC, CVA, mean imputation, and missing indicator) were applied as well as MI strategies. A robust cluster variance was used for non-MI missing data methods and bootstrap variance was used for all MI-strategies.

Trial participants' characteristics are shown in Appendix Table A6. There were three PS variables with missing data: age $(5.15 \%$ and $24.80 \%$ in exposure and control groups respectively), race $(1.03 \%$ and $0.98 \%$ in exposure and control groups respectively) and weight change at 6 months $(8.25 \%$ and $26.37 \%$ in exposure and control groups respectively). Overall, 20\% patient-level data was 
LING ET AL.

Complete case analysis

Complete variable analysis

Mean imputation

Missing indicator

derPassive-within

derPassive-across

derPassive-across2

regActive-within

regActive-across

regActive-across2

redActive-within

redActive-across

redActive-across2

derPassive-within(aux)

derPassive-across(aux)

derPassive-across2(aux)

regActive-within(aux)

regActive-across(aux)

regActive-across2(aux)

redActive-within(aux)

redActive-across(aux)

redActive-across2(aux)

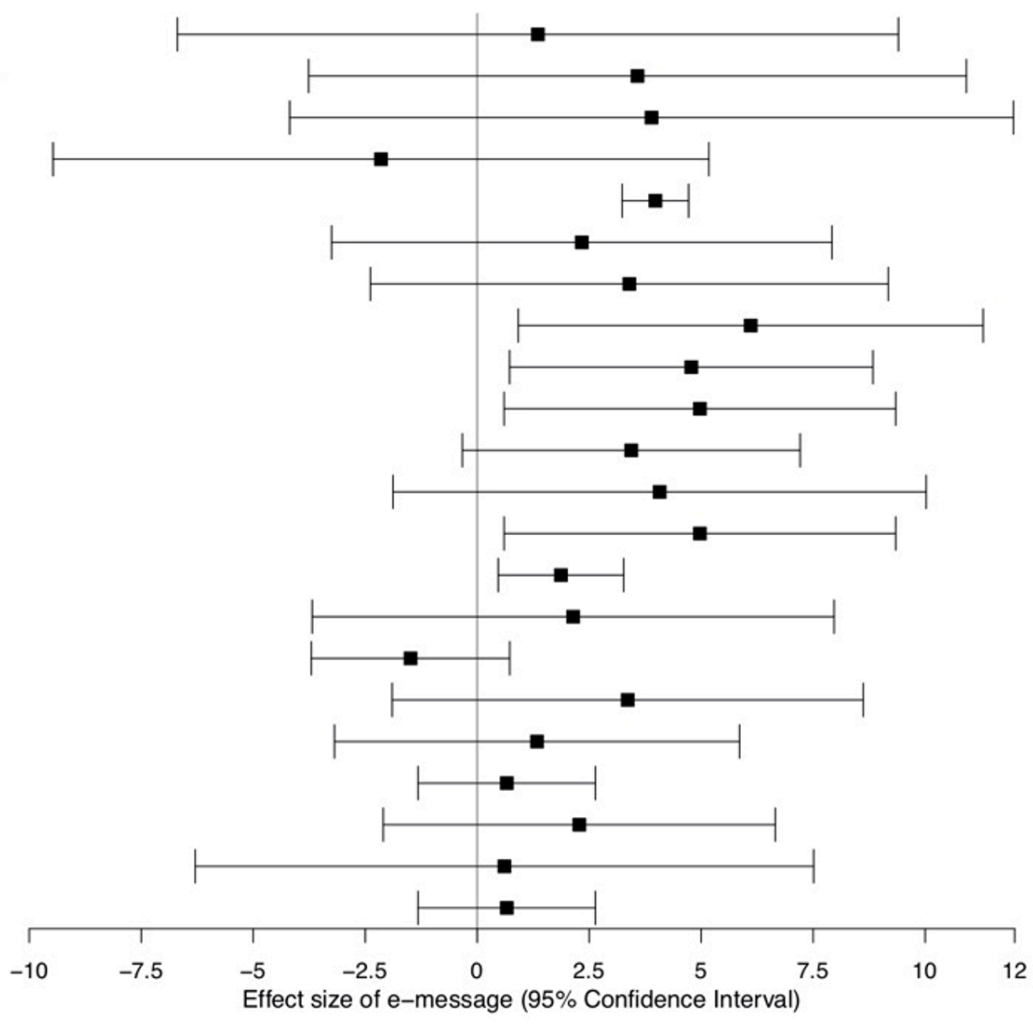

Figure 4. Application of the various missing data methods to a real-world example to assess the impact of an electronic text message (e-message) intervention on treatment adherence in the Diet Intervention Examining The Factors Interacting with Treatment Success (DIETFITS) trial, showing effect sizes of e-message with $95 \%$ confidence interval estimated using propensity score matching.

missing and $m=20$ was used in MI algorithms. Additionally, the following auxiliary variables: baseline weight, weight at 6 months, weight at 12 months, and weight change after 6 months, were included into our imputation model, as they were associated with PS variables but not of interest in our scientific model. As observed in our simulation study, MI-derPassive and MI-redActive coupled with INT-within balanced more covariates among all missing data methods (Appendix Table A7). Figure 4 shows the estimated effects of e-message and their $95 \%$ confidence intervals. The effect size of e-message intervention varied greatly from one missing data method to another, as observed in our simulations. Informed by the simulation study, findings based on MI-derPassive INT-within (both with and 


\section{MULTIPLE IMPUTATION IN PROPENSITY SCORE MATCHING}

without auxiliary variable), indicated that e-messaging had a significant impact on adherence.

\section{Discussion}

We investigated several pragmatic research questions concerning how to optimally apply MI when utilizing PSM in the presence of a partially observed confounder. We compared the performance of non-MI missing data methods that are commonly applied along with various MI-based strategies that vary both in how the PS is estimated or imputed and in how the PS is integrated into the analysis. In addition, we evaluated the impact of inclusion of an auxiliary term in the imputation model on the ranked performance of the MI strategies as well as the impact of the order of inclusion when there is more than one variable with missing data. Among the commonly applied missing data methods, CVA and single imputation methods (mean imputation and missing indicator) led to large bias in our simulation study. In contrast, $\mathrm{CC}$ was not as biased due to the use of a caliper that ensured only those subjects with closely matched PSs were included. CC did, however, suffer from loss of efficiency. There was large heterogeneity among the MI strategies considered. Based on our results, we caution applied researchers against adopting the aforementioned commonly applied missing data methods and recommend: 1) adopt MI-derPassive approaches; and 2) consider INT-within 3) use of the bootstrap to estimate variance; and 4) inclusion of key auxiliary variables in the imputation model.

Our study is important in identifying the limitations of commonly applied methods. Considerable bias and inefficiency were observed among all commonly applied methods relative to that yielded by applying PSM to the dataset without missingness. At least one of the MI-based strategies always outperformed the commonly applied methods. It is well established that balancing diagnostics are useful for PSM, but this proves difficult with commonly applied methods. For example, while $\mathrm{CC}$ can be applied using those matched pairs where balance is achieved, bias may still occur because of the observations not included due to missingness. For missing indicators, we were unable to achieve balance in the variable with missing data, as revealed in our simulations when we parsed the data by observed and missing data to evaluate balance in these respective parts. Thus, in practice, one may have a false sense of the balance as the user is only privy to assessing the balance in the observed data. Similarly, application of mean imputation distorts the distributional properties of the variable with missing data, potentially yielding a distorted view of balance when the imputed values are 
utilized in calculating the standardized differences (Appendix Table A2). Finally, for CVA, a false sense of security may be given when evaluating balance in only one variable, when exclusion of the other variable could lead to bias.

We have primarily examined the differences between passive and active MI methods when PS, a derived variable, was considered in the analysis and only partially observed. MI-derPassive methods surpassed MI-redActive approaches in almost all performance metrics across all MDMs, and MI-regActive had the worst statistical properties. Since MI-redActive can be thought of as a hybrid between MIderPassive and MI-regActive and partly mitigated the issues of MI-regActive by rederiving PS post-imputation, we hereby mainly discuss the rationale behind the poor performance of MI-regActive. To start with, MI-regActive was proposed so the entire covariance structure of all variables in the analysis, including the PS itself, could be retained. However, the covariance structure between PS and the PS variables is complex and difficult to learn using complete cases only. Unlike usual derived measures (e.g. interactions and higher order terms) that are derived as a deterministic function of other variables, PS requires estimation and its exact function will vary depending on the data considered. Thus, imputing PS together with missing covariates introduced bias into the imputation procedure and consequently the estimated treatment effect. Such bias was also reflected in the difficulty to achieve balance both in the imputed and fully observed data under $M I$ regActive (Appendix Table A3). Further, the poor estimation of the treatment effect had implications for estimates of uncertainty. The bias introduced in the estimation of the treatment effect highly varied across the bootstrap samples, leading to an increased estimate of the variation (Appendix Figure A2). Although adding an auxiliary variable reduced bias in most of the bootstrap samples, it did not help reduce bias for the extreme cases where the bias without auxiliary variable was unexpectedly high. Second, PSM is a two-stage analysis, in which after matching, PS itself is not directly used in estimating the treatment effect. In contrast, other derived variables (e.g. interactions) are usually directly involved in the regression model for treatment effect estimation. As a result, capturing the covariance structure between PS and other variables in the imputation process did not yield the same benefits as seen in the derived variables that were studied before. Third, although we only simulated two PS variables, researchers are likely to include a larger number of PS covariates with complex missing pattern. This will lead to a high overall level of missingness for PS estimation in the first step of MI-regActive, which increases the difficulty of the subsequent imputation step. Fourth, unless we specify the true relationship between PS and PS variables using an inverse logistic function (PMM was used in our simulations), which is cumbersome to implement 


\section{MULTIPLE IMPUTATION IN PROPENSITY SCORE MATCHING}

in reality, the imputation model is technically misspecified, potentially leading to bias as well.

We recommend INT-within as the optimal MI integration strategy to combine with imputation strategy MI-derPassive, for its superior performance in all evaluating metrics as well as its ability to balance covariates post-matching. While Mitra \& Reiter recommended INT-across, de Vries \& Groenwold argued that such findings were due to a combination of omitting outcome in the imputation model and a violation of positivity assumption in the PSM process (B. B. L. de Vries \& Groenwold, 2016; Mitra \& Reiter, 2016). Further, they found INT-within to yield estimators with better statistical properties (B. B. L. P. de Vries \& Groenwold, 2017). In a different setting (IPTW instead of PSM), Leyrat et. al. also demonstrated superior properties of INT-within methods over INT-across and INT-across 2 (Leyrat et al., 2019). Our results were consistent with de Vries \& Groenwold (B. B. L. P. de Vries \& Groenwold, 2017) and Leyrat and others (Leyrat et al., 2019). We also share the perspective of Leyrat and others (Leyrat et al., 2019) that it was more straightforward to assess balance in INT-within strategies and observed that the covariates were mostly balanced in both the imputed and full datasets (Appendix Table A3). Further, Leyrat and others pointed out that the INT-across and INT-across 2 produced consistent estimators only when both the observed and imputed data were balanced (Leyrat et al., 2019). We therefore paid close attention to balance diagnostics for INT-across and INT-across 2 methods but did not observe balance in both parts of the data (observed and missing) under all MDMs other than under MCAR.

An important contribution of our paper is resolution of how to estimate the variance when doing PSM and applying MI. There has been extensive but conflicting research on this topic in the context of MI and IPTW where Rubin's Rules have been recommended for INT-within by some authors (Leyrat et al., 2019; S. Seaman \& White, 2014) and a bootstrapped-based estimator was recommended by others (Qu \& Lipkovich, 2009). Relative to IPTW, however, we are faced with the additional issue of capturing the uncertainty of matching in PSM. Prior studies of MI applications in the context of PSM acknowledged this issue (Hill, 2004; Mitra \& Reiter, 2016), but only one study has explicitly stated their recommendation of a bootstrapped-based variance (B. B. L. P. de Vries \& Groenwold, 2017), although the choice was not studied comparatively or discussed fully. In our study, we found that application of Rubin's Rules when the robust cluster estimator was used for each imputed dataset overestimated the variance under INT-within approaches and underestimated it under INT-across approaches. We therefore agree with de Vries \& Groenwold (B. B. L. P. de Vries \& Groenwold, 2017) in recommending the 
bootstrapped variance, as it captures the uncertainty of PS estimation, matching procedure, and imputation process. Further, it demonstrated good performance with respect to the empirical variance. We acknowledge the lack of theoretical support for this choice, which comes with challenges, as the estimator for the treatment effect based on PSM and MI is not a smooth function. Although Abadie \& Imbens (Abadie \& Imbens, 2008) proved that the bootstrap variance is not valid in matching with replacement, their results may not be applicable in our study when matching was done without replacement, where one control unit can only be used for matching at most once (Austin \& Small, 2014). Other alternative nonparametric solutions with stronger theoretical justification, such as subsampling, has their own limitations (e.g. the need for a sufficiently large sample size and a burden on the user to appropriately select a sub-sample and replication size) (Politis \& Romano, 1994).

Auxiliary variables are often useful for adhering to a MAR MDM, but not always possible in the context of PSM. As shown in our simulation study, when missingness is related to an auxiliary variable in MAR2A, inclusion of the auxiliary variable ensured a truly MAR scenario, which would be an MNAR scenario otherwise, making it difficult to obtain statistically valid results using MI. Having the auxiliary variable in the imputation model indeed improved all statistical properties of our recommended optimal MI strategy, MI-derPassive INT-within. In reality, however, variables related to partially observed confounders may be considered confounders themselves and thus, may not exist outside of estimation of the PS. Our team has worked on studies, however, where auxiliary terms may be available. For example, in a comparative effectiveness study of anticoagulants among kidney transplant patients, a PS that balances patient characteristics may include body mass index (BMI) at treatment initiation but not BMI at transplant listing. The latter is an excellent candidate for an auxiliary variable that can aid in imputing BMI at treatment initiation as well as other PS covariates. By including a strong auxiliary variable in the imputation process, we showcased the maximal performance improvement given any auxiliary variable. In practice, the strength of auxiliary variable varies and consequently the improvement in performance may be moderate.

There are several limitations to our study. As with any simulation study, we recognize that the limited scope of our simulations may compromise generalizability. Specifically, only two binary confounders were generated, which might not reflect a real-world scenario. We adopted a simple design, following the lead of others studying similar topics, so that we could hone in on the properties of the various MI methods without extra layers of complexities (Choi et al., 2019; B. 


\section{MULTIPLE IMPUTATION IN PROPENSITY SCORE MATCHING}

B. L. P. de Vries \& Groenwold, 2017; Hill, 2004; Mitra \& Reiter, 2016; S. Seaman $\&$ White, 2014). Even though we only included a continuous outcome in our simulation, we believe our findings on the MI imputation and integration strategies are not specific to the type of outcome. In fact, we found consistencies between our findings and those from simulation studies with binary outcomes studying a similar topic, IPTW (Leyrat et al., 2019; S. Seaman \& White, 2014). Regardless, future studies using time-to-event outcomes are important, especially given the added complexity of applying MI with right-censored outcomes (Barzi \& Woodward, 2004; Desai et al., 2019; Van Buuren, Boshuizen, \& Knook, 1999; White \& Royston, 2009). We also only considered the scenario when one confounder was partially observed, whereas missingness of covariates that are not confounders, treatment or outcome was not considered. However, we recommend the same MI strategy in cases of any missingness in potential confounders (covariates that are associated with the outcome but not exposure), as potential confounders should also be included in the PS model (Austin, Grootendorst, Normand, \& Anderson, 2007; Brookhart et al., 2006). One specific caveat in our comparison among different MI integration strategies is that the two confounders were not included in the regression models in the post-matching outcome analysis for INT-across. This was infeasible under this strategy since we had multiple sets of confounders but only one set of PS. Nonetheless, the lack of inclusion likely led to some of the observed bias in the comparative performance of our MI methods. Finally, there were only main effects in the data generating mechanism and we did not explore the impact of misspecifying the correct PS models.

Overall, we have addressed an important topic - how to apply MI strategies in the presence of missing values in confounders in the context of PSM. Our work will facilitate future applied researchers' choice of optimal missing data methods in all kinds of statistical analyses that involve PSM. In addition to classical causal inference settings, our results are applicable to other types of studies that utilize PS, including those that generalize randomized clinical trial findings to real-world target populations captured in observational databases (Cole \& Stuart, 2010; Stuart, Cole, Bradshaw, \& Leaf, 2011).

\section{Glossary}

Various multiple imputation (MI) strategies for the propensity score matching (PSM) context. PS, propensity score; MICE, multivariate imputation by chained equations in $\mathrm{R}$. The recommended approach is marked by *. 


\section{LING ET AL.}

Step 1. Choosing an imputation strategy (how to obtain PS through imputation)

1. MI-passive (PS is derived after missing PS model variables are imputed)

a. $\quad *$ MI-derPassive (PS is derived after completion of MICE algorithm, within which missing PS model variables are imputed)

b. MI-regPassive (PS is derived within MICE algorithm following the step where missing PS model variables are imputed)

2. MI-active (PS is imputed together with other missing PS model variables)

a. MI-regActive (PS is imputed together with other missing PS model variables)

b. MI-redActive (PS is imputed together with other missing PS model variables and redrived post imputation)

Step 2. Choosing an integration strategy (how to integrate PS in PSM)

1. * INT-within (PSM is conducted $\mathrm{m}$ times within each multiply imputed dataset and results are summarized using Rubin's Rules)

2. INT-across (PSM is conducted after averaging PS across the PSs obtained from the $\mathrm{m}$ imputed datasets)

3. INT-across2 (PSM is conducted after calculating the PS from a model where coefficients for the PS model are averaged across the $\mathrm{m}$ imputed datasets)

Step 3. Choosing a variance estimator

1. Robust cluster variance estimator

2. * Bootstrap-based variance estimator 


\section{MULTIPLE IMPUTATION IN PROPENSITY SCORE MATCHING}

\section{References}

Abadie, A., \& Imbens, G. W. (2006). Large sample properties of matching estimators for average treatment effects. Econometrica, 74(1), 235-267. https://doi.org/10.1111/j.1468-0262.2006.00655.x

Abadie, A., \& Imbens, G. W. (2008). On the failure of the bootstrap for matching estimators. Econometrica, 76(6), 1537-1557. https://doi.org/10.3982/ecta6474

Abadie, A., \& Imbens, G. W. (2016). Matching on the estimated propensity score. Econometrica, 84(2), 781-807. https://doi.org/10.3982/ecta11293

Abadie, A., \& Spiess, J. (2016). Robust post-matching inference. Journal of the American Statistical Association. https://doi.org/10.1080/01621459.2020.1840383

Austin, P. C. (2008). A critical appraisal of propensity-score matching in the medical literature between 1996 and 2003. Statistics in Medicine, 27(12), 2037-2049. https://doi.org/10.1002/sim.3150

Austin, P. C. (2011a). An introduction to propensity score methods for reducing the effects of confounding in observational studies. Multivariate Behavioral Research, 46(3), 399-424. https://doi.org/10.1080/00273171.2011.568786

Austin, P. C. (2011b). Optimal caliper widths for propensity-score matching when estimating differences in means and differences in proportions in observational studies.

Pharmaceutical Statistics, 10(2), 150-161. https://doi.org/10.1002/pst.433

Austin, P. C., Grootendorst, P., Normand, S.-L. T., \& Anderson, G. M. (2007). Conditioning on the propensity score can result in biased estimation of common measures of treatment effect: a Monte Carlo study. Statistics in Medicine, 26(4), 754-768. https://doi.org/10.1002/sim.2618

Austin, P. C., \& Small, D. S. (2014). The use of bootstrapping when using propensity-score matching without replacement: A simulation study. Statistics in Medicine, 33(24), 4306-4319. https://doi.org/10.1002/sim.6276

Azur, M. J., Stuart, E. A., Frangakis, C., \& Leaf, P. J. (2011). Multiple imputation by chained equations: what is it and how does it work? International Journal of Methods in Psychiatric Research, 20(1), 40-49. https://doi.org/10.1002/mpr.329

Barzi, F., \& Woodward, M. (2004). Imputations of missing values in practice: results from imputations of serum cholesterol in 28 cohort studies. American Journal of Epidemiology, 160(1), 34-45. https://doi.org/10.1093/aje/kwh175

Brand, J., van Buuren, S., le Cessie, S., \& van den Hout, W. (2019). Combining multiple imputation and bootstrap in the analysis of cost-effectiveness trial data. Statistics in Medicine, 38(2), 210-220. https://doi.org/10.1002/sim.7956 


\section{LING ET AL.}

Brookhart, M. A., Schneeweiss, S., Rothman, K. J., Glynn, R. J., Avorn, J., \& Stürmer, T. (2006). Variable selection for propensity score models. American Journal of Epidemiology, 163(12), 1149-1156. https://doi.org/10.1093/aje/kwj149

Carpenter, J., \& Kenward, M. (2012). Multiple imputation and its application. John Wiley \& Sons. https://doi.org/10.1002/9781119942283

Choi, J., Dekkers, O. M., \& le Cessie, S. (2019). A comparison of different methods to handle missing data in the context of propensity score analysis. European Journal of Epidemiology, 34(1), 23-36. https://doi.org/10.1007/s10654-018-0447-z

Cochran, W. G., \& Rubin, D. B. (1973). Controlling bias in observational studies: A review. Sankhya: The Indian Journal of Statistics, Series A, 35, 417-446.

Cole, S. R., \& Stuart, E. A. (2010). Generalizing evidence from randomized clinical trials to target populations: The ACTG 320 trial. American Journal of Epidemiology, 172(1), 107-115. https://doi.org/10.1093/aje/kwq084

Collins, L. M., Schafer, J. L., \& Kam, C.-M. (2001). A comparison of inclusive and restrictive strategies in modern missing data procedures. Psychological Methods, 6(4), 330. https://doi.org/10.1037/1082-989x.6.4.330

Concato, J., Shah, N., \& Horwitz, R. I. (2000). Randomized, controlled trials, observational studies, and the hierarchy of research designs. New England Journal of Medicine, 342(25), 1887-1892. https://doi.org/10.1056/nejm200006223422507

Crump, R. K., Hotz, V. J., Imbens, G. W., \& Mitnik, O. A. (2009). Dealing with limited overlap in estimation of average treatment effects. Biometrika, 96(1), 187-199. https://doi.org/10.1093/biomet/asn055

D’Agostino Jr, R. B. (2004). Propensity score estimation with missing data. In: A. Gelman \& X.-L. Meng, Eds. Applied Bayesian Modeling and Causal Inference from Incomplete-Data Perspectives: An Essential Journey with Donald Rubin's Statistical Family (Wiley Series in Probability and Statistics). John Wiley \& Sons., pp. 163-174. https://doi.org/10.1002/0470090456.ch15

D’Agostino Jr, R. B., \& Rubin, D. B. (2000). Estimating and using propensity scores with partially missing data. Journal of the American Statistical Association, 95(451), 749-759. https://doi.org/10.1080/01621459.2000.10474263

D'Agostino, R. B. (1998). Propensity score methods for bias reduction in the comparison of a treatment to a non-randomized control group. Statistics in Medicine, 17(19), 2265-2281. https://doi.org/10.1002/(sici)1097-

0258(19981015)17:19\&1t;2265::aid-sim918\&gt;3.0.co;2-b 


\section{MULTIPLE IMPUTATION IN PROPENSITY SCORE MATCHING}

de Vries, B. B. L., \& Groenwold, R. H. H. (2016). Comments on propensity score matching following multiple imputation. Statistical Methods in Medical Research, 25(6), 3066-3068. https://doi.org/10.1177/0962280216674296

de Vries, B. B. L. P., \& Groenwold, R. H. H. (2017). A comparison of two approaches to implementing propensity score methods following multiple imputation. Epidemiology, Biostatistics and Public Health, 14(4), e12630-1-e12630-21.

Desai, M., Mitani, A. A., Bryson, S. W., \& Robinson, T. (2016). Multiple Imputation When Rate of Change Is The Outcome of Interest. Journal of Modern Applied Statistical Methods, 15(1), 160-192. https://doi.org/10.22237/jmasm/1462075740

Desai, M., Montez-Rath, M. E., Kapphahn, K., Joyce, V. R., Mathur, M. B., Garcia, A., ... Owens, D. K. (2019). Missing data strategies for time-varying confounders in comparative effectiveness studies of non-missing time-varying exposures and rightcensored outcomes. Statistics in Medicine, 38(17), 3204-3220.

https://doi.org/10.1002/sim.8174

Efron, B., \& Tibshirani, R. J. (1994). An introduction to the bootstrap. CRC Press. https://doi.org/10.1201/9780429246593

Fleurence, R. L., Naci, H., \& Jansen, J. P. (2010). The critical role of observational evidence in comparative effectiveness research. Health Affairs, 29(10), 1826-1833. https://doi.org/10.1377/hlthaff.2010.0630

Gardner, C. D., Trepanowski, J. F., Del Gobbo, L. C., Hauser, M. E., Rigdon, J., Ioannidis, J. P. A., ... King, A. C. (2018). Effect of low-fat vs low-carbohydrate diet on 12-month weight loss in overweight adults and the association with genotype pattern or insulin secretion: the DIETFITS randomized clinical trial. JAMA, 319(7), 667-679. https://doi.org/10.1001/jama.2018.0245

Goodman, S. N., Schneeweiss, S., \& Baiocchi, M. (2017). Using design thinking to differentiate useful from misleading evidence in observational research. JAMA, 317(7), 705-707. https://doi.org/10.1001/jama.2016.19970

Granger, E., Sergeant, J. C., \& Lunt, M. (2019). Avoiding pitfalls when combining multiple imputation and propensity scores. Statistics in Medicine, 38(26), 5120-5132. https://doi.org/10.1002/sim.8355

Hill, J. (2004). Reducing Bias in Treatment Effect Estimation in Observational Studies Suffering from Missing Data. Columbia University Institute for Social Social and Economic Research and Policy (ISERP) Working Paper 04-01, (January). https://doi.org/10.7916/D8B85G11 


\section{LING ET AL.}

Hill, J., \& Reiter, J. P. (2006). Interval estimation for treatment effects using propensity score matching. Statistics in Medicine, 25(13), 2230-2256.

https://doi.org/10.1002/sim.2277

Ho, D. E., Imai, K., King, G., \& Stuart, E. A. (2007). Matching as nonparametric preprocessing for reducing model dependence in parametric causal inference. Political Analysis, 15(3), 199-236. https://doi.org/10.1093/pan/mp1013

Holcomb, J. B., Del Junco, D. J., Fox, E. E., Wade, C. E., Cohen, M. J., Schreiber, M. A., ... Others. (2013). The prospective, observational, multicenter, major trauma transfusion (PROMMTT) study: comparative effectiveness of a time-varying treatment with competing risks. JAMA Surgery, 148(2), 127-136.

https://doi.org/10.1001/2013.jamasurg.387

Ibrahim, J. G., Lipsitz, S. R., \& Chen, M.-H. (1999). Missing covariates in generalized linear models when the missing data mechanism is non-ignorable. Journal of the Royal Statistical Society: Series B (Statistical Methodology), 61(1), 173-190. https://doi.org/10.1111/1467-9868.00170

Imbens, G. W. (2004). Nonparametric estimation of average treatment effects under exogeneity: A review. Review of Economics and Statistics, 86(1), 4-29. https://doi.org/10.1162/003465304323023651

Lauer, M. S., \& Collins, F. S. (2010). Using science to improve the nation's health system: NIH's commitment to comparative effectiveness research. JAMA, 303(21), 2182-2183. https://doi.org/10.1001/jama.2010.726

Lechner, M. (2002). Some practical issues in the evaluation of heterogeneous labour market programmes by matching methods. Journal of the Royal Statistical Society: Series A (Statistics in Society), 165(1), 59-82. https://doi.org/10.1111/1467985x.0asp2

Leyrat, C., Seaman, S. R., White, I. R., Douglas, I., Smeeth, L., Kim, J., ... Williamson, E. J. (2019). Propensity score analysis with partially observed covariates: How should multiple imputation be used? Statistical Methods in Medical Research, 28(1), 3-19. https://doi.org/10.1177/0962280217713032

Li, F., Morgan, K. L., \& Zaslavsky, A. M. (2018). Balancing covariates via propensity score weighting. Journal of the American Statistical Association, 113(521), 390-400. https://doi.org/10.1080/01621459.2016.1260466

Li, L., \& Greene, T. (2013). A weighting analogue to pair matching in propensity score analysis. The International Journal of Biostatistics, 9(2), 215-234. https://doi.org/10.1515/ijb-2012-0030 


\section{MULTIPLE IMPUTATION IN PROPENSITY SCORE MATCHING}

Little, R. J. A., \& Rubin, D. B. (2014). Statistical analysis with missing data (Vol. 333). John Wiley \& Sons. https://doi.org/10.1002/9781119013563

Lunceford, J. K., \& Davidian, M. (2004). Stratification and weighting via the propensity score in estimation of causal treatment effects: a comparative study. Statistics in Medicine, 23(19), 2937-2960. https://doi.org/10.1002/sim.1903

Malla, L., Perera-Salazar, R., McFadden, E., Ogero, M., Stepniewska, K., \& English, M. (2018). Handling missing data in propensity score estimation in comparative effectiveness evaluations: a systematic review. Journal of Comparative Effectiveness Research, 7(3), 271-279. https://doi.org/10.2217/cer-2017-0071

McCullagh, P. (2018). Generalized linear models. Routledge. https://doi.org/10.1201/9780203753736

Meng, X.-L. (1994). Multiple-imputation inferences with uncongenial sources of input. Statistical Science, 9(4), 538-558. https://doi.org/10.1214/ss/1177010269

Mitani, A. A., Kurian, A. W., Das, A. K., \& Desai, M. (2015). Navigating choices when applying multiple imputation in the presence of multi-level categorical interaction effects. Statistical Methodology, 27, 82-99. https://doi.org/10.1016/j.stamet.2015.06.001

Mitra, R., \& Reiter, J. P. (2016). A comparison of two methods of estimating propensity scores after multiple imputation. Statistical Methods in Medical Research, 25(1), 188-204. https://doi.org/10.1177/0962280212445945

Moons, K. G. M., Donders, R. A. R. T., Stijnen, T., \& Harrell Jr, F. E. (2006). Using the outcome for imputation of missing predictor values was preferred. Journal of Clinical Epidemiology, 59(10), 1092-1101. https://doi.org/10.1016/j.jclinepi.2006.01.009

Morris, T. P., White, I. R., \& Crowther, M. J. (2019). Using simulation studies to evaluate statistical methods. Statistics in Medicine, 38(11), 2074-2102.

https://doi.org/10.1002/sim.8086

Oppezzo, M. A., Stanton, M. V, Garcia, A., Rigdon, J., Berman, J. R., \& Gardner, C. D. (2019). To Text or Not to Text: Electronic Message Intervention to Improve Treatment Adherence Versus Matched Historical Controls. JMIR MHealth and UHealth, 7(4), e11720. https://doi.org/10.2196/11720

Politis, D. N., \& Romano, J. P. (1994). Large sample confidence regions based on subsamples under minimal assumptions. The Annals of Statistics, 22(4), 2031-2050. https://doi.org/10.1214/aos/1176325770

Qu, Y., \& Lipkovich, I. (2009). Propensity score estimation with missing values using a multiple imputation missingness pattern (MIMP) approach. Statistics in Medicine, 28(9), 1402-1414. https://doi.org/10.1002/sim.3549 


\section{LING ET AL.}

R Core Team. (2018). R: A Language and Environment for Statistical Computing. Vienna, Austria.

Rosenbaum, P. R. (1987). Model-based direct adjustment. Journal of the American Statistical Association, 82(398), 387-394. https://doi.org/10.1080/01621459.1987.10478441

Rosenbaum, P. R., \& Rubin, D. B. (1983). The central role of the propensity score in observational studies for causal effects. Biometrika, 70(1), 41-55. https://doi.org/10.1093/biomet/70.1.41

Rosenbaum, P. R., \& Rubin, D. B. (1984). Reducing bias in observational studies using subclassification on the propensity score. Journal of the American Statistical Association, 79(387), 516-524. https://doi.org/10.1080/01621459.1984.10478078

Rosenbaum, P. R., \& Rubin, D. B. (1985a). Constructing a control group using multivariate matched sampling methods that incorporate the propensity score. The American Statistician, 39(1), 33-38. https://doi.org/10.1080/00031305.1985.10479383

Rosenbaum, P. R., \& Rubin, D. B. (1985b). The bias due to incomplete matching. Biometrics, 41(1), 103-116. https://doi.org/10.2307/2530647

Royston, P. (2009). Multiple imputation of missing values: further update of ice, with an emphasis on categorical variables. The Stata Journal, 9(3), 466-477. https://doi.org/10.1177/1536867x0900900308

Rubin, D. B. (1974). Estimating causal effects of treatments in randomized and nonrandomized studies. Journal of Educational Psychology, 66(5), 688. https://doi.org/10.1037/h0037350

Rubin, D. B. (1986). Comment: Which ifs have causal answers. Journal of the American Statistical Association, 81(396), 961-962. https://doi.org/10.2307/2289065

Rubin, D. B. (2004). Multiple imputation for nonresponse in surveys (Vol. 81). John Wiley \& Sons. https://doi.org/10.1002/9780470316696

Rubin, D. B., \& Thomas, N. (1996). Matching using estimated propensity scores: relating theory to practice. Biometrics, 52(1), 249-264. https://doi.org/10.2307/2533160

Schafer, J. L., \& Kang, J. (2008). Average causal effects from nonrandomized studies: a practical guide and simulated example. Psychological Methods, 13(4), 279-313. https://doi.org/10.1037/a0014268

Schomaker, M., \& Heumann, C. (2018). Bootstrap inference when using multiple imputation. Statistics in Medicine, 37(14), 2252-2266. https://doi.org/10.1002/sim.7654

Seaman, S. R., Bartlett, J. W., \& White, I. R. (2012). Multiple imputation of missing covariates with non-linear effects and interactions: an evaluation of statistical 


\section{MULTIPLE IMPUTATION IN PROPENSITY SCORE MATCHING}

methods. BMC Medical Research Methodology, 12(1), 46. https://doi.org/10.1186/14712288-12-46

Seaman, S., \& White, I. (2014). Inverse probability weighting with missing predictors of treatment assignment or missingness. Communications in Statistics-Theory and Methods, 43(16), 3499-3515. https://doi.org/10.1080/03610926.2012.700371

Sekhon, J. S. (2008). Multivariate and propensity score matching software with automated balance optimization: the matching package for R. Journal of Statistical Software, 42(7). https://doi.org/10.18637/jss.v042.i07

Sterne, J. A. C., White, I. R., Carlin, J. B., Spratt, M., Royston, P., Kenward, M. G., ... Carpenter, J. R. (2009). Multiple imputation for missing data in epidemiological and clinical research: potential and pitfalls. BMJ, 338(jun29 1), b2393. https://doi.org/10.1136/bmj.b2393

Stuart, E. A. (2008). Developing practical recommendations for the use of propensity scores: Discussion of 'A critical appraisal of propensity score matching in the medical literature between 1996 and 2003' by Peter Austin. Statistics in Medicine. Statistics in Medicine, 27(12), 2062-2065. https://doi.org/10.1002/sim.3207

Stuart, E. A. (2010). Matching methods for causal inference: A review and a look forward. Statistical Science: A Review. Journal of the Institute of Mathematical Statistics, 25(1), 1. https://doi.org/10.1214/09-sts313

Stuart, E. A., Cole, S. R., Bradshaw, C. P., \& Leaf, P. J. (2011). The use of propensity scores to assess the generalizability of results from randomized trials. Journal of the Royal Statistical Society: Series A (Statistics in Society), 174(2), 369-386. https://doi.org/10.1111/j.1467-985x.2010.00673.x

Vable, A. M., Kiang, M. V, Glymour, M. M., Rigdon, J., Drabo, E. F., \& Basu, S. (2019). Performance of matching methods as compared with unmatched ordinary least squares regression under constant effects. American Journal of Epidemiology, 188(7), 1345-1354. https://doi.org/10.1093/aje/kwz093

van Buuren, S, \& Groothuis-Oudshoorn, K. (2010). mice: Multivariate imputation by chained equations in R. Journal of Statistical Software, 45(3), 1-68. https://doi.org/10.18637/jss.v045.i03

Van Buuren, Stef. (2018). Flexible imputation of missing data. Chapman and Hall/CRC. https://doi.org/10.1201/b11826

Van Buuren, Stef, Boshuizen, H. C., \& Knook, D. L. (1999). Multiple imputation of missing blood pressure covariates in survival analysis. Statistics in Medicine, 18(6), 681-694. https://doi.org/10.1002/(sici)1097-0258(19990330)18:6\&1t;681::aidsim71\&gt;3.0.co;2-r 


\section{LING ET AL.}

Von Hippel, P. T. (2009). 8. How to impute interactions, squares, and other transformed variables. Sociological Methodology, 39(1), 265-291. https://doi.org/10.1111/j.1467-9531.2009.01215.x

Wan, F. (2019). Matched or unmatched analyses with propensity-score--matched data? Statistics in Medicine, 38(2), 289-300. https://doi.org/10.1002/sim.7976

White, I. R., \& Carlin, J. B. (2010). Bias and efficiency of multiple imputation compared with complete-case analysis for missing covariate values. Statistics in Medicine, 29(28), 2920-2931. https://doi.org/10.1002/sim.3944

White, I. R., \& Royston, P. (2009). Imputing missing covariate values for the Cox model. Statistics in Medicine, 28(15), 1982-1998. https://doi.org/10.1002/sim.3618

White, I. R., Royston, P., \& Wood, A. M. (2011). Multiple imputation using chained equations: issues and guidance for practice. Statistics in Medicine, 30(4), 377399. https://doi.org/10.1002/sim.4067 


\section{MULTIPLE IMPUTATION IN PROPENSITY SCORE MATCHING}

\section{Appendix A}
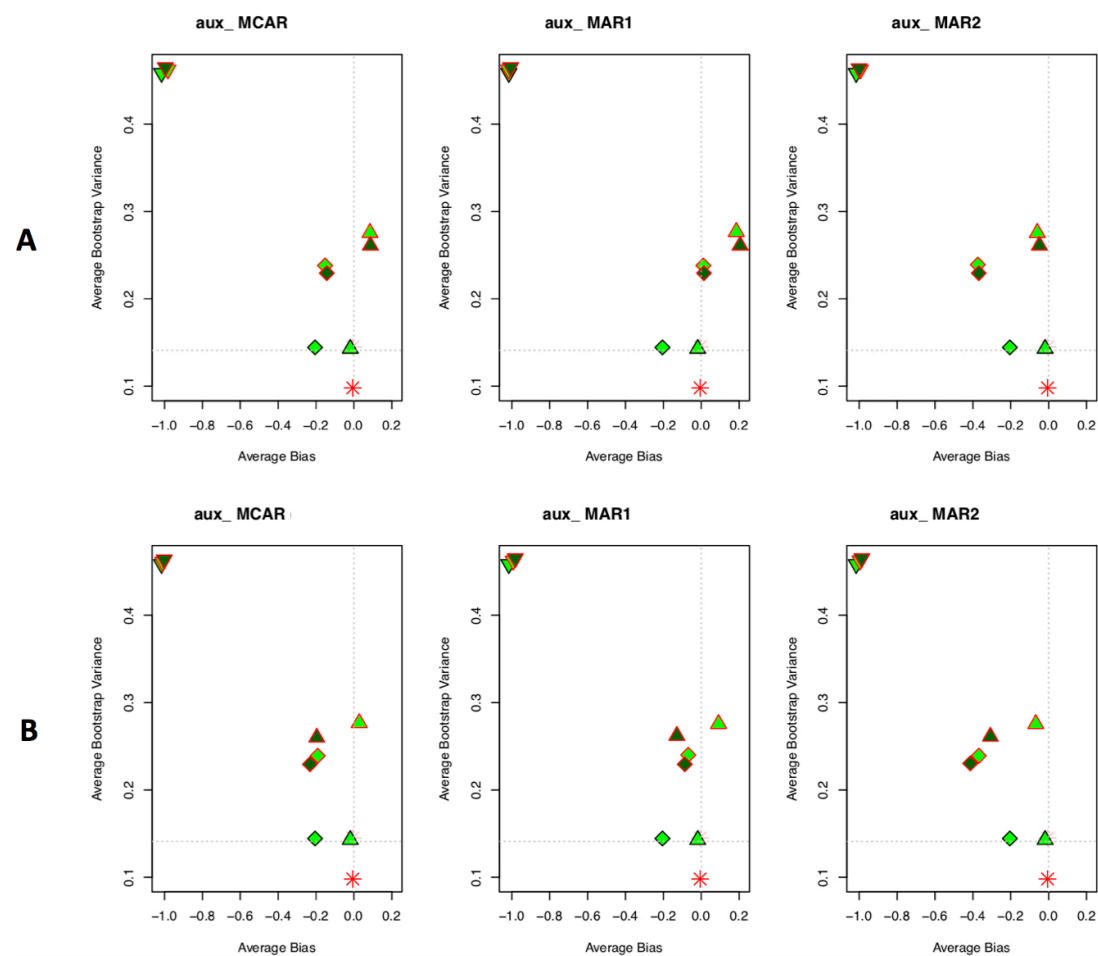

* Full Data (regression)
Full Data (PSM)

$\Delta$ MI-derPassive INT-within (aux)
$\nabla$ MI-derPassive INT-across (aux)

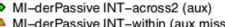
M MI-derPasssive INT--achoss (auu missining)
- MI-derPassive INT-across2 (aux missing Mi-regPassive NT- - MI-regPassive INT-across2 (aux missing)

B

Figure A1. Average bootstrap variance vs. average bias computed over 1,000 simulated datasets, by passive multiple imputation estimation and integration strategies for propensity score matching where $X_{2}$ was missing under a complex MAR (MAR2B) involving auxiliary variable $Z_{p s}$ which was missing under simple MCAR (aux_MCAR), simple MAR (aux_MAR1), or complex MAR (aux_MAR2). A. Default imputation: $X_{2}$ imputed before imputation of $Z_{p s}$; B. Reverse imputation: $Z_{p s}$ imputed before imputation of $X_{2}$. MCAR, missing completely at random; MAR, missing at random. 
LING ET AL.
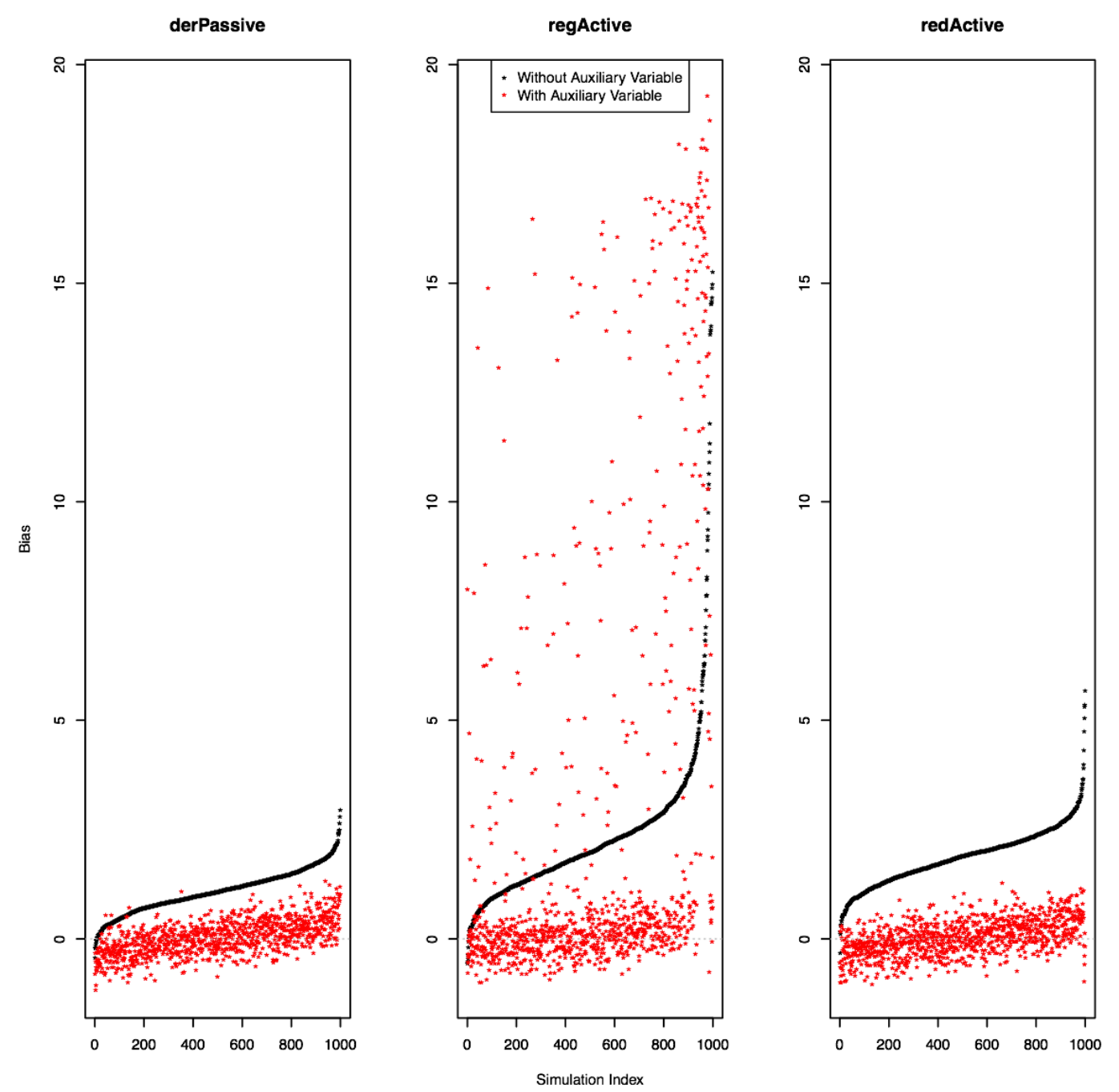

Figure A2. Illustration of bias in estimated treatment effects in 1,000 simulations MIregActive when $X_{2}$ was missing under MNAR. The bias from each simulation when auxiliary variable was not included (black dots) was ranked from the lowest (left) to the highest (right). The red dots indicate the bias from the same simulation but when the auxiliary variable was included. We can see that, including an auxiliary variable reduces bias in most cases, except in the simulations with extremely high bias (right hand side of the plot). This explains why including an auxiliary variable increased the bootstrap variance as seen Appendix Table A4. 


\section{MULTIPLE IMPUTATION IN PROPENSITY SCORE MATCHING}
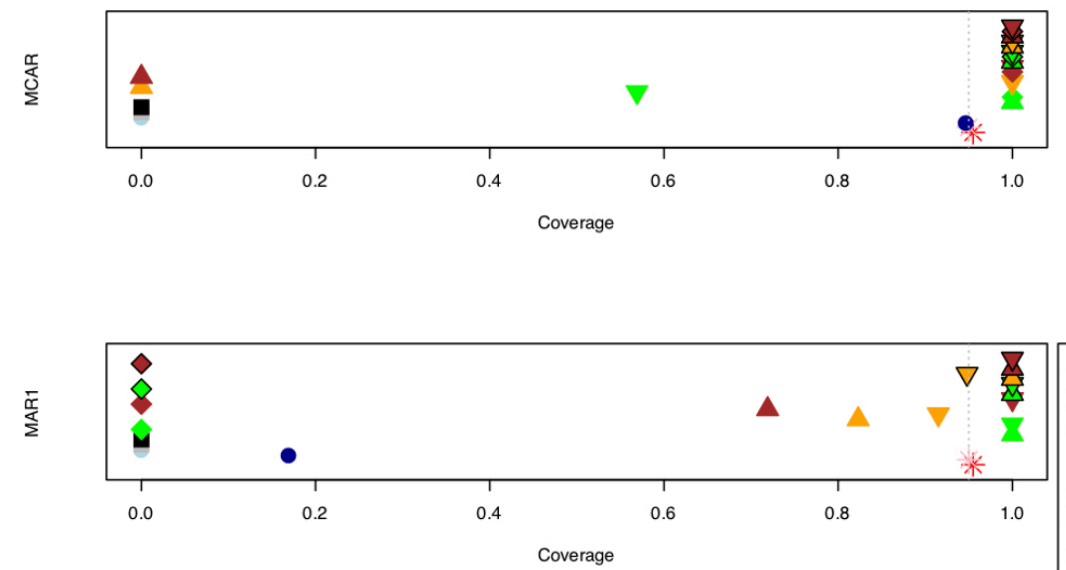

* Full Data (regression) Full Data (PSM) - Complete Case Analysis Complete Variable Analysis Mean Imputation - Missing Indicator MI-derPassive INT-within $\checkmark$ MI-derPassive INT-across - Ml-derPassive-across2 Ml-regActive INT-within MI-regActive INT-across - Ml-redActive INT-within

Ml-redActive INT-across - Ml-redActive INT-across2 $\Delta$ MI-derPassive INT-within (aux)
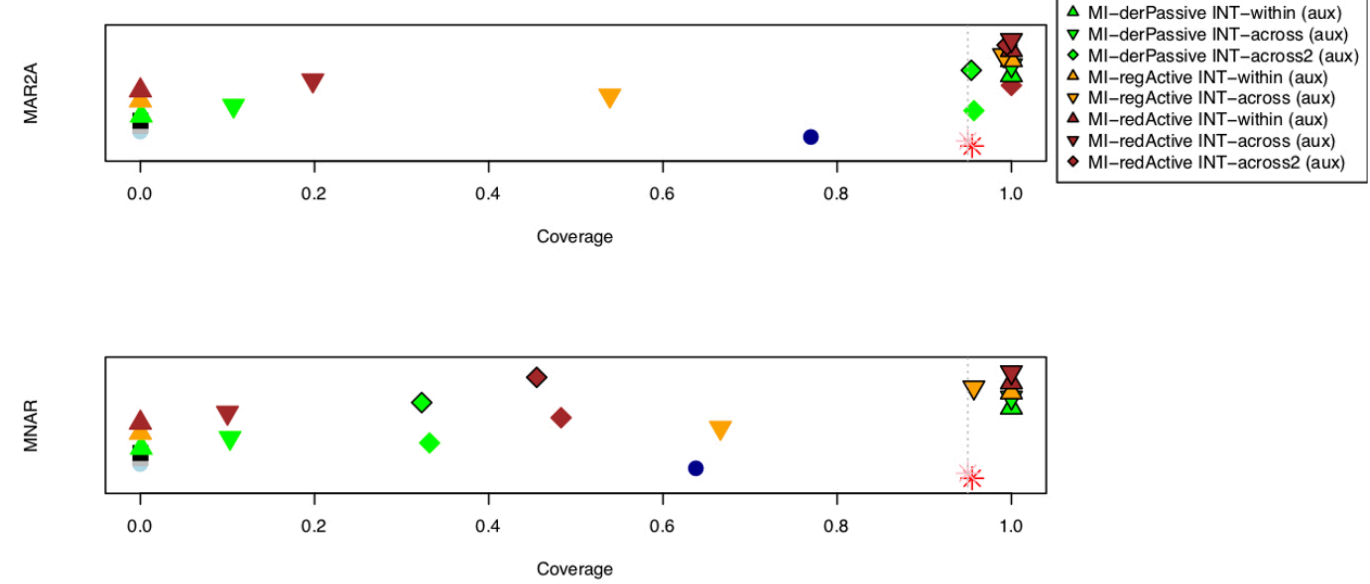

Figure A3. Coverage of various missing data methods by missing data mechanisms. 


\section{LING ET AL.}

Table A1. Comparison of the different predictors included in the imputation model for the multiple imputation approaches included.

\begin{tabular}{|c|c|c|c|c|}
\hline \multicolumn{5}{|c|}{ Scientific model: $Y \sim T+X_{1}+X_{2}$} \\
\hline \multirow{2}{*}{$\begin{array}{l}\text { Missing } \\
\text { Variable }\end{array}$} & \multicolumn{4}{|c|}{ Predictors in imputation model } \\
\hline & MI-regActive & MI-redActive $^{*}$ & MI-derPassive ${ }^{\star \star *}$ & MI-regPassive ${ }^{* * *}$ \\
\hline$X_{2}$ & $X_{1}, T, Y, P S\left(Z_{2}\right)$ & $X_{1}, T, Y, P S\left(Z_{2}\right)$ & $X_{1}, T, Y,\left(Z_{2}\right.$ or $\left.Z_{p s}\right)$ & $X_{1}, T, Y, Z_{p s}$ \\
\hline$P S$ & $X_{1}, X_{2}, T, Y\left(Z_{2}\right)$ & $X_{1}, X_{2}, T, Y\left(Z_{2}\right)$ & - & - \\
\hline$Z^{\star \star}$ & - & - & $X_{1}, X_{2}, T, Y$ & $X_{1}, X_{2}, T, Y, P S$ \\
\hline
\end{tabular}

${ }^{*} P S$ is re-derived from $X_{1}$ and $X_{2}$ after MI procedure

${ }^{* *} Z_{2}$ was used in MI-derPassive, MI-regActive, and MI-redActive when it was fully observed. $Z_{p s}$ was used in MI-derPassive and MI-regPassive when $20 \%$ of values were missing. Under MAR2B, the order of imputation (whether $Z_{p s}$ was imputed before or after $X_{2}$ ) was also evaluated.

*** We compare and contrast MI-derPassive and MI-regPassive when $X_{2}$ and $Z_{p s}$ are both missing below (the case where $X_{2}$ is imputed before $\left.Z_{p s}\right)$ :

In MI-derPassive:

1) Within Multivariate Imputation via Chained Equations (MICE) algorithm:

a. Impute $X_{2}$ using $X_{1}, T, Y$, and $Z_{p s}$

b. Impute $Z_{p s}$ using $X_{1}, X_{2}, T$, and $Y$

c. Repeat until algorithm converges

2) Estimate $P S$ using $X_{1}$ and $X_{2}$

In MI-regPassive:

1) Within MICE algorithm

a. Impute $X_{2}$ using $X_{1}, T, Y$, and $Z_{p s}$

b. Estimate $P S$ from $X_{1}$ and $X_{2}$

c. Impute $Z_{p s}$ using $X_{1}, X_{2}, T, Y$, and $P S$

d. Repeat until algorithm converges 


\section{MULTIPLE IMPUTATION IN PROPENSITY SCORE MATCHING}

Table A2. Main simulation results: balance diagnosis and treatment effect estimation results using commonly applied missing data method before propensity score matching (PSM), in reference to applying PSM to full data without missingness (Monte Carlo standard errors in parentheses). $\mathrm{MDM}=$ missing data mechanism, $\mathrm{CC}=$ complete-case analysis, $\mathrm{CVA}=$ complete-variable analysis.

\begin{tabular}{|c|c|c|c|c|c|c|c|c|c|c|c|}
\hline \multirow[b]{2}{*}{ MDM } & \multirow[b]{2}{*}{ Method } & \multirow[b]{2}{*}{ Bias } & \multicolumn{2}{|c|}{ Standard Error } & \multirow[b]{2}{*}{ MSE } & \multirow[b]{2}{*}{ rMSE } & \multirow[b]{2}{*}{ Coverage } & \multirow[b]{2}{*}{$\begin{array}{r}\text { Percentage } \\
\text { Matched }\end{array}$} & \multicolumn{3}{|c|}{$\begin{array}{c}\text { Post-Matching Standardized } \\
\text { Difference }\end{array}$} \\
\hline & & & Empirical & Robust & & & & & $\begin{array}{l}\text { Underlying } \\
\text { data }\end{array}$ & $\mathrm{X} 1$ & $\mathrm{X} 2$ \\
\hline \multirow[t]{2}{*}{$\begin{array}{l}\text { Full } \\
\text { Data }\end{array}$} & NA & $\begin{array}{r}-0.006 \\
(0.012)\end{array}$ & $\begin{array}{r}0.376 \\
(0.008)\end{array}$ & $\begin{array}{r}0.380 \\
(0.001)\end{array}$ & $\begin{array}{r}0.141 \\
(0.006)\end{array}$ & 1.000 & $\begin{array}{r}0.950 \\
(0.006)\end{array}$ & 0.653 & - & 0.000 & 0.000 \\
\hline & & 0.002 & 0.513 & 0.537 & 0.262 & & 0.957 & & & & \\
\hline \multirow[t]{9}{*}{ MCAR } & $\mathrm{CC}$ & $(0.011)$ & $(0.011)$ & $(0.001)$ & $(0.007)$ & 1.857 & $(0.006)$ & $0.653^{*}$ & - & -0.001 & -0.004 \\
\hline & & 5.058 & 0.436 & 0.442 & 25.772 & & & & & & \\
\hline & CVA & $(0.013)$ & $(0.01)$ & (0) & $(0.132)$ & 182.565 & $0(0)$ & 0.960 & - & 0 & 0.947 \\
\hline & Mean & 2.985 & 0.461 & 0.447 & 9.122 & & & & & & \\
\hline & Imputation & $(0.014)$ & $(0.01)$ & $(0.001)$ & $(0.084)$ & 64.617 & $0(0)$ & 0.803 & original data & 0 & 0.546 \\
\hline & & & & & & & & & imputed data & 0 & 0 \\
\hline & $\begin{array}{l}\text { Missing } \\
\text { Indicator }\end{array}$ & $\begin{array}{r}2.973 \\
(0.016)\end{array}$ & $\begin{array}{l}0.440 \\
(0.01)\end{array}$ & $\begin{array}{r}0.446 \\
(0.001)\end{array}$ & $\begin{array}{r}9.032 \\
(0.098)\end{array}$ & 63.981 & $0(0)$ & 0.535 & full data & 0 & 0.546 \\
\hline & & & & & & & & & observed part & 0 & 0 \\
\hline & & & & & & & & & missing part & 0 & 0.946 \\
\hline \multirow[t]{8}{*}{ MAR1 } & CC & $\begin{array}{r}-2.489 \\
(0.017) \\
5.059\end{array}$ & $\begin{array}{r}0.821 \\
(0.018) \\
0.434\end{array}$ & $\begin{array}{r}0.838 \\
(0.002) \\
0.443\end{array}$ & $\begin{array}{r}6.869 \\
(0.088) \\
25.782\end{array}$ & 48.658 & $\begin{array}{r}0.157 \\
(0.012)\end{array}$ & $1.000^{*}$ & - & 0.002 & -0.001 \\
\hline & CVA & $(0.013)$ & $(0.01)$ & & $(0.131)$ & 182.634 & $0(0)$ & 0.960 & - & 0 & 0.948 \\
\hline & Mean & 5.759 & 0.573 & 0.497 & 33.498 & & & & & & \\
\hline & Imputation & $(0.017)$ & $(0.013)$ & $(0.004)$ & $(0.195)$ & 237.298 & $0(0)$ & 0.735 & original data & 0 & 1.206 \\
\hline & & & & & & & & & imputed data & 0 & 0.095 \\
\hline & $\begin{array}{l}\text { Missing } \\
\text { Indicator }\end{array}$ & $\begin{array}{r}5.201 \\
(0.025)\end{array}$ & $\begin{array}{r}0.686 \\
(0.015)\end{array}$ & $\begin{array}{r}0.674 \\
(0.001)\end{array}$ & $\begin{array}{l}27.517 \\
(0.267)\end{array}$ & 194.929 & $0(0)$ & 0.271 & full data & 0 & 0.936 \\
\hline & & & & & & & & & observed part & 0 & 0.034 \\
\hline & & & & & & & & & missing part & 0 & 1.517 \\
\hline \multirow[t]{8}{*}{$\begin{array}{l}\text { MAR2 } \\
\text { A }\end{array}$} & CC & $\begin{array}{r}-0.815 \\
(0.015)\end{array}$ & $\begin{array}{r}0.726 \\
(0.016)\end{array}$ & $\begin{array}{r}0.682 \\
(0.002)\end{array}$ & $\begin{array}{l}1.191 \\
(0.03)\end{array}$ & 8.435 & $\begin{array}{r}0.764 \\
(0.013)\end{array}$ & $1^{*}$ & - & -0.001 & 0.001 \\
\hline & & 5.059 & 0.443 & 0.442 & 25.788 & & & & & & \\
\hline & CVA & $(0.013)$ & $(0.01)$ & $(0)$ & $(0.134)$ & 182.680 & $0(0)$ & 0.960 & - & 0 & 0.947 \\
\hline & Mean & 5.706 & 0.574 & 0.535 & 32.886 & & & & & & \\
\hline & Imputation & $(0.017)$ & $(0.013)$ & $(0.002)$ & $(0.196)$ & 232.962 & $0(0)$ & 0.602 & original data & 0 & 1.080 \\
\hline & & & & & & & & & imputed data & 0 & 0.044 \\
\hline & $\begin{array}{l}\text { Missing } \\
\text { Indicator }\end{array}$ & $\begin{array}{r}5.528 \\
(0.021)\end{array}$ & $\begin{array}{r}0.578 \\
(0.013)\end{array}$ & $\begin{array}{r}0.568 \\
(0.001)\end{array}$ & $\begin{array}{l}30.890 \\
(0.237)\end{array}$ & 218.819 & $0(0)$ & 0.352 & full data & -0.001 & 0.954 \\
\hline & & & & & & & & & observed part & -0.001 & 0 \\
\hline
\end{tabular}




\section{LING ET AL.}

\begin{tabular}{|c|c|c|c|c|c|c|c|c|c|c|c|}
\hline & & & & & & & & & missing part & -0.001 & 1.704 \\
\hline \multirow{10}{*}{ MNAR } & & -1.084 & 0.675 & 0.682 & 1.629 & & 0.631 & & & & \\
\hline & CC & $(0.014)$ & $(0.015)$ & $(0.002)$ & $(0.033)$ & 11.538 & $(0.015)$ & $1^{*}$ & - & -0.005 & -0.004 \\
\hline & & 5.059 & 0.435 & 0.443 & 25.783 & & & & & & \\
\hline & CVA & $(0.013)$ & $(0.01)$ & & $(0.132)$ & 182.646 & $0(0)$ & 0.960 & - & 0 & 0.947 \\
\hline & Mean & 5.689 & 0.592 & 0.559 & 32.711 & & & & & & \\
\hline & Imputation & $(0.018)$ & $(0.013)$ & $(0.001)$ & $(0.202)$ & 231.723 & $0(0)$ & 0.557 & original data & 0 & 1.078 \\
\hline & & & & & & & & & imputed data & 0 & 0.045 \\
\hline & $\begin{array}{l}\text { Missing } \\
\text { Indicator }\end{array}$ & $\begin{array}{r}5.534 \\
(0.021)\end{array}$ & $\begin{array}{r}0.582 \\
(0.013)\end{array}$ & $\begin{array}{r}0.577 \\
(0.001)\end{array}$ & $\begin{array}{l}30.958 \\
(0.239)\end{array}$ & 219.305 & $0(0)$ & 0.345 & full data & 0 & 0.962 \\
\hline & & & & & & & & & observed part & 0 & 0 \\
\hline & & & & & & & & & missing part & 0 & 1.725 \\
\hline
\end{tabular}

${ }^{*}$ The percentage of treated patients being matched using CC is calculated from complete cases. 


\section{MULTIPLE IMPUTATION IN PROPENSITY SCORE MATCHING}

Table A3. Main simulation results: percentage of treated subjects matched and standardized difference of covariates after matching in various multiple imputation (MI) strategies. For INT-across and INT-across2, standardized differences were also calculated on the observed and missing part of the imputed data separately. MDM=missing data mechanism.

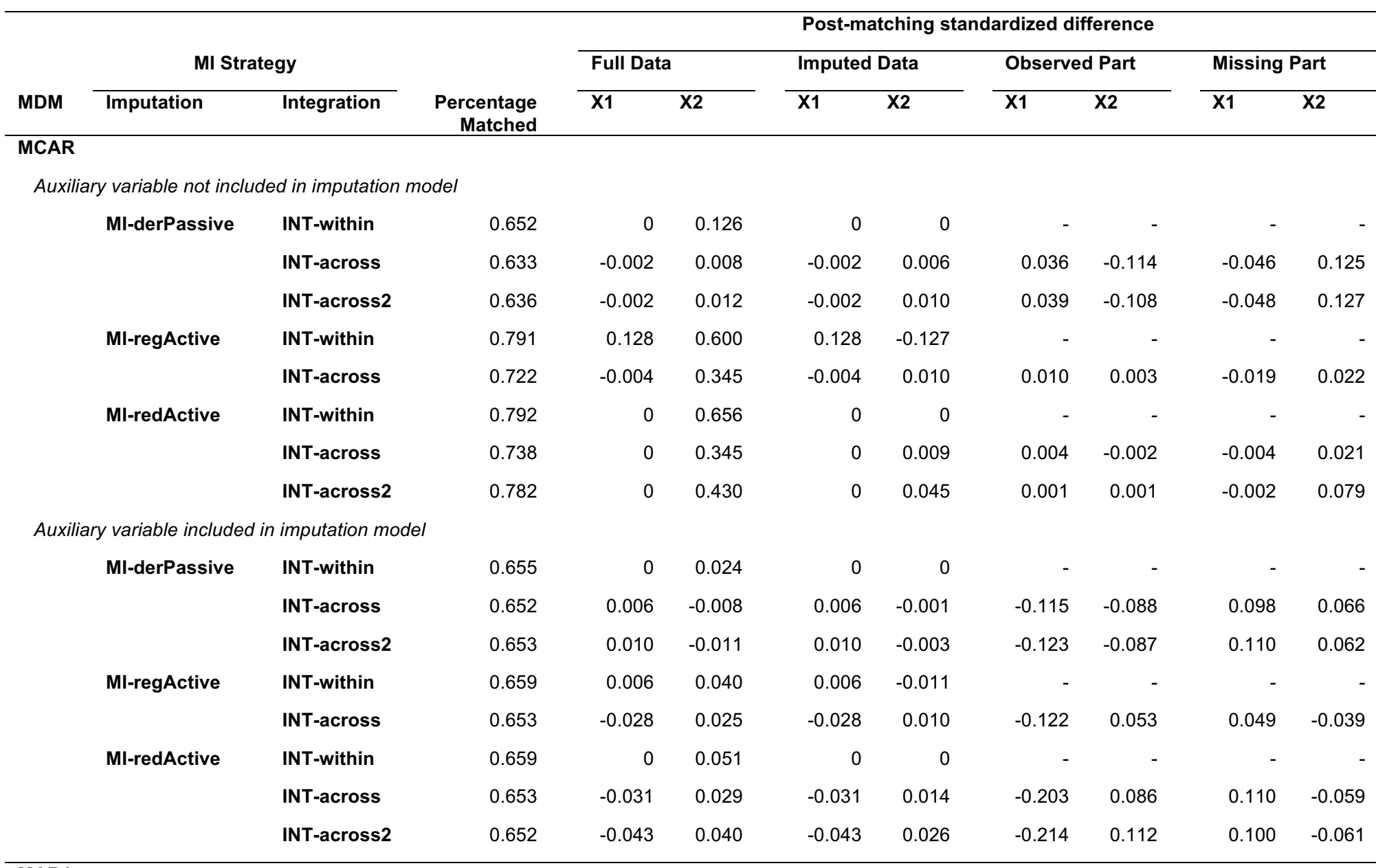

Auxiliary variable not included in imputation model

\begin{tabular}{|c|c|c|c|c|c|c|c|c|c|c|}
\hline MI-derPassive & INT-within & 0.656 & 0 & 0.114 & 0 & 0 & - & - & - & - \\
\hline & INT-across & 0.617 & 0.002 & -0.028 & 0.002 & -0.015 & -0.748 & -1.105 & 0.205 & 0.647 \\
\hline
\end{tabular}




\section{LING ET AL.}

\begin{tabular}{|c|c|c|c|c|c|c|c|c|c|c|}
\hline & INT-across2 & 0.621 & 0.008 & -0.034 & 0.008 & -0.021 & -0.746 & -1.109 & 0.201 & 0.645 \\
\hline \multirow[t]{2}{*}{ MI-regActive } & INT-within & 0.877 & 0.368 & 0.752 & 0.368 & 0.322 & - & - & - & - \\
\hline & INT-across & 0.666 & 0.562 & 0.531 & 0.562 & 0.344 & -0.160 & -0.397 & 0.714 & 0.708 \\
\hline \multirow[t]{3}{*}{ MI-redActive } & INT-within & 0.772 & 0 & 0.483 & 0 & -0.006 & - & - & - & - \\
\hline & INT-across & 0.628 & 0.002 & 0.031 & 0.002 & -0.178 & -0.746 & -1.013 & 0.241 & 0.466 \\
\hline & INT-across2 & 0.670 & 0.003 & 0.219 & 0.003 & -0.029 & -0.780 & -0.84 & 0.260 & 0.517 \\
\hline \multicolumn{11}{|c|}{ Auxiliary variable included in imputation model } \\
\hline \multirow[t]{3}{*}{ MI-derPassive } & INT-within & 0.666 & 0 & 0.065 & 0 & -0.001 & - & - & - & - \\
\hline & INT-across & 0.647 & 0.026 & -0.031 & 0.026 & -0.069 & -0.895 & -1.010 & 0.467 & 0.404 \\
\hline & INT-across2 & 0.652 & 0.022 & -0.027 & 0.022 & -0.060 & -0.953 & -0.974 & 0.519 & 0.375 \\
\hline \multirow[t]{2}{*}{ MI-regActive } & INT-within & 0.785 & 0.351 & 0.420 & 0.351 & 0.338 & - & - & - & - \\
\hline & INT-across & 0.745 & 0.386 & 0.382 & 0.386 & 0.321 & -0.672 & -0.663 & 0.856 & 0.741 \\
\hline \multirow[t]{3}{*}{ MI-redActive } & INT-within & 0.669 & 0 & 0.085 & 0 & -0.001 & - & - & - & - \\
\hline & INT-across & 0.643 & 0.012 & -0.015 & 0.012 & -0.069 & -0.921 & -1.008 & 0.460 & 0.405 \\
\hline & INT-across2 & 0.651 & 0.013 & -0.016 & 0.013 & -0.061 & -0.972 & -0.982 & 0.506 & 0.384 \\
\hline
\end{tabular}

MAR2A

Auxiliary variable not included in imputation model

\begin{tabular}{|c|c|c|c|c|c|c|c|c|c|c|}
\hline \multirow[t]{3}{*}{ MI-derPassive } & INT-within & 0.754 & 0 & 0.379 & 0 & 0 & - & - & - & \\
\hline & INT-across & 0.686 & 0 & 0.146 & 0 & -0.017 & -0.390 & -1.182 & 0.226 & 0.558 \\
\hline & INT-across2 & 0.695 & 0 & 0.171 & 0 & -0.016 & -0.392 & -1.185 & 0.212 & 0.563 \\
\hline \multirow[t]{2}{*}{ MI-regActive } & INT-within & 0.881 & 0.076 & 0.698 & 0.076 & 0.078 & - & - & - & \\
\hline & INT-across & 0.571 & 0.026 & 0.428 & 0.026 & 0.021 & -0.038 & -0.472 & 0.020 & 0.204 \\
\hline \multirow[t]{3}{*}{ MI-redActive } & INT-within & 0.863 & 0 & 0.656 & 0 & 0 & - & - & - & \\
\hline & INT-across & 0.734 & 0 & 0.348 & 0 & -0.095 & -0.331 & -0.990 & 0.107 & 0.221 \\
\hline & INT-across2 & 0.780 & 0 & 0.396 & 0 & -0.090 & -0.403 & -1.061 & 0.140 & 0.245 \\
\hline \multicolumn{11}{|c|}{ ry variable included in imputation model } \\
\hline \multirow[t]{3}{*}{ MI-derPassive } & INT-within & 0.669 & 0 & 0.071 & 0 & -0.001 & - & - & - & \\
\hline & INT-across & 0.652 & -0.014 & 0.013 & -0.014 & -0.030 & -0.441 & -1.283 & 0.294 & 0.546 \\
\hline & INT-across2 & 0.653 & -0.025 & 0.023 & -0.025 & -0.019 & -0.473 & -1.257 & 0.308 & 0.541 \\
\hline
\end{tabular}




\section{MULTIPLE IMPUTATION IN PROPENSITY SCORE MATCHING}

\begin{tabular}{lllrrrrrrrrr} 
MI-regActive & INT-within & 0.729 & 0.122 & 0.248 & 0.122 & 0.166 & - & - \\
& INT-across & 0.701 & 0.131 & 0.177 & 0.131 & 0.126 & -0.374 & -1.061 & 0.510 & 0.614 \\
& MI-redActive & INT-within & 0.672 & 0 & 0.084 & 0 & -0.001 & - & - \\
& INT-across & 0.652 & -0.018 & 0.017 & -0.018 & -0.032 & -0.476 & -1.230 & 0.341 & 0.480 \\
& INT-across2 & 0.652 & -0.026 & 0.024 & -0.026 & -0.023 & -0.506 & -1.203 & 0.361 & 0.465 \\
\hline
\end{tabular}

\section{MNAR}

Auxiliary variable not included in imputation model

\begin{tabular}{|c|c|c|c|c|c|c|c|c|c|c|}
\hline \multirow[t]{3}{*}{ MI-derPassive } & INT-within & 0.754 & 0 & 0.377 & 0 & 0 & - & - & - & \\
\hline & INT-across & 0.686 & 0 & 0.143 & 0 & -0.017 & -0.413 & -1.245 & 0.233 & 0.571 \\
\hline & INT-across2 & 0.694 & 0 & 0.168 & 0 & -0.017 & -0.416 & -1.247 & 0.219 & 0.576 \\
\hline \multirow[t]{2}{*}{ MI-regActive } & INT-within & 0.888 & 0.117 & 0.733 & 0.117 & 0.107 & - & - & - & - \\
\hline & INT-across & 0.524 & 0.041 & 0.437 & 0.041 & 0.032 & 0.066 & -0.410 & -0.045 & 0.179 \\
\hline \multirow[t]{3}{*}{ MI-redActive } & INT-within & 0.863 & 0 & 0.652 & 0 & 0 & - & - & - & - \\
\hline & INT-across & 0.729 & 0 & 0.329 & 0 & -0.107 & -0.346 & -1.080 & 0.093 & 0.229 \\
\hline & INT-across2 & 0.776 & 0 & 0.381 & 0 & -0.099 & -0.418 & -1.142 & 0.132 & 0.256 \\
\hline \multicolumn{11}{|c|}{ ry variable included in imputation model } \\
\hline \multirow[t]{3}{*}{ MI-derPassive } & INT-within & 0.671 & 0 & 0.079 & 0 & -0.001 & - & - & - & \\
\hline & INT-across & 0.652 & -0.012 & 0.011 & -0.012 & -0.039 & -0.457 & -1.339 & 0.301 & 0.542 \\
\hline & INT-across2 & 0.652 & -0.022 & 0.021 & -0.022 & -0.029 & -0.488 & -1.310 & 0.317 & 0.531 \\
\hline \multirow[t]{2}{*}{ MI-regActive } & INT-within & 0.747 & 0.204 & 0.300 & 0.204 & 0.208 & - & - & - & \\
\hline & INT-across & 0.709 & 0.240 & 0.246 & 0.240 & 0.184 & -0.294 & -1.027 & 0.623 & 0.636 \\
\hline \multirow[t]{3}{*}{ MI-redActive } & INT-within & 0.675 & 0 & 0.096 & 0 & -0.001 & - & - & - & \\
\hline & INT-across & 0.652 & -0.012 & 0.011 & -0.012 & -0.047 & -0.494 & -1.281 & 0.364 & 0.455 \\
\hline & INT-across2 & 0.652 & -0.020 & 0.019 & -0.020 & -0.037 & -0.528 & -1.251 & 0.387 & 0.441 \\
\hline
\end{tabular}




\section{LING ET AL.}

Table A4. Main simulation results: bias, standard error mean squared error (MSE), relative mean squared error (rMSE), and coverage (calculated using bootstrap standard error) results using various multiple imputation (MI) strategies in MCAR, MAR1, and MNAR. (Monte Carlo standard errors in parentheses).

\begin{tabular}{|c|c|c|c|c|c|c|c|}
\hline \multicolumn{2}{|c|}{ MI Strategy } & \multirow[b]{2}{*}{ Bias } & \multicolumn{2}{|c|}{ Standard Error } & \multirow[b]{2}{*}{ MSE } & \multirow[b]{2}{*}{ rMSE } & \multirow[b]{2}{*}{ Coverage } \\
\hline Imputation & Integration & & Empirical & Bootstrap & & & \\
\hline \multicolumn{8}{|l|}{ MCAR } \\
\hline \multicolumn{8}{|c|}{ Auxiliary variable not included in imputation model } \\
\hline \multirow[t]{3}{*}{ MI-derPassive } & INT-within & $-0.024(0.012)$ & $0.381(0.009)$ & $0.395(0.001)$ & $0.146(0.006)$ & 1.034 & $1(0)$ \\
\hline & INT-across & $-0.796(0.013)$ & $0.424(0.009)$ & $0.418(0.001)$ & $0.814(0.022)$ & 5.766 & $0.569(0.016)$ \\
\hline & INT-across2 & $0.008(0.017)$ & $0.534(0.012)$ & $0.539(0.002)$ & $0.285(0.013)$ & 2.019 & $1(0)$ \\
\hline \multirow[t]{2}{*}{ MI-regActive } & INT-within & $3.224(0.015)$ & $0.489(0.011)$ & $0.508(0.002)$ & $10.636(0.103)$ & 75.345 & $0(0)$ \\
\hline & INT-across & $0.870(0.028)$ & $0.882(0.020)$ & $0.813(0.004)$ & $1.534(0.055)$ & 10.867 & $1(0)$ \\
\hline \multirow[t]{3}{*}{ MI-redActive } & INT-within & $3.309(0.013)$ & $0.405(0.009)$ & $0.438(0.001)$ & $11.113(0.087)$ & 78.724 & $0(0)$ \\
\hline & INT-across & $0.851(0.029)$ & $0.926(0.021)$ & $0.807(0.004)$ & $1.580(0.057)$ & 11.193 & $1(0)$ \\
\hline & INT-across2 & $0.012(0.017)$ & $0.541(0.012)$ & $0.536(0.001)$ & $0.293(0.013)$ & 2.076 & $1(0)$ \\
\hline \multicolumn{8}{|c|}{ Auxiliary variable included in imputation model } \\
\hline \multirow[t]{3}{*}{ MI-derPassive } & INT-within & $0.095(0.011)$ & $0.359(0.008)$ & $0.359(0.001)$ & $0.138(0.006)$ & 0.978 & $1(0)$ \\
\hline & INT-across & $0.046(0.012)$ & $0.388(0.009)$ & $0.390(0.001)$ & $0.152(0.007)$ & 1.077 & $1(0)$ \\
\hline & INT-across2 & $-0.005(0.017)$ & $0.55(0.012)$ & $0.547(0.001)$ & $0.303(0.013)$ & 2.146 & $1(0)$ \\
\hline \multirow[t]{2}{*}{ MI-regActive } & INT-within & $0.241(0.011)$ & $0.358(0.008)$ & $0.363(0.001)$ & $0.186(0.008)$ & 1.318 & $1(0)$ \\
\hline & INT-across & $0.231(0.012)$ & $0.384(0.009)$ & $0.405(0.001)$ & $0.200(0.009)$ & 1.417 & $1(0)$ \\
\hline \multirow[t]{3}{*}{ MI-redActive } & INT-within & $0.247(0.011)$ & $0.356(0.008)$ & $0.361(0.001)$ & $0.188(0.008)$ & 1.332 & $1(0)$ \\
\hline & INT-across & $0.221(0.012)$ & $0.387(0.009)$ & $0.398(0.001)$ & $0.198(0.008)$ & 1.403 & $1(0)$ \\
\hline & INT-across2 & $0.003(0.017)$ & $0.534(0.012)$ & $0.551(0.001)$ & $0.285(0.013)$ & 2.019 & $1(0)$ \\
\hline
\end{tabular}

MAR2A

*Auxiliary variable not included in imputation model

$\begin{array}{llllllll}\text { MI-derPassive } & \text { INT-within } & 1.479(0.016) & 0.511(0.011) & 0.470(0.002) & 2.448(0.051) & 17.341 & 0.001(0.001) \\ & \text { INT-across } & 1.094(0.015) & 0.476(0.011) & 0.473(0.002) & 1.422(0.036) & 10.073 & 0.107(0.010) \\ & \text { INT-across2 } & -1.037(0.024) & 0.761(0.017) & 0.671(0.003) & 1.654(0.053) & 11.717 & 0.957(0.006)\end{array}$




\section{MULTIPLE IMPUTATION IN PROPENSITY SCORE MATCHING}

\begin{tabular}{|c|c|c|c|c|c|c|c|}
\hline \multirow[t]{2}{*}{ Ml-regActive } & INT-within & $3.258(0.022)$ & $0.684(0.015)$ & $0.582(0.007)$ & $11.081(0.128)$ & 78.497 & $0(0)$ \\
\hline & INT-across & $2.252(0.050)$ & $1.587(0.036)$ & $1.769(0.048)$ & $7.587(0.663)$ & 53.746 & $0.539(0.016)$ \\
\hline \multirow[t]{3}{*}{ MI-redActive } & INT-within & $3.433(0.025)$ & $0.781(0.017)$ & $0.605(0.008)$ & $12.397(0.181)$ & 87.819 & $0(0)$ \\
\hline & INT-across & $2.001(0.026)$ & $0.818(0.018)$ & $0.805(0.009)$ & $4.674(0.146)$ & 33.110 & $0.198(0.013)$ \\
\hline & INT-across2 & $-0.950(0.024)$ & $0.756(0.017)$ & $0.654(0.002)$ & $1.473(0.049)$ & 10.435 & $1(0)$ \\
\hline \multicolumn{8}{|c|}{ Auxiliary variable included in imputation model } \\
\hline \multirow[t]{3}{*}{ MI-derPassive } & INT-within & $0.319(0.012)$ & $0.366(0.008)$ & $0.378(0.001)$ & $0.236(0.009)$ & 1.672 & $1(0)$ \\
\hline & INT-across & $0.039(0.012)$ & $0.379(0.008)$ & $0.380(0.001)$ & $0.145(0.006)$ & 1.027 & $1(0)$ \\
\hline & INT-across2 & $-1.104(0.024)$ & $0.756(0.017)$ & $0.677(0.003)$ & $1.789(0.057)$ & 12.673 & $0.954(0.007)$ \\
\hline \multirow[t]{2}{*}{ MI-regActive } & INT-within & $0.419(0.012)$ & $0.394(0.009)$ & $0.406(0.001)$ & $0.331(0.012)$ & 2.345 & $1(0)$ \\
\hline & INT-across & $1.642(0.120)$ & $3.807(0.085)$ & $3.516(0.057)$ & $17.172(1.684)$ & 121.645 & $0.991(0.003)$ \\
\hline \multirow[t]{3}{*}{ Ml-redActive } & INT-within & $0.418(0.012)$ & $0.381(0.009)$ & $0.389(0.001)$ & $0.320(0.011)$ & 2.267 & $1(0)$ \\
\hline & INT-across & $0.045(0.012)$ & $0.383(0.009)$ & $0.388(0.001)$ & $0.149(0.007)$ & 1.056 & $1(0)$ \\
\hline & INT-across2 & $-1.073(0.023)$ & $0.741(0.017)$ & $0.682(0.002)$ & $1.700(0.056)$ & 12.043 & $0.995(0.002)$ \\
\hline
\end{tabular}

\section{MNAR}

Auxiliary variable not included in imputation model

\begin{tabular}{|c|c|c|c|c|c|c|c|}
\hline \multirow[t]{3}{*}{ MI-derPassive } & INT-within & $1.487(0.016)$ & $0.515(0.012)$ & $0.470(0.002)$ & $2.476(0.052)$ & 17.540 & $0.001(0.001)$ \\
\hline & INT-across & $1.096(0.015)$ & $0.485(0.011)$ & $0.473(0.002)$ & $1.437(0.037)$ & 10.180 & $0.103(0.01)$ \\
\hline & INT-across2 & $-1.345(0.021)$ & $0.675(0.015)$ & $0.658(0.003)$ & $2.264(0.06)$ & 16.038 & $0.332(0.015)$ \\
\hline \multirow[t]{2}{*}{ MI-regActive } & INT-within & $3.384(0.016)$ & $0.498(0.011)$ & $0.541(0.004)$ & $11.701(0.111)$ & 82.889 & $0(0)$ \\
\hline & INT-across & $2.371(0.062)$ & $1.953(0.044)$ & $2.255(0.050)$ & $9.430(0.793)$ & 66.801 & $0.666(0.015)$ \\
\hline \multirow[t]{3}{*}{ Ml-redActive } & INT-within & $3.404(0.016)$ & $0.519(0.012)$ & $0.539(0.007)$ & $11.860(0.119)$ & 84.015 & $0(0)$ \\
\hline & INT-across & $1.878(0.021)$ & $0.661(0.015)$ & $0.719(0.007)$ & $3.963(0.092)$ & 28.074 & $0.1(0.009)$ \\
\hline & INT-across2 & $-1.261(0.02)$ & $0.637(0.014)$ & $0.646(0.002)$ & $1.996(0.054)$ & 14.139 & $0.483(0.016)$ \\
\hline \multicolumn{8}{|c|}{ Auxiliary variable included in imputation model } \\
\hline \multirow[t]{3}{*}{ Ml-derPassive } & INT-within & $0.408(0.012)$ & $0.377(0.008)$ & $0.380(0.001)$ & $0.309(0.011)$ & 2.189 & $1(0)$ \\
\hline & INT-across & $0.042(0.012)$ & $0.382(0.009)$ & $0.378(0.001)$ & $0.148(0.007)$ & 1.048 & $1(0)$ \\
\hline & INT-across2 & $-1.367(0.021)$ & $0.679(0.015)$ & $0.667(0.003)$ & $2.33(0.062)$ & 16.506 & $0.323(0.015)$ \\
\hline Ml-regActive & INT-within & $0.490(0.013)$ & $0.398(0.009)$ & $0.406(0.001)$ & $0.398(0.015)$ & 2.819 & $1(0)$ \\
\hline
\end{tabular}




\section{LING ET AL.}

\begin{tabular}{llllllcr} 
& INT-across & $2.505(0.159)$ & $5.024(0.112)$ & $4.295(0.054)$ & $31.492(2.419)$ & 223.087 & $0.957(0.006)$ \\
MI-redActive & INT-within & $0.480(0.012)$ & $0.387(0.009)$ & $0.392(0.001)$ & $0.380(0.014)$ & 2.692 & $1(0)$ \\
& INT-across & $0.045(0.013)$ & $0.396(0.009)$ & $0.389(0.001)$ & $0.159(0.007)$ & 1.126 & $1(0)$ \\
& INT-across2 & $-1.325(0.021)$ & $0.661(0.015)$ & $0.673(0.002)$ & $2.191(0.058)$ & 15.521 & $0.455(0.016)$ \\
\hline
\end{tabular}

*Note that since missingness in MAR2A is associated with an auxiliary variable, when the auxiliary variable was not included in the imputation, the imputation model is misspecified and the missing data mechanism becomes an MNAR scenario. 


\section{MULTIPLE IMPUTATION IN PROPENSITY SCORE MATCHING}

Table A5. Main simulation results: bias, standard error mean squared error (MSE), relative mean squared error (rMSE), and coverage calculated using MI-regPassive and MI-derPassive when $X_{2}$ was missing MAR2B and $Z_{p s}$ was missing under various missing data mechanisms (aux_MCAR, aux_MAR1, and aux_MAR2). Monte Carlo standard errors are in parentheses.

\begin{tabular}{|c|c|c|c|c|c|c|c|}
\hline \multicolumn{2}{|c|}{ MI Strategy } & \multirow[b]{2}{*}{ Bias } & \multicolumn{2}{|c|}{ Standard Error } & \multirow[b]{2}{*}{ MSE } & \multirow[b]{2}{*}{ rMSE } & \multirow[b]{2}{*}{ Coverage } \\
\hline Imputation & Integration & & Empirical & Bootstrap & & & \\
\hline \multicolumn{8}{|c|}{ Fully observed aux } \\
\hline \multirow[t]{3}{*}{ MI-derPassive } & INT-within & $-0.019(0.012)$ & $0.381(0.009)$ & $0.378(0.001)$ & $0.146(0.006)$ & 1.032 & $1(0)$ \\
\hline & INT-across & $-0.205(0.012)$ & $0.394(0.009)$ & $0.381(0.001)$ & $0.197(0.008)$ & 1.394 & $1(0)$ \\
\hline & INT-across2 & $-1.016(0.021)$ & $0.652(0.015)$ & $0.682(0.003)$ & $1.457(0.048)$ & 10.319 & $0.993(0.003)$ \\
\hline
\end{tabular}

aux_MCAR

Default imputation order

$\begin{array}{llllllrr}\text { MI-derPassive } & \text { INT-within } & 0.085(0.012) & 0.381(0.009) & 0.550(0.006) & 0.152(0.007) & 1.079 & 1(0) \\ & \text { INT-across } & -0.152(0.013) & 0.402(0.009) & 0.499(0.004) & 0.184(0.008) & 1.304 & 1(0) \\ & \text { INT-across2 } & -0.982(0.021) & 0.671(0.015) & 0.683(0.002) & 1.415(0.048) & 10.024 & 1(0) \\ \text { MI-regPassive } & \text { INT-within } & 0.087(0.012) & 0.378(0.008) & 0.533(0.006) & 0.150(0.007) & 1.065 & 1(0) \\ & \text { INT-across } & -0.143(0.013) & 0.413(0.009) & 0.489(0.003) & 0.191(0.008) & 1.352 & 1(0) \\ & \text { INT-across2 } & -0.996(0.021) & 0.666(0.015) & 0.685(0.002) & 1.436(0.046) & 10.173 & 1(0)\end{array}$

Reverse imputation order

\begin{tabular}{clllllrr} 
MI-derPassive & INT-within & $0.028(0.012)$ & $0.382(0.009)$ & $0.550(0.006)$ & $0.147(0.006)$ & 1.040 & $1(0)$ \\
& INT-across & $-0.191(0.012)$ & $0.391(0.009)$ & $0.500(0.004)$ & $0.189(0.008)$ & 1.340 & $1(0)$ \\
& INT-across2 & $-1.01(0.021)$ & $0.671(0.015)$ & $0.682(0.002)$ & $1.471(0.047)$ & 10.418 & $0.999(0.001)$ \\
MI-regPassive & INT-within & $-0.196(0.012)$ & $0.372(0.008)$ & $0.532(0.006)$ & $0.177(0.008)$ & 1.251 & $1(0)$ \\
& INT-across & $-0.232(0.013)$ & $0.405(0.009)$ & $0.489(0.003)$ & $0.218(0.009)$ & 1.543 & $1(0)$ \\
& INT-across2 & $-1.002(0.021)$ & $0.668(0.015)$ & $0.684(0.002)$ & $1.450(0.045)$ & 10.273 & $1(0)$ \\
\hline
\end{tabular}

aux_MAR1

Default imputation order

$\begin{array}{llllllr}\text { MI-derPassive } & \text { INT-within } & 0.185(0.012) & 0.393(0.009) & 0.550(0.006) & 0.189(0.008) & 1.337 \\ & \text { INT-across } & 0.011(0.013) & 0.414(0.009) & 0.499(0.004) & 0.171(0.008) & 1.211 \\ & \text { INT-across2 } & -1.017(0.021) & 0.651(0.015) & 0.684(0.002) & 1.458(0.045) & 10.329\end{array}$




\section{LING ET AL.}

\begin{tabular}{|c|c|c|c|c|c|c|c|}
\hline \multirow[t]{3}{*}{ MI-regPassive } & INT-within & $0.205(0.013)$ & $0.396(0.009)$ & $0.533(0.006)$ & $0.199(0.008)$ & 1.410 & $1(0)$ \\
\hline & INT-across & $0.014(0.013)$ & $0.414(0.009)$ & $0.488(0.003)$ & $0.171(0.008)$ & 1.213 & $1(0)$ \\
\hline & INT-across2 & $-1.007(0.021)$ & $0.656(0.015)$ & $0.685(0.002)$ & $1.444(0.045)$ & 10.231 & $1(0)$ \\
\hline \multicolumn{8}{|c|}{ Reverse imputation order } \\
\hline \multirow[t]{3}{*}{ Ml-derPassive } & INT-within & $0.091(0.012)$ & $0.391(0.009)$ & $0.550(0.006)$ & $0.161(0.007)$ & 1.140 & $1(0)$ \\
\hline & INT-across & $-0.069(0.013)$ & $0.408(0.009)$ & $0.500(0.004)$ & $0.171(0.007)$ & 1.213 & $1(0)$ \\
\hline & INT-across2 & $-0.993(0.021)$ & $0.661(0.015)$ & $0.684(0.002)$ & $1.422(0.045)$ & 10.071 & $1(0)$ \\
\hline \multirow[t]{3}{*}{ MI-regPassive } & INT-within & $-0.129(0.012)$ & $0.379(0.008)$ & $0.534(0.006)$ & $0.160(0.007)$ & 1.135 & $1(0)$ \\
\hline & INT-across & $-0.087(0.013)$ & $0.403(0.009)$ & $0.489(0.003)$ & $0.170(0.008)$ & 1.201 & $1(0)$ \\
\hline & INT-across2 & $-0.982(0.021)$ & $0.650(0.015)$ & $0.685(0.002)$ & $1.385(0.043)$ & 9.813 & $1(0)$ \\
\hline \multicolumn{8}{|l|}{ aux_MAR2 } \\
\hline \multicolumn{8}{|c|}{ Default imputation order } \\
\hline \multirow[t]{3}{*}{ MI-derPassive } & INT-within & $-0.060(0.012)$ & $0.390(0.009)$ & $0.550(0.006)$ & $0.155(0.006)$ & 1.100 & $1(0)$ \\
\hline & INT-across & $-0.374(0.013)$ & $0.412(0.009)$ & $0.500(0.004)$ & $0.310(0.012)$ & 2.194 & $1(0)$ \\
\hline & INT-across2 & $-0.994(0.021)$ & $0.660(0.015)$ & $0.683(0.002)$ & $1.422(0.044)$ & 10.076 & $1(0)$ \\
\hline \multirow[t]{3}{*}{ MI-regPassive } & INT-within & $-0.049(0.013)$ & $0.396(0.009)$ & $0.533(0.006)$ & $0.159(0.006)$ & 1.128 & $1(0)$ \\
\hline & INT-across & $-0.369(0.013)$ & $0.408(0.009)$ & $0.489(0.003)$ & $0.302(0.012)$ & 2.141 & $1(0)$ \\
\hline & INT-across2 & $-1.001(0.021)$ & $0.656(0.015)$ & $0.685(0.002)$ & $1.431(0.043)$ & 10.137 & $0.999(0.001)$ \\
\hline \multicolumn{8}{|c|}{ Reverse imputation order } \\
\hline \multirow[t]{3}{*}{ Ml-derPassive } & INT-within & $-0.068(0.012)$ & $0.390(0.009)$ & $0.549(0.006)$ & $0.157(0.006)$ & 1.111 & $1(0)$ \\
\hline & INT-across & $-0.369(0.013)$ & $0.412(0.009)$ & $0.500(0.004)$ & $0.305(0.012)$ & 2.163 & $1(0)$ \\
\hline & INT-across2 & $-0.996(0.021)$ & $0.652(0.015)$ & $0.683(0.002)$ & $1.416(0.043)$ & 10.032 & $0.999(0.001)$ \\
\hline \multirow[t]{3}{*}{ Ml-regPassive } & INT-within & $-0.308(0.012)$ & $0.391(0.009)$ & $0.533(0.006)$ & $0.248(0.010)$ & 1.754 & $1(0)$ \\
\hline & INT-across & $-0.414(0.013)$ & $0.411(0.009)$ & $0.489(0.003)$ & $0.340(0.013)$ & 2.410 & $1(0)$ \\
\hline & INT-across2 & $-0.987(0.021)$ & $0.665(0.015)$ & $0.685(0.002)$ & $1.417(0.043)$ & 10.039 & $1(0)$ \\
\hline
\end{tabular}




\section{MULTIPLE IMPUTATION IN PROPENSITY SCORE MATCHING}

Table A6. Summary statistics, missing values, and standardized differences (pre-matching and post-matching in commonly applied methods) in DIETFITS trial participants $(\mathrm{N}=609)$.

\begin{tabular}{|c|c|c|c|c|c|c|c|c|c|c|c|}
\hline \multirow[t]{3}{*}{ Variable } & \multicolumn{4}{|c|}{ Summary Statistics } & \multicolumn{7}{|c|}{ Standardized Difference } \\
\hline & \multicolumn{2}{|c|}{ Intervention (N=97) } & \multicolumn{2}{|l|}{ Control (N=512) } & \multirow{2}{*}{$\begin{array}{c}\text { Pre- } \\
\text { matching }\end{array}$} & \multicolumn{2}{|c|}{ Complete Case Analysis } & \multirow{2}{*}{$\begin{array}{c}\text { Mean } \\
\text { Imputation } \\
\text { original }\end{array}$} & \multicolumn{2}{|c|}{ Mean Imputation } & \multirow{2}{*}{$\begin{array}{c}\text { Missing } \\
\text { Indicator } \\
\text { original }\end{array}$} \\
\hline & Summary & Missing & Summary & Missing & & original & complete cases & & original & imputed & \\
\hline Age (years) & $39.38 \pm 6.06$ & $\begin{array}{c}5 \\
(5.15 \%)\end{array}$ & $41.23 \pm 6.78$ & $\begin{array}{c}127 \\
(24.80 \%)\end{array}$ & -0.29 & -0.4 & 0.08 & - & 0.04 & 0.01 & 0.11 \\
\hline Sex (female) & $62(63.92 \%)$ & 0 & $284(55.47 \%)$ & 0 & 0.17 & -0.21 & 0.02 & 0 & 0.11 & 0.11 & -0.11 \\
\hline Race & & $\begin{array}{c}1 \\
(1.03 \%)\end{array}$ & & $5(0.98 \%)$ & - & & & & & & \\
\hline Caucasian & $52(53.61 \%)$ & - & $302(58.98 \%)$ & - & -0.11 & -0.23 & 0.07 & - & -0.23 & 0.21 & -0.25 \\
\hline Hispanic & $27(27.84 \%)$ & - & $103(20.12 \%)$ & - & 0.18 & 0.16 & 0 & - & 0.22 & -0.02 & 0.19 \\
\hline Black & $3(3.09 \%)$ & - & $20(3.91 \%)$ & - & -0.04 & 0.22 & 0.09 & - & -0.06 & -0.15 & -0.1 \\
\hline $\begin{array}{c}\text { Asian/Pacific } \\
\text { Islander }\end{array}$ & $7(7.22 \%)$ & - & $57(11.13 \%)$ & - & -0.14 & -0.11 & -0.15 & - & -0.07 & -0.21 & 0.03 \\
\hline Other & $7(7.22 \%)$ & - & $25(4.88 \%)$ & - & 0.1 & 0.14 & 0 & - & 0 & 0 & 0 \\
\hline $\begin{array}{c}\text { Weight }(\mathrm{kg}) \\
\text { change } 6 \\
\text { months } \\
\end{array}$ & $-6.19 \pm 5.19$ & $\begin{array}{c}8 \\
(8.25 \%)\end{array}$ & $-7.45 \pm 6.11$ & $\begin{array}{c}135 \\
(26.37 \%)\end{array}$ & 0.22 & 0.38 & -0.02 & - & -0.02 & 0 & -0.11 \\
\hline $\begin{array}{c}\text { Class } \\
\text { attendance } 6 \\
\text { months (\%) }\end{array}$ & $76.86 \pm 17.01$ & 0 & $72.88 \pm 22.47$ & 0 & 0.2 & -0.17 & -0.06 & 0 & -0.19 & -0.19 & -0.06 \\
\hline
\end{tabular}

*post-matching standardized differences were measured both in the original dataset with missingness and the analytical datasets (e.g. complete cases for complete cases analysis and imputed dataset for mean imputation) 


\section{LING ET AL.}

Table A7. Post-matching standardized differences for all multiple imputation (MI) methods in DIETFITS trial participants (N=609). Note that those whose absolute values are above 0.1 are bolded.

\begin{tabular}{|c|c|c|c|c|c|c|c|c|c|c|}
\hline \multirow[b]{2}{*}{ MI Method } & \multirow[b]{2}{*}{ Dataset } & \multirow{2}{*}{$\begin{array}{c}\text { Age } \\
\text { (years) }\end{array}$} & \multirow{2}{*}{$\begin{array}{c}\text { Sex } \\
\text { (female) }\end{array}$} & \multicolumn{5}{|c|}{ Race } & \multirow{2}{*}{$\begin{array}{c}\text { Weight (kg) } \\
\text { change } 6 \\
\text { months }\end{array}$} & \multirow{2}{*}{$\begin{array}{c}\text { Class } \\
\text { attendance } \\
6 \text { months } \\
(\%)\end{array}$} \\
\hline & & & & Caucasian & Hispanic & Black & Islander & Other & & \\
\hline \multicolumn{11}{|c|}{ Auxiliary variable not included in imputation model } \\
\hline \multirow[t]{2}{*}{ derPassive-within } & original & -0.02 & 0.03 & -0.31 & 0.21 & 0.04 & 0.02 & 0.02 & 0.11 & 0 \\
\hline & imputed & 0.02 & 0.03 & 0.04 & -0.01 & -0.01 & -0.01 & 0 & 0.01 & 0 \\
\hline \multirow[t]{2}{*}{ derPassive-across } & original & 0.19 & -0.16 & -0.23 & 0.12 & 0 & 0.07 & -0.04 & 0.07 & 0.13 \\
\hline & imputed & 0.17 & -0.16 & -0.06 & 0.02 & -0.06 & 0 & 0.13 & -0.05 & 0.13 \\
\hline \multirow[t]{2}{*}{ derPassive-across2 } & original & 0.09 & -0.09 & -0.38 & 0.3 & 0.07 & -0.07 & 0.1 & 0.13 & 0.09 \\
\hline & imputed & 0.07 & -0.09 & -0.08 & -0.05 & 0.07 & 0.09 & 0.13 & 0.04 & 0.09 \\
\hline \multirow[t]{2}{*}{ regActive-within } & original & -0.02 & 0.12 & -0.31 & 0.31 & -0.02 & 0.03 & -0.09 & 0.04 & 0.37 \\
\hline & imputed & -0.04 & 0.12 & 0.05 & -0.01 & 0.04 & -0.13 & 0.05 & -0.03 & 0.37 \\
\hline \multirow[t]{2}{*}{ regActive-across } & original & 0.07 & 0.02 & -0.17 & 0.24 & -0.06 & 0 & -0.19 & 0.3 & 0.28 \\
\hline & imputed & 0.07 & 0.02 & 0.23 & -0.05 & -0.1 & -0.29 & 0.09 & 0.08 & 0.28 \\
\hline \multirow[t]{2}{*}{ regActive-across2 } & original & 0.03 & 0.06 & -0.32 & 0.22 & 0.26 & 0 & -0.09 & 0.03 & 0.02 \\
\hline & imputed & 0.03 & 0.06 & 0.06 & -0.05 & 0.15 & -0.08 & -0.04 & 0.03 & 0.02 \\
\hline \multirow[t]{2}{*}{ redActive-within } & original & -0.08 & 0.03 & -0.31 & 0.24 & -0.03 & 0.06 & -0.04 & 0.09 & -0.02 \\
\hline & imputed & 0 & 0.03 & 0.02 & -0.03 & -0.01 & -0.01 & 0.05 & 0 & -0.02 \\
\hline \multirow[t]{2}{*}{ redActive-across } & original & 0.08 & 0.19 & -0.28 & 0.33 & 0 & 0 & -0.2 & 0.2 & -0.09 \\
\hline & imputed & 0.06 & 0.19 & 0 & -0.05 & 0 & 0.15 & 0 & 0.19 & -0.09 \\
\hline \multirow[t]{2}{*}{ redActive-across2 } & original & 0.03 & 0.06 & -0.32 & 0.22 & 0.26 & 0 & -0.09 & 0.03 & 0.02 \\
\hline & imputed & 0.03 & 0.06 & 0.06 & -0.05 & 0.15 & -0.08 & -0.04 & 0.03 & 0.02 \\
\hline \multicolumn{11}{|c|}{ Auxiliary variable included in imputation model } \\
\hline \multirow[t]{2}{*}{ derPassive-within } & original & -0.1 & -0.01 & -0.29 & 0.2 & -0.02 & 0.04 & 0.02 & -0.11 & -0.02 \\
\hline & imputed & -0.07 & -0.01 & -0.07 & 0.08 & 0 & -0.01 & 0.03 & -0.06 & -0.02 \\
\hline \multirow[t]{2}{*}{ derPassive-across } & original & -0.14 & 0.02 & -0.29 & 0.27 & -0.1 & -0.03 & 0.05 & -0.06 & -0.11 \\
\hline & imputed & -0.12 & 0.02 & -0.02 & 0.09 & 0 & 0.09 & -0.17 & -0.01 & -0.11 \\
\hline
\end{tabular}


MULTIPLE IMPUTATION IN PROPENSITY SCORE MATCHING

\begin{tabular}{|c|c|c|c|c|c|c|c|c|c|c|}
\hline \multirow[t]{2}{*}{ derPassive-across2 } & original & -0.23 & -0.02 & -0.27 & 0.24 & -0.15 & -0.03 & 0.1 & -0.18 & -0.06 \\
\hline & imputed & -0.2 & -0.02 & 0.02 & 0.05 & -0.1 & 0.09 & -0.11 & -0.05 & -0.06 \\
\hline \multirow[t]{2}{*}{ regActive-within } & original & -0.17 & -0.01 & -0.33 & 0.23 & -0.04 & 0.05 & 0.05 & -0.04 & 0.34 \\
\hline & imputed & -0.11 & -0.01 & 0.01 & -0.13 & 0.07 & 0.15 & 0.03 & 0.37 & 0.34 \\
\hline \multirow[t]{2}{*}{ regActive-across } & original & -0.11 & -0.07 & -0.25 & 0.05 & -0.06 & 0.15 & 0.1 & 0.02 & 0.32 \\
\hline & imputed & -0.13 & -0.07 & -0.02 & -0.16 & 0.07 & 0.17 & 0.13 & 0.33 & 0.32 \\
\hline \multirow[t]{2}{*}{ regActive-across2 } & original & -0.12 & -0.11 & -0.4 & 0.21 & 0.15 & 0.11 & 0 & -0.01 & -0.06 \\
\hline & imputed & -0.09 & -0.11 & -0.19 & 0.14 & 0.15 & 0 & 0.04 & -0.06 & -0.06 \\
\hline \multirow[t]{2}{*}{ redActive-within } & original & -0.07 & -0.01 & -0.29 & 0.19 & 0.01 & 0.03 & -0.01 & -0.08 & -0.07 \\
\hline & imputed & -0.04 & -0.01 & -0.07 & 0.09 & 0.02 & 0 & 0 & -0.04 & -0.07 \\
\hline \multirow[t]{2}{*}{ redActive-across } & original & -0.08 & -0.13 & -0.36 & 0.12 & 0.15 & 0.19 & 0 & -0.06 & -0.06 \\
\hline & imputed & -0.08 & -0.13 & -0.15 & 0.14 & 0.07 & -0.04 & 0.04 & -0.07 & -0.06 \\
\hline \multirow[t]{2}{*}{ redActive-across2 } & original & -0.12 & -0.11 & -0.4 & 0.21 & 0.15 & 0.11 & 0 & -0.01 & -0.06 \\
\hline & imputed & -0.09 & -0.11 & -0.19 & 0.14 & 0.15 & 0 & 0.04 & -0.06 & -0.06 \\
\hline
\end{tabular}




\section{LING ET AL.}

Table A8. Sensitivity simulation results (with reduced confounding effects): balance diagnosis and treatment effect estimation results using commonly applied missing data method before propensity score matching (PSM), in reference to applying PSM to full data without missingness (Monte Carlo standard errors in parentheses). MDM=missing data mechanism, $\mathrm{CC}=$ complete-case analysis, CVA = complete-variable analysis.

\begin{tabular}{|c|c|c|c|c|c|c|c|c|c|c|c|}
\hline \multirow[b]{2}{*}{ MDM } & \multirow[b]{2}{*}{ Method } & \multirow[b]{2}{*}{ Bias } & \multicolumn{2}{|c|}{ Standard Error } & \multirow[b]{2}{*}{ MSE } & \multirow[b]{2}{*}{ rMSE } & \multirow[b]{2}{*}{ Coverage } & \multirow[b]{2}{*}{$\begin{array}{r}\text { Percentage } \\
\text { Matched }\end{array}$} & \multicolumn{3}{|c|}{$\begin{array}{c}\text { Post-Matching Standardized } \\
\text { Difference }\end{array}$} \\
\hline & & & Empirical & Robust & & & & & $\begin{array}{l}\text { Underlying } \\
\text { data }\end{array}$ & $\mathrm{X} 1$ & $\mathrm{X} 2$ \\
\hline \multicolumn{12}{|c|}{ ำ } \\
\hline Data & NA & 0.001 & 0.298 & 0.310 & 0.089 & 1.000 & 0.960 & 1.000 & - & 0 & 0 \\
\hline \multirow{10}{*}{ MCAR } & & -0.009 & 0.429 & 0.439 & 0.184 & & 0.955 & & & & \\
\hline & $\mathrm{CC}$ & $(0.014)$ & $(0.01)$ & $(0.001)$ & $(0.008)$ & 2.076 & $(0.007)$ & 0.999 & - & 0 & 0 \\
\hline & & 1.732 & 0.44 & 0.435 & 3.194 & & 0.028 & & & & \\
\hline & CVA & $(0.014)$ & $(0.01)$ & $(0.001)$ & $(0.048)$ & 35.975 & $(0.005)$ & 1 & - & 0 & 0.313 \\
\hline & Mean & 0.855 & 0.377 & 0.378 & 0.873 & & 0.387 & & & & \\
\hline & Imputation & $(0.012)$ & $(0.008)$ & $(0)$ & $(0.021)$ & 9.838 & $(0.015)$ & 1 & original data & 0 & 0.156 \\
\hline & & & & & & & & & imputed data & 0 & 0 \\
\hline & $\begin{array}{l}\text { Missing } \\
\text { Indicator }\end{array}$ & $\begin{array}{r}0.858 \\
(0.012)\end{array}$ & $\begin{array}{r}0.373 \\
(0.008)\end{array}$ & $\begin{array}{r}0.379 \\
(0)\end{array}$ & $\begin{array}{r}0.876 \\
(0.021)\end{array}$ & 9.867 & $\begin{array}{r}0.383 \\
(0.015)\end{array}$ & 1 & full data & 0 & 0.157 \\
\hline & & & & & & & & & observed part & 0 & 0.14 \\
\hline & & & & & & & & & missing part & NA & 0.138 \\
\hline \multirow{10}{*}{ MAR1 } & & -1.69 & 0.632 & 0.649 & 3.256 & & 0.269 & & & & \\
\hline & CC & $(0.02)$ & $(0.014)$ & $(0.002)$ & $(0.07)$ & 36.672 & $(0.014)$ & 1 & - & 0 & 0 \\
\hline & & 1.729 & 0.439 & 0.434 & 3.181 & & 0.023 & & & & \\
\hline & CVA & $(0.014)$ & $(0.01)$ & $(0.001)$ & $(0.048)$ & 35.831 & $(0.005)$ & 1 & - & 0 & 0.314 \\
\hline & Mean & 2.763 & 0.477 & 0.484 & 7.863 & & & & & & \\
\hline & Imputation & $(0.015)$ & $(0.011)$ & $(0.001)$ & $(0.084)$ & 88.566 & $0(0)$ & 0.722 & original data & -0.003 & 0.483 \\
\hline & & & & & & & & & imputed data & -0.003 & -0.003 \\
\hline & $\begin{array}{l}\text { Missing } \\
\text { Indicator }\end{array}$ & $\begin{array}{r}2.75 \\
(0.015)\end{array}$ & $\begin{array}{r}0.477 \\
(0.011)\end{array}$ & $\begin{array}{r}0.484 \\
(0.001)\end{array}$ & $\begin{array}{r}7.792 \\
(0.084)\end{array}$ & 87.769 & $0(0)$ & 0.722 & full data & -0.001 & 0.485 \\
\hline & & & & & & & & & observed part & -0.002 & 0.26 \\
\hline & & & & & & & & & missing part & NA & 0.26 \\
\hline \multirow{9}{*}{ MAR2A } & & -0.679 & 0.529 & 0.531 & 0.741 & & 0.751 & & & & \\
\hline & CC & $(0.017)$ & $(0.012)$ & $(0.001)$ & $(0.027)$ & 8.349 & $(0.014)$ & 1 & - & 0.001 & 0 \\
\hline & & 1.74 & 0.431 & 0.434 & 3.213 & & 0.029 & & & & \\
\hline & CVA & $(0.014)$ & $(0.01)$ & $(0.001)$ & $(0.047)$ & 36.187 & $(0.005)$ & 1 & - & 0 & 0.314 \\
\hline & Mean & 3.203 & 0.446 & 0.443 & 10.457 & & & & & & \\
\hline & Imputation & $(0.014)$ & $(0.01)$ & $(0.001)$ & $(0.092)$ & 117.786 & $0(0)$ & 0.803 & original data & -0.001 & 0.57 \\
\hline & & & & & & & & & imputed data & -0.001 & -0.001 \\
\hline & $\begin{array}{l}\text { Missing } \\
\text { Indicator }\end{array}$ & $\begin{array}{r}3.208 \\
(0.014)\end{array}$ & $\begin{array}{l}0.437 \\
(0.01)\end{array}$ & $\begin{array}{r}0.444 \\
(0.001)\end{array}$ & $\begin{array}{l}10.484 \\
(0.089)\end{array}$ & 118.086 & $0(0)$ & 0.803 & full data & 0 & 0.571 \\
\hline & & & & & & & & & observed part & 0 & 0.312 \\
\hline
\end{tabular}


MULTIPLE IMPUTATION IN PROPENSITY SCORE MATCHING

\begin{tabular}{|c|c|c|c|c|c|c|c|c|c|c|c|}
\hline & & & & & & & & & missing part & NA & 0.312 \\
\hline \multirow{11}{*}{ MNAR } & & -0.778 & 0.527 & 0.531 & 0.882 & & 0.663 & & & & \\
\hline & CC & $(0.017)$ & $(0.012)$ & $(0.001)$ & $(0.028)$ & 9.94 & $(0.015)$ & 1 & - & 0 & 0 \\
\hline & & 1.739 & 0.43 & 0.434 & 3.209 & & 0.023 & & & & \\
\hline & CVA & $(0.014)$ & $(0.01)$ & $(0.001)$ & $(0.047)$ & 36.143 & $(0.005)$ & 1 & - & 0 & 0.314 \\
\hline & Mean & 3.213 & 0.453 & 0.446 & 10.528 & & & & & & \\
\hline & Imputation & $(0.014)$ & $(0.01)$ & $(0.001)$ & $(0.092)$ & 118.59 & $0(0)$ & & original data & 0 & 0.573 \\
\hline & & & & & & & & 0.797 & imputed data & 0 & 0 \\
\hline & Missing & 3.214 & 0.445 & 0.446 & 10.526 & & & & & & \\
\hline & Indicator & $(0.014)$ & $(0.01)$ & $(0.001)$ & $(0.091)$ & 118.57 & $0(0)$ & 0.797 & full data & 0 & 0.573 \\
\hline & & & & & & & & & observed part & 0 & 0.312 \\
\hline & & & & & & & & & missing part & NA & 0.31 \\
\hline
\end{tabular}

*The percentage of treated patients being matched using $\mathrm{CC}$ is calculated from complete cases. 


\section{LING ET AL.}

Table A9. Sensitivity simulation results (with reduced confounding effects): percentage of treated subjects matched and standardized difference of covariates after matching in various multiple imputation (MI) strategies. For INT-across and INTacross2, standardized differences were also calculated on the observed and missing part of the imputed data separately. MDM=missing data mechanism.

\begin{tabular}{|c|c|c|c|c|c|c|c|c|c|c|c|}
\hline \multirow[b]{3}{*}{ MDM } & & & \multirow[b]{3}{*}{$\begin{array}{r}\text { Percentage } \\
\text { Matched }\end{array}$} & \multicolumn{8}{|c|}{ Post-matching standardized difference } \\
\hline & \multicolumn{2}{|c|}{ MI Strategy } & & \multicolumn{2}{|c|}{ Full Data } & \multicolumn{2}{|c|}{ Imputed Data } & \multicolumn{2}{|c|}{ Observed Part } & \multicolumn{2}{|c|}{ Missing Part } \\
\hline & Imputation & Integration & & $\mathbf{X 1}$ & $\mathbf{X 2}$ & $\mathbf{X 1}$ & $\mathbf{X 2}$ & $\mathbf{X 1}$ & $\mathbf{X 2}$ & $\mathrm{X} 1$ & $\mathbf{X 2}$ \\
\hline \multicolumn{12}{|c|}{ MCAR } \\
\hline \multicolumn{12}{|c|}{ Auxiliary variable not included in imputation model } \\
\hline & \multirow[t]{3}{*}{ MI-derPassive } & INT-within & 0.652 & 0 & 0.126 & 0 & 0 & - & - & - & - \\
\hline & & INT-across & 0.633 & -0.002 & 0.008 & -0.002 & 0.006 & 0.036 & -0.114 & -0.046 & 0.125 \\
\hline & & INT-across2 & 0.636 & -0.002 & 0.012 & -0.002 & 0.01 & 0.039 & -0.108 & -0.048 & 0.127 \\
\hline & \multirow[t]{2}{*}{ MI-regActive } & INT-within & 0.791 & 0.128 & 0.6 & 0.128 & -0.127 & - & - & - & - \\
\hline & & INT-across & 0.722 & -0.004 & 0.345 & -0.004 & 0.01 & 0.01 & 0.003 & -0.019 & 0.022 \\
\hline & \multirow[t]{3}{*}{ MI-redActive } & INT-within & 0.792 & 0 & 0.656 & 0 & 0 & - & - & - & - \\
\hline & & INT-across & 0.738 & 0 & 0.345 & 0 & 0.009 & 0.004 & -0.002 & -0.004 & 0.021 \\
\hline & & INT-across2 & 0.782 & 0 & 0.43 & 0 & 0.045 & 0.001 & 0.001 & -0.002 & 0.079 \\
\hline \multicolumn{12}{|c|}{ Auxiliary variable included in imputation model } \\
\hline & \multirow[t]{3}{*}{ MI-derPassive } & INT-within & 0.655 & 0 & 0.024 & 0 & 0 & - & - & - & - \\
\hline & & INT-across & 0.652 & 0.006 & -0.008 & 0.006 & -0.001 & -0.115 & -0.088 & 0.098 & 0.066 \\
\hline & & INT-across2 & 0.653 & 0.01 & -0.011 & 0.01 & -0.003 & -0.123 & -0.087 & 0.11 & 0.062 \\
\hline & \multirow[t]{2}{*}{ MI-regActive } & INT-within & 0.659 & 0.006 & 0.04 & 0.006 & -0.011 & - & - & - & - \\
\hline & & INT-across & 0.653 & -0.028 & 0.025 & -0.028 & 0.01 & -0.122 & 0.053 & 0.049 & -0.039 \\
\hline & \multirow[t]{3}{*}{ MI-redActive } & INT-within & 0.659 & 0 & 0.051 & 0 & 0 & - & - & - & - \\
\hline & & INT-across & 0.653 & -0.031 & 0.029 & -0.031 & 0.014 & -0.203 & 0.086 & 0.11 & -0.059 \\
\hline & & INT-across2 & 0.652 & -0.043 & 0.04 & -0.043 & 0.026 & -0.214 & 0.112 & 0.1 & -0.061 \\
\hline
\end{tabular}

Auxiliary variable not included in imputation model
MI-derPassive
INT-within
0.656

0.114

0

0 


\section{MULTIPLE IMPUTATION IN PROPENSITY SCORE MATCHING}

\begin{tabular}{|c|c|c|c|c|c|c|c|c|c|c|}
\hline & INT-across & 0.617 & 0.002 & -0.028 & 0.002 & -0.015 & -0.748 & -1.105 & 0.205 & 0.647 \\
\hline & INT-across2 & 0.621 & 0.008 & -0.034 & 0.008 & -0.021 & -0.746 & -1.109 & 0.201 & 0.645 \\
\hline \multirow[t]{2}{*}{ MI-regActive } & INT-within & 0.877 & 0.368 & 0.752 & 0.368 & 0.322 & - & - & - & - \\
\hline & INT-across & 0.666 & 0.562 & 0.531 & 0.562 & 0.344 & -0.16 & -0.397 & 0.714 & 0.708 \\
\hline \multirow[b]{3}{*}{ Auxiliary variable include } & INT-within & 0.772 & 0 & 0.483 & 0 & -0.006 & - & - & - & - \\
\hline & INT-across & 0.628 & 0.002 & 0.031 & 0.002 & -0.178 & -0.746 & -1.013 & 0.241 & 0.466 \\
\hline & $\begin{array}{l}\text { INT-across2 } \\
\text { imputation model }\end{array}$ & 0.67 & 0.003 & 0.219 & 0.003 & -0.029 & -0.78 & -0.84 & 0.26 & 0.517 \\
\hline \multirow[t]{3}{*}{ MI-derPassive } & INT-within & 0.666 & 0 & 0.065 & 0 & -0.001 & - & - & - & - \\
\hline & INT-across & 0.647 & 0.026 & -0.031 & 0.026 & -0.069 & -0.895 & -1.01 & 0.467 & 0.404 \\
\hline & INT-across2 & 0.652 & 0.022 & -0.027 & 0.022 & -0.06 & -0.953 & -0.974 & 0.519 & 0.375 \\
\hline \multirow[t]{2}{*}{ MI-regActive } & INT-within & 0.785 & 0.351 & 0.42 & 0.351 & 0.338 & - & - & - & - \\
\hline & INT-across & 0.745 & 0.386 & 0.382 & 0.386 & 0.321 & -0.672 & -0.663 & 0.856 & 0.741 \\
\hline \multirow[t]{3}{*}{ MI-redActive } & INT-within & 0.669 & 0 & 0.085 & 0 & -0.001 & - & - & - & - \\
\hline & INT-across & 0.643 & 0.012 & -0.015 & 0.012 & -0.069 & -0.921 & -1.008 & 0.46 & 0.405 \\
\hline & INT-across2 & 0.651 & 0.013 & -0.016 & 0.013 & -0.061 & -0.972 & -0.982 & 0.506 & 0.384 \\
\hline
\end{tabular}

Auxiliary variable not included in imputation model

$\begin{array}{lll}\text { MI-derPassive } & \text { INT-within } & 0.75 \\ & \text { INT-across } & 0.68 \\ & \text { INT-across2 } & 0.695 \\ \text { MI-regActive } & \text { INT-within } & 0.881 \\ & \text { INT-across } & 0.57 \\ \text { MI-redActive } & \text { INT-within } & 0.863 \\ & \text { INT-across } & 0.73 \\ & \text { INT-across2 } & 0.78\end{array}$

$\begin{array}{rr}0 & 0.379 \\ 0 & 0.146 \\ 0 & 0.171 \\ 0.076 & 0.698 \\ 0.026 & 0.428 \\ 0 & 0.656 \\ 0 & 0.348 \\ 0 & 0.396\end{array}$

$\begin{array}{rr}0 & 0 \\ 0 & -0.017 \\ 0 & -0.016 \\ 0.076 & 0.078 \\ 0.026 & 0.021 \\ 0 & 0 \\ 0 & -0.095 \\ 0 & -0.09\end{array}$

$\begin{array}{rrrr}-0.39 & -1.182 & 0.226 & 0.558 \\ -0.392 & -1.185 & 0.212 & 0.563 \\ - & - & - & - \\ -0.038 & -0.472 & 0.02 & 0.204 \\ - & - & - & - \\ -0.331 & -0.99 & 0.107 & 0.221 \\ -0.403 & -1.061 & 0.14 & 0.245\end{array}$

Auxiliary variable included in imputation model

MI-derPassive

INT-within
INT-across




\section{LING ET AL.}

\begin{tabular}{lllrrrrrrrrr} 
& INT-across2 & 0.653 & -0.025 & 0.023 & -0.025 & -0.019 & -0.473 & -1.257 & 0.308 & 0.541 \\
MI-regActive & INT-within & 0.729 & 0.122 & 0.248 & 0.122 & 0.166 & - & - & - \\
& INT-across & 0.701 & 0.131 & 0.177 & 0.131 & 0.126 & -0.374 & -1.061 & 0.51 & 0.614 \\
\multirow{2}{*}{ MI-redActive } & INT-within & 0.672 & 0 & 0.084 & 0 & -0.001 & - & - & - \\
& INT-across & 0.652 & -0.018 & 0.017 & -0.018 & -0.032 & -0.476 & -1.23 & 0.341 & 0.48 \\
& INT-across2 & 0.652 & -0.026 & 0.024 & -0.026 & -0.023 & -0.506 & -1.203 & 0.361 & 0.465 \\
\hline
\end{tabular}

MNAR

Auxiliary variable not included in imputation model

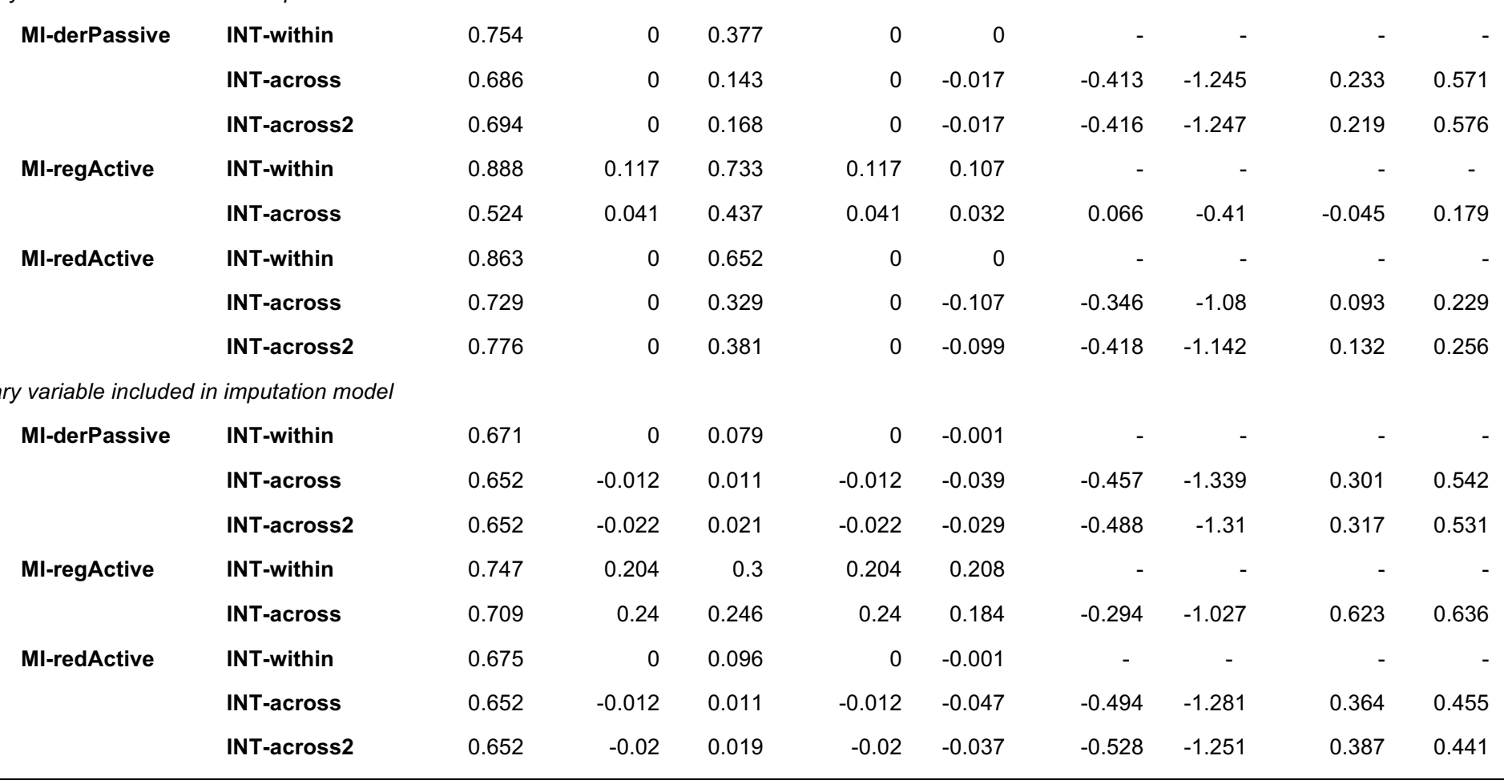




\section{MULTIPLE IMPUTATION IN PROPENSITY SCORE MATCHING}

Table A10. Sensitivity simulation results (with reduced confounding effects): bias, standard error, mean squared error (MSE), relative mean squared error (rMSE), and coverage (calculated using bootstrap standard error) results using various multiple imputation (MI) strategies in MCAR, MAR1, MAR2A, and MNAR. (Monte Carlo standard errors in parentheses).

\begin{tabular}{|c|c|c|c|c|c|c|c|}
\hline \multicolumn{2}{|c|}{ MI Strategy } & \multirow[b]{2}{*}{ Bias } & \multicolumn{2}{|c|}{ Standard Error } & \multirow[b]{2}{*}{ MSE } & \multirow[b]{2}{*}{ rMSE } & \multirow[b]{2}{*}{ Coverage } \\
\hline Imputation & Integration & & Empirical & Bootstrap & & & \\
\hline \multicolumn{8}{|l|}{ MCAR } \\
\hline \multicolumn{8}{|c|}{ Auxiliary variable not included in imputation model } \\
\hline \multirow[t]{3}{*}{ MI-derPassive } & INT-within & $-0.001(0.01)$ & $0.316(0.007)$ & $0.315(0)$ & $0.099(0.004)$ & 1.115 & $1(0)$ \\
\hline & INT-across & $-0.355(0.011)$ & $0.348(0.008)$ & $0.348(0.001)$ & $0.247(0.009)$ & 2.782 & $1(0)$ \\
\hline & INT-across2 & $0.013(0.013)$ & $0.424(0.009)$ & $0.433(0.001)$ & $0.18(0.008)$ & 2.028 & $1(0)$ \\
\hline \multirow[t]{2}{*}{ Ml-regActive } & INT-within & $0.973(0.011)$ & $0.344(0.008)$ & $0.36(0.003)$ & $1.064(0.024)$ & 11.985 & $0.042(0.006)$ \\
\hline & INT-across & $-0.423(0.014)$ & $0.45(0.01)$ & $0.548(0.002)$ & $0.381(0.014)$ & 4.292 & $1(0)$ \\
\hline \multirow[t]{3}{*}{ MI-redActive } & INT-within & $0.967(0.011)$ & $0.343(0.008)$ & $0.343(0.001)$ & $1.052(0.024)$ & 11.85 & $0.01(0.003)$ \\
\hline & INT-across & $-1.386(0.018)$ & $0.584(0.013)$ & $0.525(0.002)$ & $2.261(0.055)$ & 25.468 & $0.004(0.002)$ \\
\hline & INT-across2 & $0.015(0.014)$ & $0.44(0.01)$ & $0.436(0.001)$ & $0.194(0.009)$ & 2.185 & $1(0)$ \\
\hline \multicolumn{8}{|c|}{ Auxiliary variable included in imputation model } \\
\hline \multirow[t]{3}{*}{ MI-derPassive } & INT-within & $0.031(0.008)$ & $0.266(0.006)$ & $0.279(0)$ & $0.072(0.003)$ & 0.811 & $1(0)$ \\
\hline & INT-across & $-0.044(0.01)$ & $0.317(0.007)$ & $0.356(0.001)$ & $0.103(0.005)$ & 1.16 & $1(0)$ \\
\hline & INT-across2 & $0.004(0.014)$ & $0.458(0.01)$ & $0.444(0.001)$ & $0.209(0.01)$ & 2.354 & $1(0)$ \\
\hline \multirow[t]{2}{*}{ MI-regActive } & INT-within & $0.169(0.009)$ & $0.27(0.006)$ & $0.284(0)$ & $0.101(0.005)$ & 1.138 & $1(0)$ \\
\hline & INT-across & $0.231(0.01)$ & $0.329(0.007)$ & $0.355(0.001)$ & $0.162(0.007)$ & 1.825 & $1(0)$ \\
\hline \multirow[t]{3}{*}{ MI-redActive } & INT-within & $0.17(0.009)$ & $0.269(0.006)$ & $0.282(0)$ & $0.101(0.005)$ & 1.138 & $1(0)$ \\
\hline & INT-across & $-0.14(0.01)$ & $0.325(0.007)$ & $0.413(0.001)$ & $0.125(0.006)$ & 1.408 & $1(0)$ \\
\hline & INT-across2 & $0.013(0.014)$ & $0.456(0.01)$ & $0.448(0.001)$ & $0.208(0.01)$ & 2.343 & $1(0)$ \\
\hline
\end{tabular}

\section{MAR1}

Auxiliary variable not included in imputation model

Ml-derPassive

$\begin{array}{ll}\text { INT-within } & 0.149(0.012) \\ \text { INT-across } & 0.203(0.012) \\ \text { INT-across2 } & -2.102(0.019)\end{array}$

$0.374(0.008)$

$0.372(0.001)$

$0.162(0.007)$

1.825

$1(0)$

INT-across2

$0.385(0.009)$

$0.396(0.002)$

$0.189(0.008)$

2.129

$1(0)$

$0.609(0.014)$

0.607 (0.002)

4.791 (0.082)

53.966

$0(0)$ 


\section{LING ET AL.}

\begin{tabular}{|c|c|c|c|c|c|c|c|}
\hline \multirow[t]{2}{*}{ MI-regActive } & INT-within & $1.495(0.012)$ & $0.381(0.009)$ & $0.406(0.001)$ & $2.381(0.037)$ & 26.82 & $0(0)$ \\
\hline & INT-across & $-0.197(0.033)$ & $1.057(0.024)$ & $1.114(0.008)$ & $1.154(0.054)$ & 12.999 & $1(0)$ \\
\hline \multirow[t]{3}{*}{ MI-redActive } & INT-within & $1.496(0.012)$ & $0.385(0.009)$ & $0.397(0.001)$ & $2.386(0.038)$ & 26.876 & $0(0)$ \\
\hline & INT-across & $0.273(0.077)$ & $2.436(0.054)$ & $2.089(0.016)$ & $6.001(0.232)$ & 67.595 & $1(0)$ \\
\hline & INT-across2 & $-1.972(0.019)$ & $0.591(0.013)$ & $0.608(0.001)$ & $4.236(0.075)$ & 47.714 & $0(0)$ \\
\hline \multicolumn{8}{|c|}{ uxiliary variable included in imputation model } \\
\hline \multirow[t]{3}{*}{ MI-derPassive } & INT-within & $0.198(0.009)$ & $0.283(0.006)$ & $0.291(0)$ & $0.119(0.005)$ & 1.34 & $1(0)$ \\
\hline & INT-across & $-0.059(0.012)$ & $0.392(0.009)$ & $0.339(0.001)$ & $0.157(0.008)$ & 1.768 & $1(0)$ \\
\hline & INT-across2 & $-2.139(0.02)$ & $0.617(0.014)$ & $0.609(0.002)$ & $4.956(0.085)$ & 55.824 & $0(0)$ \\
\hline \multirow[t]{2}{*}{ MI-regActive } & INT-within & $0.241(0.01)$ & $0.316(0.007)$ & $0.31(0.001)$ & $0.158(0.007)$ & 1.78 & $1(0)$ \\
\hline & INT-across & $0.097(0.013)$ & $0.42(0.009)$ & $0.579(0.014)$ & $0.186(0.015)$ & 2.095 & $1(0)$ \\
\hline \multirow[t]{3}{*}{ MI-redActive } & INT-within & $0.251(0.01)$ & $0.316(0.007)$ & $0.31(0)$ & $0.163(0.007)$ & 1.836 & $1(0)$ \\
\hline & INT-across & $-0.062(0.014)$ & $0.433(0.01)$ & $0.406(0.003)$ & $0.191(0.011)$ & 2.151 & $1(0)$ \\
\hline & INT-across2 & $-2.082(0.02)$ & $0.617(0.014)$ & $0.616(0.002)$ & $4.715(0.082)$ & 53.11 & $0(0)$ \\
\hline
\end{tabular}

\section{MAR2A}

Auxiliary variable not included in imputation model

\begin{tabular}{|c|c|c|c|c|c|c|c|}
\hline \multirow[t]{3}{*}{ Ml-derPassive } & INT-within & $1.356(0.012)$ & $0.393(0.009)$ & $0.367(0.001)$ & $1.994(0.037)$ & 22.46 & $0(0)$ \\
\hline & INT-across & $1.248(0.018)$ & $0.559(0.013)$ & $0.567(0.005)$ & $1.87(0.058)$ & 21.064 & $0.352(0.015)$ \\
\hline & INT-across2 & $-0.801(0.017)$ & $0.526(0.012)$ & $0.511(0.001)$ & $0.918(0.029)$ & 10.34 & $0.994(0.002)$ \\
\hline \multirow[t]{2}{*}{ MI-regActive } & INT-within & $2.427(0.012)$ & $0.366(0.008)$ & $0.351(0)$ & $6.025(0.058)$ & 67.866 & $0(0)$ \\
\hline & INT-across & $3.362(0.049)$ & $1.559(0.035)$ & $1.408(0.005)$ & $13.732(0.358)$ & 154.677 & $0.035(0.006)$ \\
\hline \multirow[t]{3}{*}{ MI-redActive } & INT-within & $2.426(0.012)$ & $0.365(0.008)$ & $0.351(0)$ & $6.017(0.058)$ & 67.775 & $0(0)$ \\
\hline & INT-across & $4.829(0.024)$ & $0.757(0.017)$ & $0.873(0.011)$ & $23.891(0.233)$ & 269.108 & $0(0)$ \\
\hline & INT-across2 & $-0.705(0.017)$ & $0.529(0.012)$ & $0.523(0.001)$ & $0.776(0.027)$ & 8.741 & $1(0)$ \\
\hline \multicolumn{8}{|c|}{ Auxiliary variable included in imputation model } \\
\hline \multirow[t]{3}{*}{ MI-derPassive } & INT-within & $0.232(0.009)$ & $0.293(0.007)$ & $0.289(0)$ & $0.139(0.006)$ & 1.566 & $1(0)$ \\
\hline & INT-across & $-0.018(0.011)$ & $0.341(0.008)$ & $0.327(0.001)$ & $0.116(0.005)$ & 1.307 & $1(0)$ \\
\hline & INT-across2 & $-0.886(0.017)$ & $0.543(0.012)$ & $0.524(0.001)$ & $1.08(0.032)$ & 12.165 & $0.979(0.005)$ \\
\hline MI-regActive & INT-within & $0.525(0.01)$ & $0.327(0.007)$ & $0.327(0)$ & $0.382(0.014)$ & 4.303 & $1(0)$ \\
\hline
\end{tabular}




\section{MULTIPLE IMPUTATION IN PROPENSITY SCORE MATCHING}

\begin{tabular}{|c|c|c|c|c|c|c|c|}
\hline & INT-across & $0.731(0.052)$ & $1.644(0.037)$ & $1.322(0.008)$ & $3.235(0.154)$ & 36.439 & $1(0)$ \\
\hline \multirow[t]{3}{*}{ MI-redActive } & INT-within & $0.524(0.01)$ & $0.327(0.007)$ & $0.327(0)$ & $0.381(0.014)$ & 4.292 & $1(0)$ \\
\hline & INT-across & $-0.077(0.012)$ & $0.385(0.009)$ & $0.435(0.005)$ & $0.154(0.008)$ & 1.735 & $1(0)$ \\
\hline & INT-across2 & $-0.815(0.017)$ & $0.526(0.012)$ & $0.512(0.001)$ & $0.94(0.029)$ & 10.588 & $0.999(0.001)$ \\
\hline
\end{tabular}

MNAR

Auxiliary variable not included in imputation model

\begin{tabular}{|c|c|c|c|c|c|c|c|}
\hline \multirow[t]{3}{*}{ MI-derPassive } & INT-within & $1.369(0.013)$ & $0.404(0.009)$ & $0.368(0.001)$ & $2.038(0.038)$ & 22.956 & $0(0)$ \\
\hline & INT-across & $1.266(0.019)$ & $0.598(0.013)$ & $0.571(0.005)$ & $1.96(0.067)$ & 22.077 & $0.344(0.015)$ \\
\hline & INT-across2 & $-0.96(0.016)$ & $0.498(0.011)$ & $0.511(0.001)$ & $1.17(0.031)$ & 13.179 & $0.682(0.015)$ \\
\hline \multirow[t]{2}{*}{ MI-regActive } & INT-within & $2.47(0.012)$ & $0.365(0.008)$ & $0.353(0)$ & $6.236(0.059)$ & 70.242 & $0(0)$ \\
\hline & INT-across & $3.146(0.047)$ & $1.492(0.033)$ & $1.408(0.005)$ & $12.123(0.321)$ & 136.553 & $0.119(0.01)$ \\
\hline \multirow[t]{3}{*}{ Ml-redActive } & INT-within & $2.46(0.012)$ & $0.368(0.008)$ & $0.353(0)$ & $6.189(0.059)$ & 69.713 & $0(0)$ \\
\hline & INT-across & $4.852(0.024)$ & $0.763(0.017)$ & $0.84(0.009)$ & $24.124(0.232)$ & 271.732 & $0(0)$ \\
\hline & INT-across2 & $-0.801(0.017)$ & $0.529(0.012)$ & $0.525(0.001)$ & $0.922(0.029)$ & 10.385 & $1(0)$ \\
\hline \multicolumn{8}{|c|}{ Auxiliary variable included in imputation model } \\
\hline \multirow[t]{3}{*}{ MI-derPassive } & INT-within & $0.259(0.009)$ & $0.282(0.006)$ & $0.289(0)$ & $0.147(0.006)$ & 1.656 & $1(0)$ \\
\hline & INT-across & $-0.014(0.011)$ & $0.36(0.008)$ & $0.328(0.001)$ & $0.129(0.007)$ & 1.453 & $1(0)$ \\
\hline & INT-across2 & $-1.044(0.016)$ & $0.516(0.012)$ & $0.522(0.001)$ & $1.356(0.036)$ & 15.274 & $0.367(0.015)$ \\
\hline \multirow[t]{2}{*}{ MI-regActive } & INT-within & $0.557(0.01)$ & $0.317(0.007)$ & $0.329(0.001)$ & $0.411(0.013)$ & 4.629 & $1(0)$ \\
\hline & INT-across & $0.942(0.053)$ & $1.68(0.038)$ & $1.367(0.008)$ & $3.707(0.163)$ & 41.756 & $1(0)$ \\
\hline \multirow[t]{3}{*}{ Ml-redActive } & INT-within & $0.559(0.01)$ & $0.316(0.007)$ & $0.328(0.001)$ & $0.412(0.013)$ & 4.641 & $0.999(0.001)$ \\
\hline & INT-across & $-0.103(0.013$ & $0.416(0.009)$ & $0.449(0.005)$ & $0.184(0.011)$ & 2.073 & $1(0)$ \\
\hline & INT-across2 & $-0.967(0.016)$ & $0.5(0.011)$ & $0.509(0.001)$ & $1.184(0.032)$ & 13.337 & $0.672(0.015)$ \\
\hline
\end{tabular}




\section{LING ET AL.}

Table A11. Sensitivity simulation results: bias, standard error, mean squared error (MSE), and coverage (using bootstrap standard error) results using MI-derPassive INT-within strategy (auxiliary variable was not included in the imputation model) when varying the number of subjects, missing rate, or number of multiply imputed datasets compared to main simulation study (Monte Carlo standard errors in parentheses). (MDM = missing data mechanism, $m$ indicates the number of multiply imputed datasets). The results from the main simulations were highlighted in grey as reference.

\begin{tabular}{|c|c|c|c|c|c|c|c|c|}
\hline \multirow[b]{2}{*}{ MDM } & \multirow[b]{2}{*}{$\mathbf{m}$} & \multirow{2}{*}{$\begin{array}{l}\text { Missing } \\
\text { Rate (\%) }\end{array}$} & \multirow{2}{*}{$\begin{array}{c}\text { Number } \\
\text { of } \\
\text { Subjects }\end{array}$} & \multirow[b]{2}{*}{ Bias } & \multicolumn{2}{|c|}{ Standard Error } & \multirow[b]{2}{*}{ MSE } & \multirow[b]{2}{*}{ Coverage } \\
\hline & & & & & Empirical & Bootstrap & & \\
\hline \multicolumn{9}{|c|}{ Varying the number of subjects } \\
\hline MCAR & 50 & 50 & 2000 & $-0.001(0.01)$ & $0.316(0.007)$ & $0.315(0)$ & $0.099(0.004)$ & $1(0)$ \\
\hline MCAR & 50 & 50 & 1000 & $-0.005(0.122)$ & $0.545(0.012)$ & $0.557(0.001)$ & $0.297(0.095)$ & $1(0)$ \\
\hline MCAR & 50 & 50 & 500 & $-0.005(0.173)$ & $0.772(0.017)$ & $0.786(0.002)$ & $0.596(0.19)$ & $1(0)$ \\
\hline MCAR & 50 & 50 & 250 & $0.042(0.253)$ & $1.131(0.025)$ & $1.087(0.004)$ & $1.282(0.417)$ & $1(0)$ \\
\hline MAR1 & 50 & 50 & 2000 & $0.038(0.014)$ & $0.454(0.01)$ & $0.446(0.001)$ & $0.207(0.011)$ & $1(0)$ \\
\hline MAR1 & 50 & 50 & 1000 & $0.11(0.14)$ & $0.626(0.014)$ & $0.63(0.002)$ & $0.404(0.135)$ & $1(0)$ \\
\hline MAR1 & 50 & 50 & 500 & $0.22(0.192)$ & $0.859(0.019)$ & $0.878(0.005)$ & $0.786(0.255)$ & $1(0)$ \\
\hline MAR1 & 50 & 50 & 250 & $0.374(0.289)$ & $1.294(0.029)$ & $1.138(0.005)$ & $1.814(0.653)$ & $1(0)$ \\
\hline MAR2A & 50 & 50 & 2000 & $1.356(0.012)$ & $0.393(0.009)$ & $0.367(0.001)$ & $1.994(0.037)$ & $0(0)$ \\
\hline MAR2A & 50 & 50 & 1000 & $1.478(0.148)$ & $0.659(0.015)$ & $0.676(0.004)$ & $2.619(0.469)$ & $1(0)$ \\
\hline MAR2A & 50 & 50 & 500 & $1.516(0.208)$ & $0.931(0.021)$ & $0.939(0.007)$ & $3.165(0.757)$ & $0(0)$ \\
\hline MAR2A & 50 & 50 & 250 & $1.624(0.303)$ & $1.353(0.03)$ & $1.195(0.007)$ & $4.466(1.284)$ & $0(0)$ \\
\hline MNAR & 50 & 50 & 2000 & $1.369(0.013)$ & $0.404(0.009)$ & $0.368(0.001)$ & $2.038(0.038)$ & $0(0)$ \\
\hline MNAR & 50 & 50 & 1000 & $1.471(0.146)$ & $0.653(0.015)$ & $0.675(0.004)$ & $2.591(0.459)$ & $0(0)$ \\
\hline MNAR & 50 & 50 & 500 & $1.531(0.209)$ & $0.933(0.021)$ & $0.947(0.007)$ & $3.214(0.784)$ & $0.995(0.002)$ \\
\hline MNAR & 50 & 50 & 250 & $1.651(0.303)$ & $1.354(0.03)$ & $1.214(0.007)$ & $4.56(1.295)$ & $0(0)$ \\
\hline \multicolumn{9}{|c|}{ Varying missing rate } \\
\hline MCAR & 50 & 50 & 2000 & $-0.001(0.01)$ & $0.316(0.007)$ & $0.315(0)$ & $0.099(0.004)$ & $1(0)$ \\
\hline MCAR & 25 & 25 & 2000 & $0.006(0.057)$ & $0.361(0.008)$ & $0.37(0.001)$ & $0.13(0.029)$ & $1(0)$ \\
\hline MCAR & 10 & 10 & 2000 & $0.002(0.036)$ & $0.361(0.008)$ & $0.363(0.001)$ & $0.13(0.018)$ & $1(0)$ \\
\hline MAR1 & 50 & 50 & 2000 & $0.038(0.014)$ & $0.454(0.01)$ & $0.446(0.001)$ & $0.207(0.011)$ & $1(0)$ \\
\hline
\end{tabular}




\section{MULTIPLE IMPUTATION IN PROPENSITY SCORE MATCHING}

\begin{tabular}{|c|c|c|c|c|c|c|c|c|}
\hline MAR1 & 25 & 25 & 2000 & $0.021(0.062)$ & $0.39(0.009)$ & $0.389(0.001)$ & $0.153(0.035)$ & $1(0)$ \\
\hline MAR1 & 10 & 10 & 2000 & $0.005(0.036)$ & $0.362(0.008)$ & $0.365(0.001)$ & $0.131(0.019)$ & $1(0)$ \\
\hline MAR2A & 50 & 50 & 2000 & $1.356(0.012)$ & $0.393(0.009)$ & $0.367(0.001)$ & $1.994(0.037)$ & $0(0)$ \\
\hline MAR2A & 25 & 25 & 2000 & $0.779(0.061)$ & $0.383(0.009)$ & $0.379(0.001)$ & $0.753(0.101)$ & $0.05(0.007)$ \\
\hline MAR2A & 10 & 10 & 2000 & $0.202(0.035)$ & $0.355(0.008)$ & $0.358(0.001)$ & $0.167(0.023)$ & $1(0)$ \\
\hline MNAR & 50 & 50 & 2000 & $1.369(0.013)$ & $0.404(0.009)$ & $0.368(0.001)$ & $2.038(0.038)$ & $0(0)$ \\
\hline MNAR & 25 & 25 & 2000 & $0.759(0.06)$ & $0.379(0.008)$ & $0.378(0.001)$ & $0.721(0.097)$ & $0.066(0.008)$ \\
\hline MNAR & 10 & 10 & 2000 & $0.198(0.035)$ & $0.352(0.008)$ & $0.358(0.001)$ & $0.163(0.023)$ & $1(0)$ \\
\hline \multicolumn{9}{|c|}{ Varying the number of multiply imputed dataset, $m$} \\
\hline MCAR & 50 & 50 & 2000 & $-0.001(0.01)$ & $0.316(0.007)$ & $0.315(0)$ & $0.099(0.004)$ & $1(0)$ \\
\hline MCAR & 10 & 50 & 2000 & $0.032(0.039)$ & $0.391(0.009)$ & $0.399(0.001)$ & $0.154(0.022)$ & $1(0)$ \\
\hline MAR1 & 50 & 50 & 2000 & $0.038(0.014)$ & $0.454(0.01)$ & $0.446(0.001)$ & $0.207(0.011)$ & $1(0)$ \\
\hline MAR1 & 10 & 50 & 2000 & $0.094(0.046)$ & $0.461(0.01)$ & $0.454(0.001)$ & $0.221(0.034)$ & $1(0)$ \\
\hline MAR2A & 50 & 50 & 2000 & $1.356(0.012)$ & $0.393(0.009)$ & $0.367(0.001)$ & $1.994(0.037)$ & $0(0)$ \\
\hline MAR2A & 10 & 50 & 2000 & $1.495(0.048)$ & $0.482(0.011)$ & $0.481(0.002)$ & $2.467(0.152)$ & $0(0)$ \\
\hline MNAR & 50 & 50 & 2000 & $1.369(0.013)$ & $0.404(0.009)$ & $0.368(0.001)$ & $2.038(0.038)$ & $0(0)$ \\
\hline MNAR & 10 & 50 & 2000 & $1.49(0.048)$ & $0.48(0.011)$ & $0.481(0.002)$ & $2.449(0.151)$ & $0.811(0.012)$ \\
\hline
\end{tabular}

